University of Tennessee Health Science Center

UTHSC Digital Commons

\title{
Characterization of the Sigma Factor Proteins and the Dna Binding Protein Euo of Chlamydia
}

\author{
Cory L. Blackwell \\ University of Tennessee Health Science Center
}

Follow this and additional works at: https://dc.uthsc.edu/dissertations

Part of the Female Urogenital Diseases and Pregnancy Complications Commons, Male Urogenital Diseases Commons, Medical Microbiology Commons, and the Medical Molecular Biology Commons

\section{Recommended Citation}

Blackwell, Cory L. , "Characterization of the Sigma Factor Proteins and the Dna Binding Protein Euo of Chlamydia" (2011). Theses and Dissertations (ETD). Paper 25. http://dx.doi.org/10.21007/ etd.cghs.2011.0028.

This Dissertation is brought to you for free and open access by the College of Graduate Health Sciences at UTHSC Digital Commons. It has been accepted for inclusion in Theses and Dissertations (ETD) by an authorized administrator of UTHSC Digital Commons. For more information, please contact jwelch30@uthsc.edu. 


\title{
Characterization of the Sigma Factor Proteins and the Dna Binding Protein Euo of Chlamydia
}

\begin{abstract}
Chlamydia spp. are prokaryotic obligate intracellular pathogens with a unique, biphasic developmental cycle in which an infectious, extracellular form termed the elementary body (EB) interconverts with a metabolically active intracellular reticulate body (RB) within host cells. Subsets of genes are differentially expressed during the developmental cycle, and these genes are believed to be responsible for the transitions between the EB and RB forms. The goal of these studies was to explore two potential mechanisms that may function in regulating developmental cycle stage-specific gene expression in chlamydiae: a cascade of sigma factor expression and the binding of the early stage protein EUO to DNA.

The protein expression levels of each of the three sigma factors were monitored throughout the developmental cycle to determine if expression of the sigma factors is consistent with a role in directing temporal gene regulation via a cascade mechanism. Western blot and immunofluorescence assays revealed that the sigma 66 , the major sigma factor, is present throughout the developmental cycle and, consequently, could direct temporal gene expression, but not via a simple cascade mechanism. The alternative sigma 28 protein was found to be highest during the late logarithmic phase, and it can be speculated that it plays a key role in the initial stages of RB-to-EB conversion through its regulation of the late DNA binding protein, histone HctB. Analysis of sigma 54 protein suggested it may exist in two forms: a cytoplasmic form, which most likely functions as a sigma factor during RB replication, and an outer membrane complex form, which is present in RBs and EBs and may have a structural function. Expression of the proposed functional sigma 54 protein throughout the logarithmic phase suggests that it plays no direct role in EB-RB differentiation via a cascade mechanism.
\end{abstract}

EUO is a DNA-binding protein that is produced early in the developmental cycle but is also present during the logarithmic phase of growth. Because transcription studies have suggested that euo is the most actively expressed gene within the first few hours of infection, EUO is believed to be a potential transcriptional regulator. A ChIP-to-Chip protocol was performed with isolated genomic DNA and recombinant EUO to identify genes to which EUO may bind. DNA that co-immunoprecipitated with the recombinant EUO was allowed to hybridize to a microarray that contained the intergenic regions and the open reading frames of the Chlamydia trachomatis serovar D genome. EUO binding was observed to be scattered throughout the genome, although with reproducible affinity for particular DNA fragments. However, direct evidence for regulation of key stage-specific genes was not obtained by this study.

An immunoprecipitation-cloning protocol was developed to identify in vivo binding sites of EUO. Sixteen hour post-infection RBs were formaldehyde cross-linked to preserve the physiological interactions between native EUO and DNA. EUO was immunoprecipitated from these cross-linked reticulate bodies and the EUO-bound DNA was cloned and sequenced. BLAST searches of these sequences identified thirty genes that may be in vivo targets of EUO, but the role of these genes in EB-RB interconversion is unclear.

Document Type

Dissertation

Degree Name

Doctor of Philosophy (PhD)

Program

Microbiology 
Research Advisor

Thomas P. Hatch, Ph.D.

Keywords

Chlamydia, EUO protein, Sigma factors, Transcription

\section{Subject Categories}

Diseases | Female Urogenital Diseases and Pregnancy Complications | Male Urogenital Diseases |

Medical Microbiology | Medical Molecular Biology | Medicine and Health Sciences 


\title{
CHARACTERIZATION OF THE SIGMA FACTOR PROTEINS AND THE DNA-BINDING PROTEIN EUO OF CHLAMYDIA
}

\author{
A Dissertation \\ Presented for \\ The Graduate Studies Council \\ The University of Tennessee \\ Health Science Center
}

\author{
In Partial Fulfillment \\ Of the Requirements for the Degree \\ Doctor of Philosophy \\ From The University of Tennessee
}

By

Cory L. Blackwell

May 2011 
Copyright (C) 2011 by Cory L. Blackwell. All rights reserved. 


\section{DEDICATION}

This work is dedicated to the memory of my father, Tom Blackwell, who instilled in me the ideas to never give up and to strive to be the best at all I do. To my grandmother, Julia Fairley, who always provided me with kind and uplifting words to help me through any situation.

This work is also dedicated to my mother, Joyce A. McDonald and my brother, Thomas D. Blackwell. You two were always there for me. For that I thank you. 


\section{ACKNOWLEDGEMENTS}

I would like to thank my faculty advisor, Thomas P. Hatch, for the continued patience and support throughout my graduate school career. I also would like to thank my committee members Dr. Robert J. Belland, Dr. James Bina, Dr. David Hasty, and Dr. Mark Miller for the additional guidance and support.

I would also like to thank the friends that I have met here during my graduate school tenure. Not only from an academic standpoint, providing me with ideas and suggestions for my project, but as well from a personal standpoint. I learned that one must have a balance in life and I thank them for helping provide that balance during my stay in Memphis. A special thanks goes to Shawn Clinton, Erika Dillard, and Timothy Sullivan.

Most importantly I have to acknowledge my family. Their continued support was pivotal to my matriculation as a graduate student. Ranging from missed holidays to unanswered phone calls, you always understood. The sacrifices you made were just as important as my own sacrifices. Our sacrifices were not and shall not be in vain. 


\begin{abstract}
Chlamydia spp. are prokaryotic obligate intracellular pathogens with a unique, biphasic developmental cycle in which an infectious, extracellular form termed the elementary body (EB) interconverts with a metabolically active intracellular reticulate body (RB) within host cells. Subsets of genes are differentially expressed during the developmental cycle, and these genes are believed to be responsible for the transitions between the EB and RB forms. The goal of these studies was to explore two potential mechanisms that may function in regulating developmental cycle stage-specific gene expression in chlamydiae: a cascade of sigma factor expression and the binding of the early stage protein EUO to DNA.
\end{abstract}

The protein expression levels of each of the three sigma factors were monitored throughout the developmental cycle to determine if expression of the sigma factors is consistent with a role in directing temporal gene regulation via a cascade mechanism. Western blot and immunofluorescence assays revealed that the sigma 66, the major sigma factor, is present throughout the developmental cycle and, consequently, could direct temporal gene expression, but not via a simple cascade mechanism. The alternative sigma 28 protein was found to be highest during the late logarithmic phase, and it can be speculated that it plays a key role in the initial stages of RB-to-EB conversion through its regulation of the late DNA binding protein, histone HctB. Analysis of sigma 54 protein suggested it may exist in two forms: a cytoplasmic form, which most likely functions as a sigma factor during RB replication, and an outer membrane complex form, which is present in RBs and EBs and may have a structural function. Expression of the proposed functional sigma 54 protein throughout the logarithmic phase suggests that it plays no direct role in EB-RB differentiation via a cascade mechanism.

EUO is a DNA-binding protein that is produced early in the developmental cycle but is also present during the logarithmic phase of growth. Because transcription studies have suggested that euo is the most actively expressed gene within the first few hours of infection, EUO is believed to be a potential transcriptional regulator. A ChIP-to-Chip protocol was performed with isolated genomic DNA and recombinant EUO to identify genes to which EUO may bind. DNA that co-immunoprecipitated with the recombinant EUO was allowed to hybridize to a microarray that contained the intergenic regions and the open reading frames of the Chlamydia trachomatis serovar D genome. EUO binding was observed to be scattered throughout the genome, although with reproducible affinity for particular DNA fragments. However, direct evidence for regulation of key stage-specific genes was not obtained by this study.

An immunoprecipitation-cloning protocol was developed to identify in vivo binding sites of EUO. Sixteen hour post-infection RBs were formaldehyde cross-linked to preserve the physiological interactions between native EUO and DNA. EUO was immunoprecipitated from these cross-linked reticulate bodies and the EUO-bound DNA was cloned and sequenced. BLAST searches of these sequences identified thirty genes 
that may be in vivo targets of EUO, but the role of these genes in EB-RB interconversion is unclear. 


\section{TABLE OF CONTENTS}

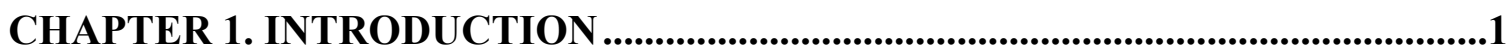

CHLAMYDIA NOMENCLATURE AND DISEASE ............................................

DEVELOPMENTAL CYCLE .........................................................................

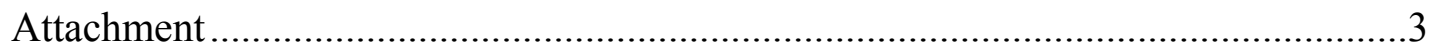

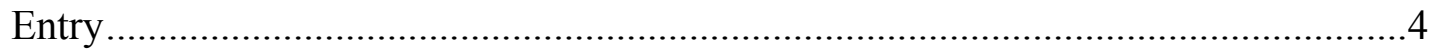

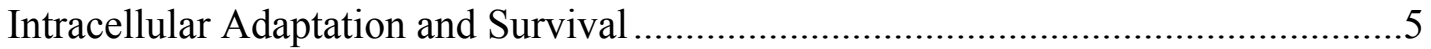

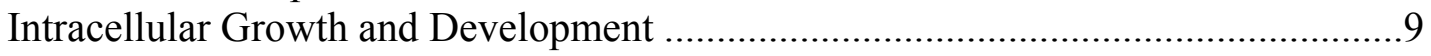

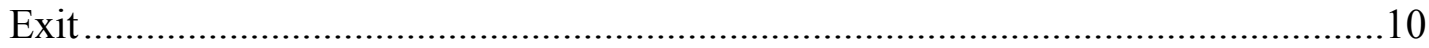

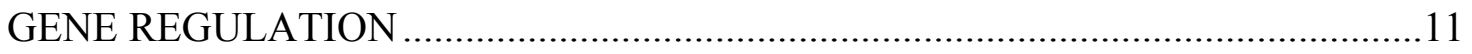

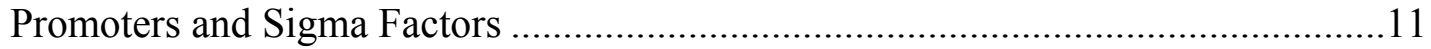

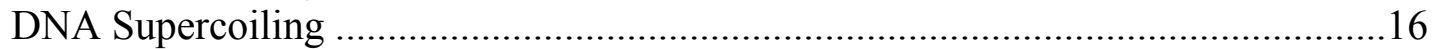

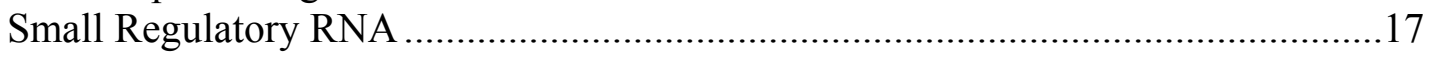

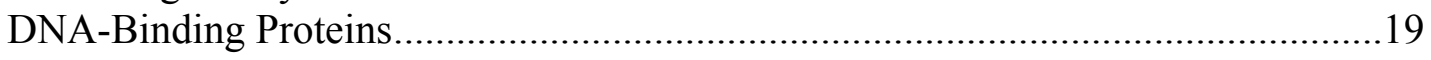

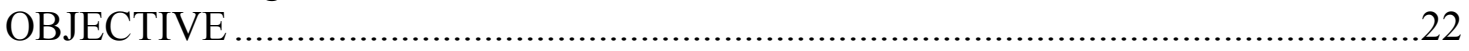

CHAPTER 2. PROTEIN EXPRESSION LEVELS OF SIGMA 66, SIGMA 54, AND SIGMA 28 THROUGHOUT THE DEVELOPMENTAL CYCLE OF

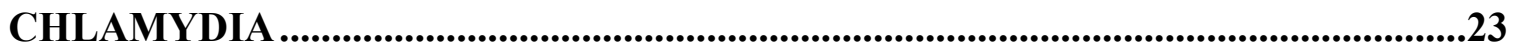

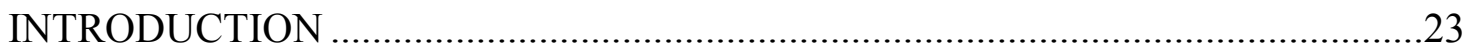

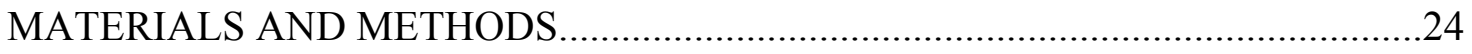

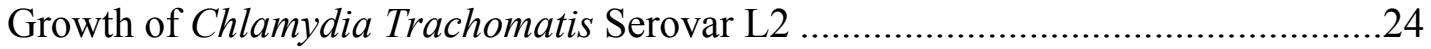

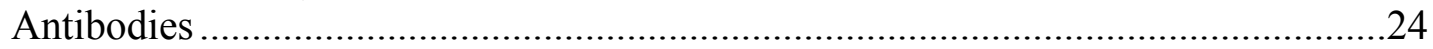

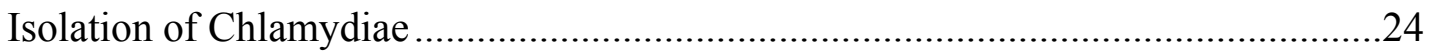

Sodium Dodecyl Sulfate-Polyacrylamide Gel Electrophoresis and Western Blot

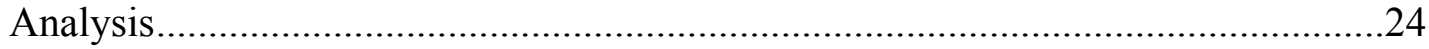

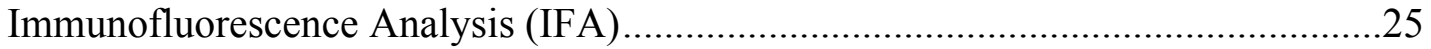

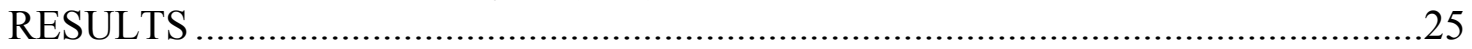

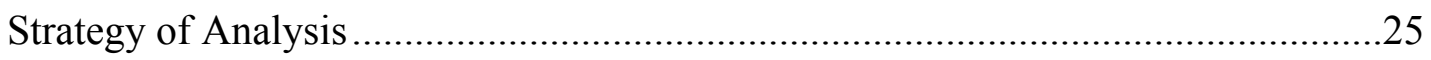

Sigma 66 Is Expressed throughout Developmental Cycle.....................................27

Sigma 54 Is Expressed throughout the Developmental Cycle of Chlamydia ............27

Sigma 28 Is Expressed during the Logarithmic and Early Late Phases of

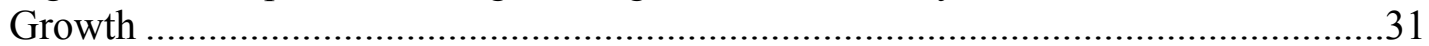

Polyclonal Sigma 54 Cross-Reacts with an Antigen in the OMC .............................31

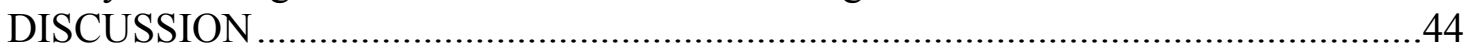

\section{CHAPTER 3. IN VITRO IDENTIFICATION OF EUO BINDING SITES} USING CHROMATIN IMMUNOPRECIPITATION TO MICROARRAY

CHIP ANALYSIS .......................................................................................48

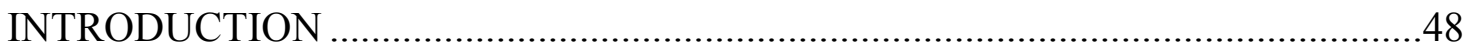

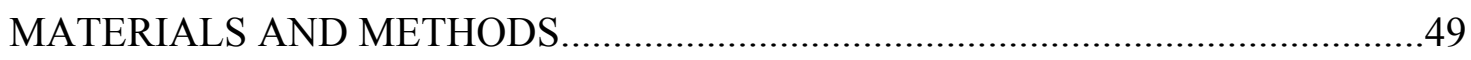

Growth and Isolation of Chlamydia Trachomatis Serovar D ................................49

Preparation of Recombinant His-Tagged EUO ..................................................49

Electrophoresis Mobility Shift Assay .............................................................49 
Immunoprecipitation of EUO from RB and Recombinant EUO/Co-

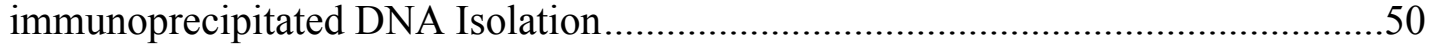

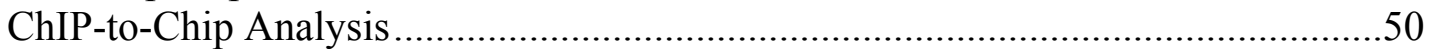

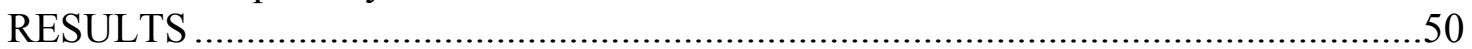

Identification of EUO in Chlamydia Trachomatis Serovar D ..................................50

Chlamydia Trachomatis Serovar D Binds DNA ................................................51

Immunoprecipitation of EUO from C. Trachomatis Serovar D Extracts ..................51

Isolation of Co-Immunoprecipitated DNA from EUO Immunoprecipitations...........55

Immunoprecipitation of Recombinant His-Tagged EUO and Isolation of Co-

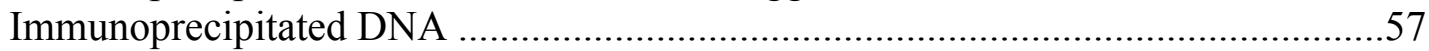

EUO Binds Multiple Regions throughout the Chlamydial Genome .......................60

EUO Does Not Preferentially Bind AT-Rich Sequences......................................60

Analysis of Selected Intergenic Regions and Open Reading Frames .......................64

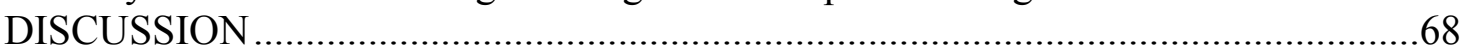

\section{CHAPTER 4. IN VIVO IDENTIFICATION OF EUO BINDING SITES USING} CHROMATIN IMMUNOPRECIPITATION AND CLONING ANALYSIS...........70

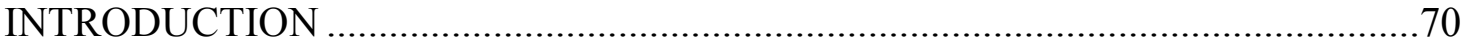

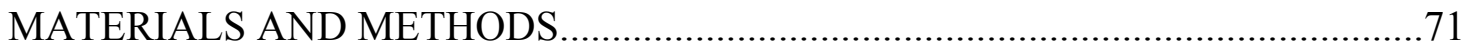

Growth and Isolation of Chlamydia Psittaci ............................................................ 71

Immunoprecipitation of EUO from Infected Cells ............................................... 71

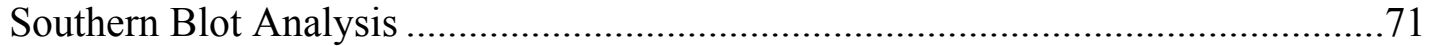

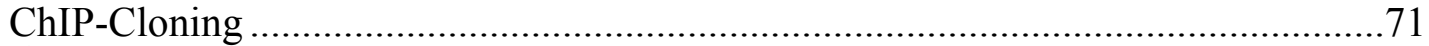

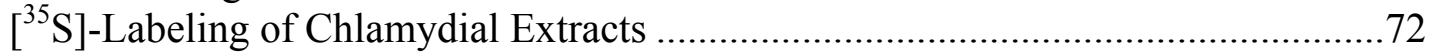

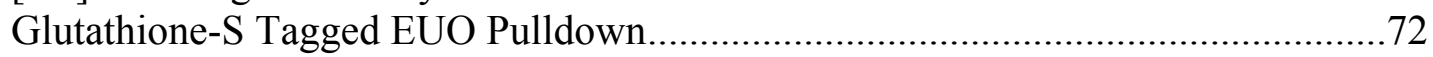

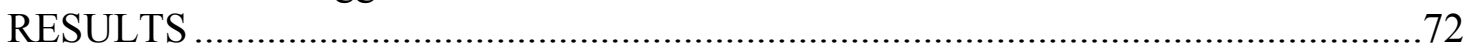

Identification of EUO from Chlamydia Psittaci Extracts .....................................72

Immunoprecipitation of EUO from Chlamydia Psittaci Extracts............................72

ChIP-Cloning Identifies Several In Vivo Targets of EUO .................................. 74

Immunoprecipitation of EUO from Infected Cells ............................................79

Isolation and Sequencing of EUO Co-Immunoprecipitated DNA from

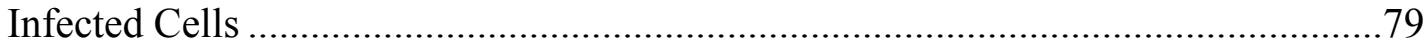

DNase Contains Proteolytic Activity ................................................................. 79

Binding of Proteins to EUO Was Not Detected via Co-Immunoprecipitations

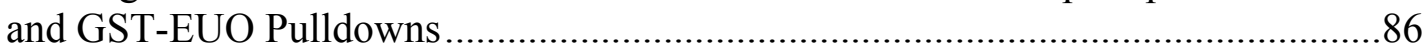

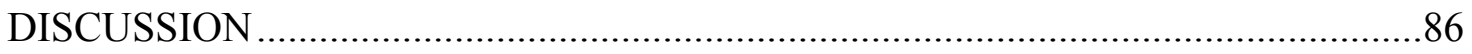

CHAPTER 5. GENERAL DISCUSSION ..............................................................

SIGMA FACTORS ROLE IN TRANSCRIPTIONAL REGULATION IN

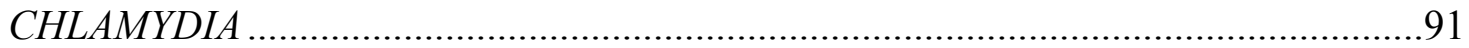

Expression of the Sigma Factor Proteins ................................................................. 91

Detection of an Unknown Antigen with Sigma 54 Polyclonal Serum .....................92

BINDING STUDIES OF THE DNA-BINDING PROTEIN EUO …......................93

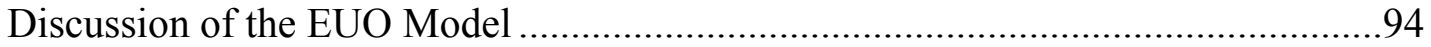

EUO Protein Interaction with Other Proteins and Its Degradation..........................94

In Vitro Binding Specificity of EUO ............................................................ 96 


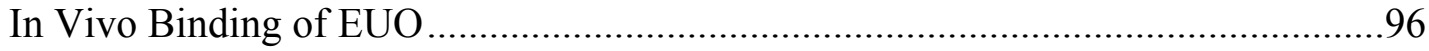

EUO AS A POSSIBLE RNA CHAPERONE .....................................................97

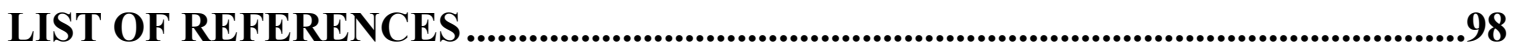

VITA 


\section{LIST OF TABLES}

Table 3-1. Probes with the strongest hybridization to EUO co-immunoprecipitated

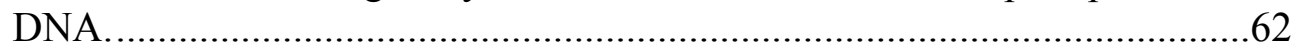

Table 3-2. Probes with the weakest hybridizations to EUO co-immunoprecipitated

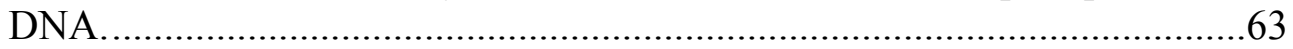

Table 3-3. Ranking of the ORFs with the highest hybridization values to EUO co-

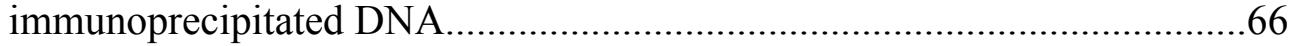

Table 3-4. Ranking of the ORFs with the lowest hybridization values to EUO coimmunoprecipitated DNA.............................................................67

Table 4-1. List of genes identified with BLAST search of EUO coimmunoprecipitated DNA. 


\section{LIST OF FIGURES}

Figure 2-1. Coomassie stain of reticulate bodies......................................................26

Figure 2-2. IFA of sigma 66 in C. trachomatis-infected cells.......................................28

Figure 2-3. Detection of sigma 66 protein in reticulate bodies. ......................................29

Figure 2-4. IFA of sigma 54 in C. trachomatis-infected cells........................................30

Figure 2-5. Detection of sigma 54 in RBs by western blot analysis.................................32

Figure 2-6. IFA of sigma 28 in C. trachomatis-infected cells.........................................33

Figure 2-7. Detection of sigma 28 in RB extracts by western blot...................................34

Figure 2-8. Polyclonal vs monoclonal IFA of sigma 54 in C. trachomatis-infected

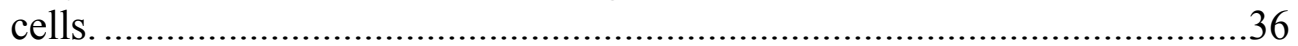

Figure 2-9. IFA of sigma 54 in C. trachomatis-infected cells during the developmental cycle using monoclonal antibody...........................................37

Figure 2-10. IFA of sigma 54 in purified C. trachomatis EBs........................................39

Figure 2-11. Western blot analysis of His-tagged sigma 54 protein. ..................................40

Figure 2-12. Western blot analysis of reticulate bodies using sigma 54 monoclonal antibodies and sigma 54 polyclonal serum.....

Figure 2-13. Immunofluorescence microscopy of C. trachomatis outer membrane complexes (OMCs).

Figure 2-14. Immunofluorescence microscopy of C. trachomatis EBs treated with trypsin.

Figure 2-15. Western blot detection of MOMP and sigma 54 in EBs.............................45

Figure 3-1. Detection of EUO in Chlamydia trachomatis serovar D extracts..................52

Figure 3-2. Cysteine-rich protein (CRP) operon. ............................................................53

Figure 3-3. Electrophoretic mobility shift assay of the cysteine-rich protein operon

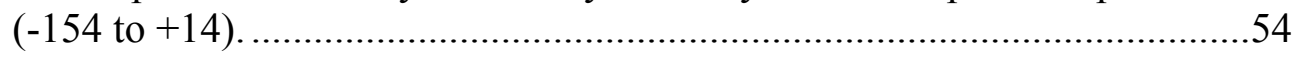

Figure 3-4. Immunoprecipitation of EUO from Chlamydia trachomatis serovar D

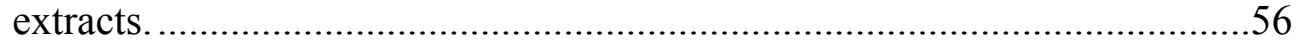

Figure 3-5. Immunoprecipitation of recombinant EUO............................................58 
Figure 3-6. Isolation and amplification of EUO co-immunoprecipitated DNA....

Figure 3-7. ChIP-to-Chip layout of EUO co-immunoprecipitated DNA hybridized to the $C$. trachomatis serovar D genome.

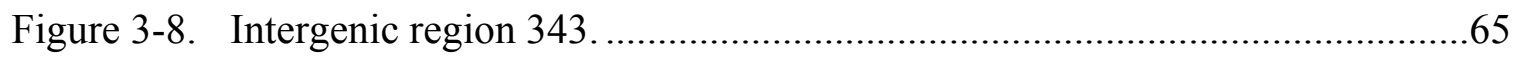

Figure 4-1. Detection of EUO in Chlamydia psittaci extracts.....................................73

Figure 4-2. Immunoprecipitation of EUO from formaldehyde cross-linked Chlamydia psittaci RBs.

Figure 4-3. Amplification of EUO co-immunoprecipitated DNA...............................76

Figure 4-4. Southern blot analysis of EUO co-immunoprecipitated DNA. ...................77

Figure 4-5. Immunoprecipitation of EUO from cross-linked infected cells at 2 hpi.......80

Figure 4-6. Immunoprecipitation of EUO from cross-linked infected cells at 3 hpi.......81

Figure 4-7. Immunoprecipitation of EUO from cross-linked infected cells at 4 hpi.......82

Figure 4-8. Amplification of EUO co-immunoprecipitated DNA...............................83

Figure 4-9. Effect of DNase treatment on EUO immunoprecipitation............................84

Figure 4-10. Effect of DNase treatment of infected cell extracts on EUO......................85

Figure 4-11. Effect of DNase on chlamydial extracts and HSP-60 detection. .................87

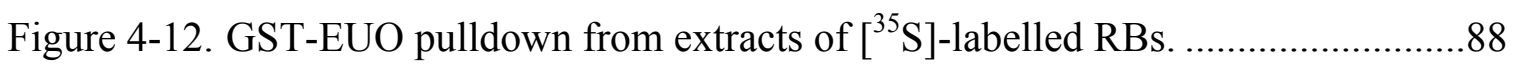

Figure 4-13. EUO immunoprecipitation from extracts of $\left[{ }^{35} \mathrm{~S}\right]$-labelled RBs..................89 


\section{CHAPTER 1. INTRODUCTION}

\section{CHLAMYDIA NOMENCLATURE AND DISEASE}

Species of the genus Chlamydia are prokaryotic obligate intracellular bacteria with a wide host tropism that cause an array of diseases in humans and animal, including the most common preventable sexually transmitted disease in the United States. The National Institute of Allergy and Infectious Diseases reports 4 to 8 million new cases Chlamydia trachomatis infections occur each year (1999). In the United States, the direct medical cost of chlamydial sexually transmitted disease was estimated to approach 30 million dollars annually, with the incidence of 5259 infections per 100,000 people in 2006 (Pultorak, Wong et al. 2009). The consequences of these chlamydial infections are estimated to indirectly cost the United States more than 2 billion dollars annually (Stamm 1999), and these figures do not take into account the population of men and women who are asymptomatic for the disease. Chlamydiae pose a major problem on a global stage as well. The World Health Organization (WHO) estimated that 92 million people were infected with chlamydiae around the world in 1999. C. trachomatis is the causative agent of trachoma, the leading cause of preventable blindness in the world, having the greatest impact on developing countries (Resnikoff, Pascolini et al. 2004). The World Health Organization has initiated a program in an attempt to eradicate blinding trachoma by the year 2020 (Negrel and Mariotti 1998).

The order Chlamydiales contains the families Chlamydiaceae, Parachlamydiaceae, Waddliaceae, and Simkaniaceae (Bush and Everett 2001). The family Chlamydiaceae is divided into two genera, Chlamydia and Chlamydophila based on phylogenetic analysis of the $16 \mathrm{~s}$ and $23 \mathrm{~s}$ rRNA. However, most investigators in the Chlamydia research field dispute the splitting of the genus (Stephens, Myers et al. 2009) and the single genus name Chlamydia is commonly accepted in publications. There are nine species in the Chlamydia genus: $C$. muridarum, $C$. trachomatis, Chlamydia suis, $C$. abortus, C. caviae, C. felis, C. pecorum, C. pneumoniae, and C. psittaci (Everett, Bush et al. 1999).

Three of the nine species of Chlamydia are known to cause disease in humans. $C$. psittaci is the causative agent of psittacosis. Although it is avian in nature, continuous exposure to infected birds or bird droppings can cause the transmission of the pathogen to a human host. Psittacosis can range from an inapparent sickness to severe pneumonia, endocarditis, and encephalitis. Although outbreaks of psittacosis have declined, some regions of the world are experiencing an increase in incidence rates (Andersen and Vanrompay 2000). The incubation time of $C$. psittaci in humans ranges between one to four weeks. The symptoms usually are present around day ten. Tetracycline and chloramphenicol are administered to patients with psittacosis. The Center for Disease Control has labeled C. psittaci as a category B biological weapons agent.

Chlamydia pneumoniae is a common cause of upper respiratory infections and pneumonia in humans. It is estimated to cause up to ten percent of the community- 
acquired pneumonia and five percent of bronchitis and sinusitis cases (Aldous, Grayston et al. 1992). It affects all age groups but is most common amongst the elderly and children between the ages of five and fourteen years of age (Grayston 1992). The Center for Disease Control estimates that there are two to five million cases of pneumonia annually resulting in half a million hospitalizations. Azithromycin or clarithromycin are the primary antibiotics administered for treatment of pneumonia caused by $C$.

pneumoniae. C. pneumoniae has also been shown to be associated with chronic coronary heart disease, acute myocardial infarction (Saikku, Leinonen et al. 1988) and asthma (Hahn, Bukstein et al. 1998).

Chlamydia trachomatis is the third species that commonly causes disease in humans. It is a major agent of ocular infections such as trachoma and infant inclusion conjunctivitis or can be the cause of sexually transmitted diseases such as urethritis, cervicitis, epididymitis, and pelvic inflammatory disease. C. trachomatis is divided into three different biovars: trachoma, lymphogranuloma venerum (LGV), and mouse pneumonitis. The biovars, trachoma and LGV, have been sub-classified into serovars A$\mathrm{K}$ and L1, L2, and L3, respectively (Wang, Kuo et al. 1985), with the addition of serovars $\mathrm{Da}$, Ia, and L2a in 1991 (Wang and Grayston 1991). Serovars A-C are responsible for the ocular infections such as trachoma. Serovars D-K are responsible for infections in the genital tract. The L serovars cause LGV, which is invasive and leads to the infection of the lymphatics and lymph nodes. As the organism travels through the lymphatic system, it multiplies in the mononuclear phagocytes of the lymph nodes. A breakout of LGV in the Netherlands in 2004 amongst men who have sex with men has led to a dissemination of the disease in Europe and the United States (Kivi, Koedijk et al. 2008).

\section{DEVELOPMENTAL CYCLE}

Chlamydiae are intracellular pathogens with a unique biphasic developmental cycle (Moulder 1982). The infectious, extracellular form of chlamydiae is known as the elementary body (EB). It is an electron-dense structure measuring 0.3 micrometers in diameter. EBs are osmotically stable, in part to the extensive cross-linking of their cell walls (Tamura, Matsumoto et al. 1971) and possess a condensed nucleoid structure which is compacted by histone-like proteins (Perara, Ganem et al. 1992). The EB also contains surface projections that are anchored to the cytoplasmic membrane (Matsumoto 1982). Once internalized inside the host cell, the EB differentiates to the replicative, metabolically active form known as the reticulate body (RB). The RB is larger in size than the EB, measuring one micrometer in diameter. The RB also lacks the extensive cross-linking of the outer membrane as seen within the EB (Hatch, Miceli et al. 1986). In this form, chlamydiae begin to grow and replicate via binary fission within a vacuole called an inclusion. Through an unknown mechanism, the inclusion evades fusion with lysosomes. After a period of growth and replication, a yet-to-be-established signal triggers the RBs to differentiate back into EBs; the EBs are then released from the cell and begin another round of infection. 


\section{Attachment}

The initial step of infection is the attachment of the EB to the outer surface of the host cell. The process of attachment has been widely studied, although a definitive mechanism has yet to be elucidated. Several factors, both of host and chlamydial origin, play a possible role in the attachment process. Kuo and Grayston observed in 1976 that the presence of heparin and heparin sulfate causes a loss in infectivity of $C$. trachomatis (1976). Heparin is a member of the glycosaminoglycan (GAG) family, which consists of long unbranched polysaccharides comprised of repeating disaccharide moieties. More recent analysis has also illustrated the importance of GAGs during a chlamydial infection. Zhang and Stephens showed that using purified GAGs and GAG lyases inhibits infection by chlamydiae (1992). The infectivity of chlamydiae in the presence of heparin is also biovar and serovar specific. The LGV biovar uses a heparin-inhibitable mechanism for attaching to host cells, whereas the trachoma biovar uses a heparinindependent mechanism (Chen and Stephens 1997). A separate study comparing the affects of GAGs on the infection rates of $C$. trachomatis serovars LGV and E showed that serovar LGV and not serovar $\mathrm{E}$ is dependent on host cell heparin sulfate (Taraktchoglou, Pacey et al. 2001). In vitro studies have shown that heparin sulfate inhibits infection, and an in vivo study demonstrated the inability of several sulfated polysaccharides (heparin, dextran sulfate, and pentosate polysulfate) to provide a protective efficacy in a murine model of chlamydial genetic tract infection (Su and Caldwell 1998). It has been proposed that the inhibitory effects of heparin may be a non-specific charge-mediated interaction and that other, more specific interactions must be present to complete the attachment process.

Several chlamydial proteins have been identified as possible adhesion molecules that help the pathogen to attach to the host cell. In an attempt to define surface components that may be a part in the attachment process, Su et al. treated EBs with trypsin and monitored its effect on infectivity (1988). Trypsin proteolysis resulted in the cleavage of the major outer membrane protein (MOMP) and a loss in attachment and infectivity. It was also noted that the cleavage of MOMP by trypsin was serovar-specific. There were two cleavage sites in serovar B MOMP but only one site in serovar L2 MOMP. The additional cleavage site in serovar B MOMP was concluded to be the major determinant in the loss of infectivity because the single cleavage site of serovar L2 was insufficient in causing the loss of infectivity. Another protein that has been implicated as having a role in attachment is the cysteine-rich outer membrane complex B protein $(\mathrm{OmcB})$. OmcB elicits a strong antibody response, suggesting a surface localization (Wagels, Rasmussen et al. 1994). It is conserved among all Chlamydia spp., thereby serving as a possible candidate for being an adhesion molecule of chlamydiae. Using yeast-display technology where OmcB is presented on the surface of yeast, it was observed that these yeast cells were able to bind to human epithelial cells (Moelleken and Hegemann 2008). In a different study, infectivity infection assays were carried out using recombinant OmcB protein and a decrease in infectivity was seen in both LGV and B serovars (Fadel and Eley 2008). However, studies by the Hatch lab (Everett and Hatch 1995) suggest that OmcB is located in the periplasm and is not surface exposed. Failure to identify surface exposure has been confirmed by others (Watson, Lambden et al. 1994) 
and the role of $\mathrm{OmcB}$ in attachment remains controversial. Polymorphic membrane protein $\mathrm{D}(\mathrm{PmpD})$ is another highly conserved protein that has been implicated as a possible adhesion. Addition of antibodies raised against PmpD resulted in a decrease of infectivity of EBs (Wehrl, Brinkmann et al. 2004). Several other chlamydial proteins have been shown to play a role in the attachment process, but a definitive attachment mechanism has yet to be elucidated.

The attachment process of a pathogen not only depends on virulence factors from the pathogen but also is dependent on host cell determinants. Researchers have shown that the chlamydial attachment process is protein disulfide isomerase dependent (PDI) (Conant and Stephens 2007). In this study, Chinese Hamster Ovary 6 (CHO6) was used to monitor the attachment of $C$. trachomatis. $\mathrm{CHO6}$ cells are normally resistant to chlamydial attachment and entry due to $\mathrm{CHO} 6$ cells having a defect in processing the leader sequence for protein disulfide isomerase, causing it to be inactive. Complementation of the full-length protein disulfide isomerase resulted in the ability of C. trachomatis to attach and enter the host cell. The Wyrick lab (Davis, Raulston et al. 2002) also demonstrated that chlamydial attachment is dependent on the reduction of the EB cross-linked outer membranes. They treated human endometrial epithelial cells with PDI inhibitors at the time of EB attachment which resulted in reduced chlamydial infectivity. From the many studies performed and the various factors which have been shown to play a role in the attachment of chlamydia to the target cell, it would appear that attachment is a multi-step process.

\section{Entry}

Following attachment to the host cell, the EB is then taken up into the host cell via endocytosis. The details of the mechanism behind the uptake of EBs have been debated. Early studies suggested that the EB enters the target cell via clathrin-coated pits (Hodinka and Wyrick 1986). The Wyrick lab demonstrated that C. trachomatis entered polarized epithelial cells through clathrin-mediated endocytosis (Wyrick, Choong et al. 1989). Majeed et al. utilized immunofluorescence to monitor the distribution and localization of EBs, F-actin, and clathrin in cells infected with chlamydiae. It was observed that polymerized actin and clathrin played a role in the redistribution of chlamydiae into an intracellular aggregate (Majeed and Kihlstrom 1991). Some reports have observed chlamydia being endocytosed in vesicles void of clathrin (Murray and Ward 1984). Norkin et al. (2001) examined the possibility of EBs being endocytosed in a caveolae-mediated pathway and similarities were observed between the entry of chlamydiae and a caveolae-mediated pathway mediated by simian virus 40 (SV40). $C$. trachomatis serovar K was noted to enter three different cell types via caveolincontaining sphingholipid and cholesterol-enriched raft microdomains of the cell plasma membranes. C. trachomatis serovars E and F, C. psittaci, and C. pneumoniae were also seen to enter the cell in a similar fashion (Stuart, Webley et al. 2003). The Stuart et al. study also showed that $C$. trachomatis serovars A, C, L2, and mouse pneumonitis (MoPn) entered the cell via a caveolin-independent manner, raising the possibility that the method of entry may vary from one strain/species of Chlamydia to another. Further evidence of 
this is the association of the chlamydial inclusion with the host protein, calveolin-2. Calveolin-2 and not calveolin-1 was seen to associate with inclusions of $C$. trachomatis serovars A, B, and C (Webley, Norkin et al. 2004). A more recent study used RNA interference to disrupt the production of proteins involved in either clathrin-mediated, caveola-mediated, phagocytosis, and macropinocytosis mechanisms of endocytosis (Hybiske and Stephens 2007). The outcome of this study was that clathrin-mediated endocytosis was responsible for the entry of chlamydial EBs, although other additional mechanisms may also have a role in uptake.

One of the additional mechanisms proposed was a chlamydial type III secretioneffector molecule, translocated actin-recruiting phosphoprotein (TARP), inducing chlamydial entry into the cell. TARP was discovered when a unique tyrosinephosphorylated protein was identified in infected cells and this protein increased in abundance with a concomitant increase in the multiplicity of infection (Clifton, Fields et al. 2004). This protein was absent in uninfected cells and was seen to be unphosphorylated in purified EBs. TARP localizes to the inside of the host plasma membrane at the site of chlamydial attachment and there it is phosphorylated resulting in the recruitment of actin to the site of infection. TARP phosphorylation was later seen not to be necessary in the recruitment of actin (Clifton, Dooley et al. 2005). C. muridarium, C. caviae, and C. pneumoniae TARP all lack the N-terminal phosphorylation domain yet were able to induce actin recruitment upon infection, leading Clifton et al. to propose that the $\mathrm{C}$-terminal region of the protein was responsible for actin recruitment. They suggested that TARP, though proposed to play a role in actin recruitment, may indirectly activate signal transduction pathways. Differences in the signal transduction pathways have been noted between chlamydial species (Moulder 1991) and the phosphorylation of TARP in $C$. trachomatis and not $C$. muridarium, C. caviae, and $C$. pneumoniae may be another example of varying mechanisms of signal transduction between chlamydial species. Antibodies to C. trachomatis TARP have been shown to induce protective immunity in upper genital infections of mice (Wang, Chen et al. 2009). TARP is one of several type III secretion-effector molecules produced by chlamydiae and since the discovery of a contact-dependent secretion apparatus in chlamydiae, (Hsia, Pannekoek et al. 1997), many studies have investigated the possible roles type III secretion machinery plays in respect to a chlamydial infection.

\section{Intracellular Adaptation and Survival}

Once an EB enters its target cell, several processes begin to aid its survival, differentiation, and growth. The initial objective of the EB is to avoid targeting by the lysosomal fusion pathway. Viruses and bacteria, when engulfed by a eukaryotic cell, are typically transported to the lysosome for digestion. As an intracellular pathogen, chlamydia must subvert this targeting to the lysosome to ensure its survival and subsequent growth in this environment. The chlamydial inclusion does not exhibit a drop in $\mathrm{pH}$, which is one of the initial steps in vesicles being targeted to the lysosome (Schramm, Bagnell et al. 1996). An early study (Friis 1972) noted that chlamydial escape from the lysosomal degradation pathway was dependent on live, intact 
chlamydiae. This lab also reported that heat-inactivated $C$. psittaci was phagocytized by L cells and was targeted for digestion by the lysosome (Friis 1972). However, protein synthesis by the bacteria was not necessary for prevention of lysosomal degradation, as evidenced by the ability of chlamydiae to escape targeting to the lysosome in the presence of chloramphenicol. This study concluded that entering EBs intrinsically contain factors that abrogate its digestion via the lysosomal pathway. A later study confirmed that the addition of chloramphenicol has no immediate effect on the ability of chlamydiae to avoid targeting to the lysosome to EB-containing vacuoles but that ultimately fusion occurs and EBs are degraded (Scidmore, Fischer et al. 2003). Eissenberg and Wyrick (1981) investigated whether escape from the phagolysosomal pathway was restricted to the EB-containing vacuole or was the result of a general paralysis of fusion within the infected cells. They found that co-infection of a macrophage with $C$. psittaci and Saccharomyces cerevisiae resulted in degradation of the yeast cell but not the EB, concluding that chlamydiae most likely make modifications to their inclusion membrane to avoid the lysosomal degradation pathway.

The identification of proteins localized to the inclusion membrane further proved the notion that chlamydiae modify their inclusion membrane to ensure its survival as intracellular bacteria. The Rockey lab used sera from guinea pigs infected with $C$. caviae, the agent of guinea pig inclusion conjunctivitis (GPIC), to probe by western blot lysates from both infected HeLa cells and isolated EBs to observe any differences in antigens between them (Rockey and Rosquist 1994). Three proteins, referred to as $\mathrm{p} 22$, p34, p52 (based on their molecular weights) were identified in the infected HeLa cells but absent from purified EBs and uninfected HeLa cells. It was concluded that these proteins are produced by chlamydiae and transported to the inclusion membrane during intracellular growth. A follow-up study used antisera from $C$. psittaci-infected animals to determine the cellular localization of a 39-kilodalton protein that was present in infected HeLa cells and not EBs. Using fluorescent microscopy, this protein was found in the inclusion membrane but absent from the developmental forms within the inclusion (Rockey, Heinzen et al. 1995). The protein was named inclusion membrane A (IncA). Other studies have observed and identified other inclusions-specific proteins: IncB, IncC (Bannantine, Rockey et al. 1998), IncD, IncE, and IncF, and IncG (Scidmore-Carlson, Shaw et al. 1999). Banantine et al. found that all known Inc proteins contain a similar hydrophobicity domain and took advantage of this characteristic motif to scan the $C$. trachomatis genome to identify any other potential Inc proteins (Bannantine, Griffiths et al. 2000). Forty-six potential Inc proteins were identified by this survey. Six possible candidate Inc proteins were examined to see if they localized to the inclusion membrane and five of the six proteins were confirmed to be Inc proteins. Further studies need to be performed to analyze the remaining proteins that were identified to observe if they are targeted to the inclusion membrane.

Other proteins that lack the hydrophobicity domain characteristic of the Inc proteins have also been found to be localized to the inclusion membrane. Luo et al. expressed several hypothetical proteins of the $C$. pneumoniae genome as a fusion protein with Glutathione-S-transferase (GST) and used these fusion proteins to generate antibodies in mice (2007). These antibodies were used to probe infected cells to visualize 
the localization of the corresponding protein. By confocal microscopy, the hypothetical protein, cpn0308, was seen to localize to the inclusion membrane. Western analysis identified a band only in C. pneumoniae-infected cells and not mock-infected or cells infected with $C$. trachomatis, which does not encode this protein. CT813, was also identified in this manner (Chen, Chen et al. 2006). CT813 is highly immunogenic, as evident by high titers of antibody recognizing the protein being produced from women who are urogenitally infected with $C$. trachomatis. CopN, a type III secretion-effector molecule, was also found to be localized to the inclusion membrane by indirect immunofluorescence. Heterologous expression of CopN from C. pneumoniae in both yeast and mammalian cells resulted in cell cycle arrest via disruption of the microtubule network (Huang, Lesser et al. 2008).

Some host cell proteins have been seen to be associated with the inclusion membrane. The small GTPases Rab6 and Rab11 have been observed to be associated with the chlamydial inclusion (Rejman Lipinski, Heymann et al. 2009), with Rab11 colocalizing with the chlamydial protein IncG on the inclusion surface.

Oculocerebrorenal syndrome of Lowe protein (OCRL1), a phosphatidylinositol 5phosphate that binds Rab GTPases also translocates to the chlamydial inclusion membrane (Moorhead, Jung et al. 2010). Immunofluorescence staining with antibodies revealed that annexin III, annexin IV, and annexin V all migrate to the proximity of the chlamydial inclusion (Majeed, Ernst et al. 1994). Members of the Src family of kinases are also targeted to the inclusion membrane during an infection (Mital, Miller et al. 2010). It is evident that proteins that are translocated to the chlamydial inclusion membrane are capable of interacting with host cell components, and the analysis of these proteins and their interactions may uncover specific processes that may be responsible for the pathogenesis of chlamydiae.

Within two hours of EBs entering into the host cell, the chlamydial inclusion is trafficked to the peri-nuclear region of the cell. Several studies have looked at the mechanisms that are responsible for targeting chlamydiae to this region, and it appears that the host cytoskeleton is a major factor in this process. One study observed the aggregation of F-actin and clathrin with inclusions, and this aggregation was severely disrupted with the addition of cytochalasin-D (Majeed and Kihlstrom 1991). Cytochalasin-D is a mycotoxin that inhibits actin polymerization. Using indirect immunofluorescence microscopy, Clausen et al. observed the co-localization of the host microtubule network with chlamydia-containing vesicles as chlamydiae were being trafficked to the peri-nuclear region of the cell (Clausen, Christiansen et al. 1997). It was later observed that the minus-ended microtubule motor, dynein, was responsible for this movement towards the microtubule-organizing center (MTOC) (Grieshaber, Grieshaber et al. 2003). Microinjection of antibodies directed towards a subunit of the dynein motor resulted in the abrogation of trafficking of chlamydia-laden vesicles to the MTOC, whereas antibodies that were raised towards the positive-end motor, kinesin, had no affect on this process. It was also noted that both chlamydial transcription and translation were needed in this trafficking process due to the inhibition of migration with the addition of chloramphenicol, an inhibitor of bacterial translation, and rifampicin, an inhibitor of bacterial transcription. This phenomenon suggests that the inclusion 
membrane must be modified or that an effector molecule produced by chlamydiae is imperative to achieve migration to the MTOC.

As obligate intracellular bacteria, chlamydiae require nutrients and metabolites from the host cell to ensure their growth and development. Moulder dubbed chlamydiae energy parasites that depend on the host cell for energy intermediates for the production of macromolecules (Moulder 1962). Tribby and Moulder demonstrated that chlamydiae use exogenous bases and ribonucleosides and incorporate them into their RNA and DNA during their developmental cycle (Tribby and Moulder 1966). The energy parasite hypothesis was further strengthened by Hatch when he noted that chlamydiae take up purine and pyrimidine nucleoside triphosphates from the host pools (Hatch 1975). It was also observed that thymidine uptake into chlamydia was dependent on host thymidine kinase activity (Hatch 1976). The specific mechanism by which chlamydiae obtain ATP was identified by Hatch and coworkers (1982). They found that RBs purified from infected host cells (host-free chlamydiae) use an ATP-ADP translocation system similar to that of mitochondria but in the opposite direction: ATP from the host cell cytoplasm to RBs rather than from mitochondria to the host cell cytoplasm. The Hatch lab also demonstrated that GTP is incorporated into chlamydial RNA by host-free RBs in the presence of ATP (Crenshaw, Fahr et al. 1990). Tipples and McClarty (1993) demonstrated that specific NTP requirements vary among Chlamydia spp. They found that $C$. caviae is auxotrophic for ATP, GTP, and UTP but can either obtain CTP from the host directly or convert UTP into CTP, whereas $C$. trachomatis obtains all four NTPs from the host for growth. Sequencing of the C. trachomatis genome confirmed the existence of two NTP transport systems (Stephens, Kalman et al. 1998). One of the mechanisms ultimately was shown to be the ADP-ATP translocation system first identified by Hatch et al. (1982), and the other was demonstrated to be the mechanism by which net uptake of ATP, CTP, GTP, and UTP is accomplished at the expense of ATP obtained by the translocation mechanism (Tjaden, Winkler et al. 1999). Interestingly, genome sequencing also revealed that chlamydiae are theoretically capable of generating some ATP by catabolism of glucose-6-phosphate. However, the existence of two NTP transport mechanisms suggests chlamydiae are at least partially dependent on host NTPs at some stage during the developmental cycle and, consequently, are energy parasites.

Sphingomyelin, a sphingolipid comprised of a ceramide core and phosphorylcholine side chain, is also taken up by chlamydiae (Hackstadt, Scidmore et al. 1995). In an effort to identify any interactions, cells were labeled with a Golgi-specific probe and monitored for any interactions between the chlamydial inclusion and the Golgi apparatus. It was then observed that sphingomyelin was translocated to the chlamydial inclusion and was eventually incorporated into the cell wall of the chlamydiae. Brefeldin A, a compound that disrupts retrograde transport from the Golgi to the Endoplasmic Reticulum (ER), abrogated the uptake and incorporation of sphingholipid into chlamydiae. Therefore, it was surmised that the chlamydial inclusion interrupts the natural exocytic pathway that normally targets sphingomyelin to the plasma membrane from the Golgi network. Glycoproteins, such as the vesicular-stomatitis virus G-protein, transferrin receptor, and human histocompatibility leukocyte class I antigen, were found not be translocated to the chlamydial inclusion (Scidmore, Fischer et al. 1996). Thus, 
there seems to be some specificity in which vesicles leaving the Golgi apparatus are selected and incorporated into the chlamydial inclusion. Chlamydiae also contain eukaryotic glycerophospholipids in their cell membrane, but discovering how they acquire these glycerophospholipids has been problematic. Neither treatment with Brefeldin A nor growing chlamydiae in mutant cells that lacked a lipid transfer protein abrogated the ability of chlamydiae to incorporate these eukaryotic glycerophospholipids into their membrane (Wylie, Hatch et al. 1997). More recently it has been observed that cytoplasmic lipid droplets are translocated to the lumen of the chlamydia inclusion (Cocchiaro, Kumar et al. 2008). These liquid droplets dock on the inclusion membrane, penetrate the membrane, and eventually begin to associate with RBs. This process was found to be dependent on the chlamydial proteins IncA and Lda3. This process represents a unique mechanism of organelle sequestration by an intracellular pathogen.

\section{Intracellular Growth and Development}

Once the chlamydial EBs enter a host cell and evade the host cell defenses, they reorganize to the replicative $\mathrm{RB}$ stage. The expression of chlamydial transcripts can be seen as early as one hour post-infection (Wichlan and Hatch 1993), and the expression of chlamydial proteins was observed as early as fifteen minutes post-infection (Plaunt and Hatch 1988). Most genes are expressed during the logarithmic phase and are considered to be constitutively expressed. Other genes are highly expressed at particular time points of the developmental cycle and are considered to be stage-specific. Several investigators have studied this differential pattern of gene expression in an attempt to further understand the genetic events responsible for the interconversion between the two forms of chlamydiae. Investigators used RT-PCR analysis on genes that were key components in essential biochemical pathways in an attempt to categorize the expression of genes (Shaw, Dooley et al. 2000). Seventy genes from various pathways were selected and the initiation of transcription of these genes could be categorized into three temporal classes: early stage (transcripts detectable by two hours post-infection), middle stage (transcripts not detectable at two hours but appeared by twelve hours post-infection), or late stage (transcripts detected after twelve hours post-infection). Although this study was successful in categorizing the selected genes into three different temporal classes; it only accounted for only eight percent of the genes of the $C$. trachomatis genome. Later studies would provide a more thorough examination of genetic expression in $C$. trachomatis.

Nicholson et al. performed whole genome DNA-microarray expression profiling, using transcripts produced throughout the developmental cycle, to monitor the expression of 875 C. trachomatis chromosomal genes (2003). They observed twenty-two percent of the $C$. trachomatis genome was temporally regulated. This group categorized the expression of genes into three stages. Stage I, contained genes that are expressed by twelve hours post-infection and also included the constitutively active genes. Seventytwo percent of the chlamydial genome is within this cluster of genes. The prototypic early gene, EUO, and genes responsible for DNA transcription, transport, and energy-

compound acquisition were found in this cluster. Stage II genes were defined as genes 
that transcripts increased significantly by eighteen hours post-infection. At this point during the cycle, $\mathrm{RB}$ division is at maximal levels, with few RBs differentiating back to the EB form. This cluster is comprised of genes responsible for envelope biogenesis, energy metabolism, type III secretion, protein folding, and DNA modification/recombination/repair. Stage III was defined as genes that are not expressed until twenty-four hours post-infection. At this point in the developmental cycle most RBs have reorganized to EBs or are in the process of reorganization and are preparing for exit from the host cell in order to begin another round of infection. Hypothetical proteins comprise a significant portion of genes in this group. Other genes in this cluster encode the cysteine-rich outer membrane proteins OmcA and OmcB, DNA gyrase paralogue (CT660), an orthologue of a type III secretion regulator (lcrH), and histone-like protein HctB that aids in condensing the chromosome. Major criticisms of this study are (1) critical early time points when EBs convert to RBs (within the first 5 to 10 hours of infection) were not examined; (2) RNA was isolated from purified RBs rather than infected cells, thereby possibly missing unstable RNA species; (3) RNA was not properly normalized to chlamydial DNA content.

A later study also used microarray analysis to monitor the temporal regulation of genes throughout the developmental cycle of $C$. trachomatis, but used considerably more sophisticated controls, examined very early times points, and corrected for residual late stage transcripts present in infecting EBs; this study remains the benchmark for the identification of stage-specific genes in chlamydiae (Belland, Zhong et al. 2003). Major contributions of the Belland group include identification of immediately early stage genes and the importance of differential tryptophan synthesis genes and EUO gene expression during interferon gamma-induced persistence during the developmental cycle. The mechanisms that regulate temporal expression of chlamydial genes are an ongoing focus in the field.

\section{Exit}

The final step in the chlamydial developmental cycle is release from the host cell. Using live imaging of McCoy cells infected with $C$. trachomatis serovar E to monitor the nature of the chlamydial inclusion during interphase and mitosis, researchers set out and observed the lysis of the host cells at seventy-two hour post-infection (Campbell, Richmond et al. 1989). Similarly, C. trachomatis infection of the primary cell cultures of the human amnion resulted in the rupture of host cells at late times in the infection (Neeper, Patton et al. 1990). Investigators (Rockey, Fischer et al. 1996) evaluated $C$. psittaci inclusions during an infection and noticed the lysis of the inclusion, followed by the infectious EBs occupying the cell cytoplasm, then the final step of cell lysis and the escape of the EBs to begin another round of infection. This study also noted that one of the lobes of the chlamydial inclusion escaped from the cell before lysis, indicating that chlamydia may possess multiple mechanisms of escape from the host cell.

Other studies have noted chlamydiae escaping the host cell by a means other than lysis. Fluorescent-antibody staining of HeLa cells infected with C. trachomatis serovar D 
showed that the release of chlamydiae from the cell was not concomitant with the death of the cell. Ultrastructural studies showed the chlamydial inclusion was transported to the host cell surface via a process similar to exocytosis (Todd and Caldwell 1985). Another group (Hybiske and Stephens 2007) examined the method of release by taking advantage of the ability of chlamydial inclusions to exclude large, soluble fluorescent markers. They stably expressed green fluorescent protein (GFP) in HeLa cells thereby making the chlamydial inclusions visible via live fluorescence microscopy and reached the conclusion that EB release occurs via one of two mechanisms: by lysis of the host cell or by a packaged release called extrusion. Cellular regulators of both the cell lysis and the extrusion pathways were identified in this study. Cell lysis was dependent on calcium signaling within the cell, and treatment of the cell with protease inhibitors abrogated the lysis process. Extrusion required actin polymerization, myosin II, and Rho GTPase. Similar to the attachment process, it is likely that different species of Chlamydia use different mechanisms to exit host cells and that method of exit may vary with environmental conditions even within a given species.

In summary, the chlamydial developmental cycle begins with the attachment of the EB to the target cell and entry into cytoplasm by only partially understood mechanisms that resemble endocytosis and which include avoidance of the nascent inclusion into the lysosomal pathway. Within a few hours, possibly a few minutes, the EB initiates early gene expression necessary for conversion to RBs, which divide by binary fission. After growth and division RBs convert back to EBs which are released by multiple mechanisms to infect new cells. All of these processes require chlamydiae to express genes in an organized manner. The environmental signals which trigger stagespecific expression and the mechanisms by which stage-specific regulation is accomplished are topics at the forefront of chlamydial research.

\section{GENE REGULATION}

The mechanisms by which temporal gene expression is regulated in chlamydiae is poorly understood. However, several mechanisms of chlamydial gene regulation in general have been elucidated.

\section{Promoters and Sigma Factors}

Because chlamydiae progress through morphologically distinct developmental stages (in the very simplest terms: EB-to-RB, early stage; RB cell division, middle stage; RB-to-EB, late stage), it follows that chlamydial gene expression is temporally regulated. The first specific gene products demonstrated to be stage-specific were two cysteine-rich proteins (CRPs), now known to be encoded by an operon designated omc AB. The CRPs were identified by SDS-PAGE analysis in EBs but not in dividing RBs (Hatch, Allan et al. 1984). In 1986, two additional EB-specific proteins, now known to be positively charged histone-like proteins HctA and HctB, were reported to bind to negatively

charged host cell membranes and speculated to be chlamydial adhesins (Hackstadt 1986). 
In the absence of a tractable genetic system and prior to sequencing of the late-stage encoding genes, regulatory mechanisms responsible for stage-specific expression could only by speculated. In other bacteria, stage-specific and environmentally induced expression of genes is often accounted for by the expression of different sigma factors. Sigma factors bind to the RNA polymerase and enable it to preferentially bind specific promoter sequences. Bacteria may possess multiple sigma factors, as found in B. subtilis, $S$. coelicolor, and cyanobacteria, that function in a cascade-like fashion to differentially regulate genes during their developmental cycles (Gruber and Gross 2003). A particular sigma factor may be responsible for the regulation of other sigma factors, thereby initiating a "cascade" of sigma factor expression. Even before any chlamydial sigma factors were identified, a "sigma cascade" was a favored hypothesis for temporal expression in chlamydiae.

The first chlamydial sigma factor to be identified was designated sigma 66 on the basis of its molecular mass. Upon sequencing, the sigma 66 gene (rpoD) was observed to encode a protein highly homologous in amino acid sequence to the major sigma factors of E. coli (sigma 70) and B. subtilis (sigma 43) (Koehler, Burgess et al. 1990). Sigma 66 was found to be present in RBs and EBs and consequently deemed to initiate the expression of "house-keeping" genes, as is the case for sigma 70-like factors in other bacteria.

The first chlamydial gene to be cloned and sequenced was ompA, encoding the major outer membrane protein (MOMP) (Stephens, Kuo et al. 1985). Putative promoters were identified upstream of the two MOMP gene transcripts, neither of which were found to be expressed in E. coli. This led to the premature conclusion that chlamydial promoters were "different" from other bacterial promoters (Stephens, Wagar et al. 1988). However, 5'end analysis of two chlamydia cryptic plasmid transcripts, both with the same 5'end, revealed near-consensus like -10 and -35 promoter elements (TTGCCA and TATATT, respectively) (Fahr, Sriprakash et al. 1992). Investigators (Douglas, Saxena et al. 1994) developed a chlamydial in vitro transcription system using the plasmid template cloned into a transcription assay vector, and later in vitro studies confirmed that recombinant sigma 66 recognizes the most distal ompA promoter (Fahr, Douglas et al. 1995). The translation start site proximal transcript, with only GC-rich upstream sequence, was determined to be a post-transcriptional product of the long transcript. In vitro mutagenesis coupled with in vitro transcription analysis found that whereas the ompA promoter (-10: CACAAA) is not recognized by $E$. coli sigma 70, promoter sequences more closely resembling the bacterial consensus (-10: TATAAT) are also efficiently recognized by recombinant sigma 66. An exhaustive in vitro mutagenesis-in vitro transcription study of the rRNA operon by Tan and Engel (1996) confirmed that the preferred Sig66 promoter -35 and -10 sequences are identical to the E. coli sigma 70 consensus (TTGACA and TATAAT), although sigma 66 is clearly more promiscuous than other bacterial major sigma factors (Mathews and Sriprakash 1994). Several other genes, not considered to be cycle-specific, have been experimentally shown by in vitro transcription analysis to be controlled by either sigma 66 or recombinant $E$. coli sigma 70: the $k d s A$ gene (Brabetz and Brade 1997), the tuf gene (Shen, Shi et al. 2000), the 
dnaK gene (Schaumburg and Tan 2003), and the genes of the type III secretion system (Hefty and Stephens 2007).

A total of three sigma factors, sigma 66 , sigma 28 , and sigma 54 , were identified upon sequencing the chlamydia genome, lending strength to the hypothesis that a sigma cascade, most likely sequential expression of sigma 54/sigma 28, might be responsible for temporal gene regulation in chlamydiae (Stephens, Kalman et al. 1998). Sigma 54 and sigma 28 are found in many bacteria, and the genes they regulate differ depending upon the bacterial species. These alternative sigmas have yet to be implicated directly in the transcription of developmental genes in other bacteria, although activation of sigma 54 in response to environmental stimuli has been shown to trigger stalk and fruiting body formation in Caulobacter and myxococcus, respectively (McCleary and Zusman 1990; Marques, Gomes et al. 1997).

Researchers used host-free generated RNA probes to identify late-stage specific genes, including the previously identified $h c t A$ and $o m c A$ and two new late genes designate ltuA and ltuB (Fahr, Douglas et al. 1995). They identified the 5' ends of these genes and also the remaining known late stage gene $h c t B$ and located likely promoters on the basis of homology to known bacterial promoter sequences; all of the predicted promoters most closely resembled the bacterial major sigma consensus. They used a chlamydial in vitro transcription system, coupled with inhibitory anti-sigma 66, to demonstrate that all but $h c t B$ were under the control of sigma 66, erecting a significant barrier to sigma cascade hypothesis for late-stage gene expression. A later group found that the Fahr et al. study had misidentified the transcription start site of $h c t B$ and presented evidence that transcription of this gene is initiated by sigma 28 , re-kindling the idea of alternative sigma factors playing a role in at least late-stage gene regulation $(\mathrm{Yu}$ and Tan 2003).

Sigma 28 is responsible for the transcription of genes required for flagella biosynthesis (Helmann 1991) and chemotaxis (Mirel, Lustre et al. 1992) in other bacteria, but these genes are lacking in Chlamydia. To identify potential sigma 28-regulated genes in chlamydiae, the genome was scanned for promoter sequences that resembled the canonical sigma 28 consensus sequence, since this sequence is highly conserved among bacteria (Yu and Tan 2003). After scanning the genome, a sequence closely resembling the sigma 28 consensus was identified upstream of $h c t B$. Recombinant sigma 28 was produced and reconstituted in a chlamydial in vitro transcription system with which the expression of the $h c t B$ promoter region was assessed and found to be responsible for transcription from the predicted sigma 28 promoter. Mutational analysis was also performed on the $h c t B$ promoter region in an effort to define the sequences that were optimal for sigma 28 binding and for the purpose of better predicting sigma 28 binding regions within the chlamydial genome (Yu, Di Russo et al. 2006). One group later used a probability weight matrix based on known sigma 28 promoters of other bacteria with the new information they gathered from the mutational analysis of the $h c t B$ promoter to predict other possible sigma 28 regulated genes (Yu, Kibler et al. 2006). Sixteen additional candidate genes were identified, but functional analysis via in vitro transcription showed only five of these candidate genes were actually under the control of 
sigma 28: tsp, dnaK, tly C_1, bioY, and pgk. Primer extension analysis was used to validate the location of the putative sigma 28 promoters in vivo. The genes $h c t B$, $t s p$, and pgk mapped to within six nucleotides of 5' ends, and dnaK mapped to within 10 nucleotides of predicted start site. Of these newly identified sigma 28 controlled genes, three of these genes are known to be expressed late within the developmental cycle: $h c t B$, tsp, $t l y C_{-} 1$ (Belland, Nelson et al. 2003). This would suggest that sigma 28 may regulate genes expressed late in the developmental cycle. In contrast bioY, pgk, and dnak were seen in the Belland et al. study to be express early within the developmental cycle. It was noted that the Belland et al. study monitored only the steady state transcription levels and would not be able to distinguish the temporal activity of genes with multiple promoters; $p g k$ was regulated by sigma 28 and sigma 66 in this study and dnaK was regulated by sigma 28 in this study and sigma 66 in a previous study (Wilson and Tan 2002). So it is possible that sigma 28 regulation of genes is restricted to late time points; no other study has indicated that sigma 28 regulates genes expressed early during the developmental cycle.

In some bacteria, such as Salmonella and B. subtilis but not E. coli, sigma 28-like sigma factor activity is controlled by regulators. These sigma factor regulatory proteins include RsbV, RsbW, and RsbU; all of which are encoded by Chlamydia spp., with the curious addition of a second RsbV protein. The sigma regulators use a partner-switching mechanism to allow sigma 28 to bind or not bind RNA polymerase in response to environmental stimuli, usually some form of stress (Brown and Hughes 1995). When RsbW binds to sigma 28, it prevents sigma 28 binding to RNA polymerase (Kang, Brody et al. 1996). RsbV proteins are kinases that bind to RsbW and phosphorylate the protein; this phosphorylation inhibits the binding of RsbW to sigma 28. RsbU is a phosphatase that dephosphorylates RsbW, thereby allowing RsbW to once again bind the sigma 28 . In the absence of a usable genetic system, the chlamydial anti-sigma factors were examined in a heterologous system (Salmonella enterica serovar typhimurium) (Karlinsey and Hughes 2006). Karlinsey and Hughes used C. trachomatis sigma 28 to complement a $S$. enteric sigma 28 mutant and assayed its activity by measuring motility. C. trachomatis sigma 28 was seen to complement the mutant strain and restored motility. This group introduced the $C$. trachomatis sigma 28 negative regulator, $\mathrm{RsbW}$, in the system and observed that the sigma 28 activity was unaffected. Hua et al. also examined the role of these conserved partner switching proteins by utilizing in vitro kinase reactions and yeast two-hybrid assays to assess the ability of these proteins to bind one another (2006). They observed both RsbU and RsbW binding RsbV, but similar to the Karlinsey and Hughes study, they did not observe RsbW binding of sigma 28. It was concluded that the chlamydial Rsb proteins may function in a manner similar to those in Bordetella. Bordetella bronchiseptica contains the partner switching proteins BtrU, $\mathrm{BtrV}$, and BtrW and the interactions between these proteins were examined (Kozak, Mattoo et al. 2005). Generating mutations in serine 55 of BtrV (target of BtrW phosphorylation) and asparagine 51 of BtrW (pivotal for kinase activity) allowed this group to characterize the interactions between the two proteins. Surprisingly, neither mutation affected the ability of these proteins to bind. Using these mutations of BtrV and BtrW, they then monitored their effects on the type III secretion apparatus of Bordetella. It has been reported in Bordetella that the deletion of btrV or btrW (Mattoo, Yuk et al. 
2004) results in the disruption of polypeptides being excreted through the type-III secretion apparatus. Each mutation of BtrV and BtrW resulted in the inability of the type-III secretion apparatus to secrete proteins. They proposed that phosphorylation of $\mathrm{BtrV}$ by BtrW is essential for the activation of the type-III secretion machinery and once $\mathrm{BtrW}$ disrupts its binding with BtrV by phosporylating the protein, it is then free to associate with an alternative regulatory protein that would allow the secretion of proteins through the type III secretion apparatus. This study provides an alternative mechanism of action for the partner switching paradigm. The partner-switching mechanism of sigma 28 regulation in Chlamydia should be further examined in an effort to better define its role in chlamydial gene regulation.

Sigma 54 is the second alternative sigma factor encoded by Chlamydia spp. genomes. In many bacteria, including E. coli, sigma 54 coordinates the expression of genes related to nitrogen metabolism, most significantly, up-regulation of the glutamine synthetase gene $g \ln A$ during nitrogen starvation. In that chlamydiae lack $g \ln A$, one group (Mathews and Timms 2000) conducted in silico studies to find potential sigma 54 promoters within the genome, taking advantage of the fact that the cognate sigma 54 promoter sequences are the most highly conserved of all bacterial sigma factor binding sequences (Barrios, Valderrama et al. 1999). Using the FindPatterns program, they were able to identify nine promoters that match the sigma 54 consensus sequence (allowing two mismatches) that were located within four hundred base pairs upstream of a chlamydial open reading frame. Primer extension analysis was performed to establish the chlamydial start sites of these genes. Only two of the nine predicted promoter regions produced a transcript that mapped close to the actual 5'ends: open reading frames CT652.1 and CT683. CT652.1 and CT683 are hypothetical genes with no known function, but orthologs with sigma 54-like promoters were also identified in the genomes of C. muridarum, and C. pneumoniae. Also noted was the presence of an upstream activator sequence (TGT-N10/11-ACA) to which the NtrC DNA-binding protein is critical for activation of expression of sigma 54 genes (Kustu, Santero et al. 1989). NtrC is the DNA binding member of a two-component gene regulatory system that also includes the sensor protein, NtrB. Genes encoding homologs ( $c t c C$ and $c t c B)$ are encoded by chlamydial genomes, but curiously the NtrC-protein equivalent lacks an obvious DNA binding motif, although the protein has been demonstrated to be phosphorylated by the NtrB equivalent, CtcB (Koo and Stephens 2003). Sigma 54 controlled genes also require binding of integration host factor (IHF) to an upstream DNA element to promote a bend in DNA that allows interaction of NtrC with sigma 54 (Hoover, Santero et al. 1990). Two putative IHF elements were identified upstream of both the CT652.1 and the CT683 promoter regions, and Zhong et al. (2001) demonstrated that recombinant chlamydial IHF binds to DNA upstream of the omcAB operon (binding upstream of CT652.1 and CT683 has not been tested). To date, an in vitro transcription system using recombinant chlamydial sigma 54 has not been achieved because the recombinant is insoluble (T.P. Hatch, personal communication 1997); hence, experimental proof that sigma 54 regulates CT652.1, CT683, or any other gene is lacking. 
In conclusion, the roles for the alternative sigma factors in controlling stagespecific transcription are unsettled. At least some late-genes possess sigma 28 promoters, but most do not. Thus, a simple sigma cascade cannot explain temporal gene regulation in chlamydiae. The role of Rsb proteins in temporal regulation, including sigma 28 activation, remains unclear, and the function of sigma 54 and putative auxillary proteins remains a complete mystery. A better characterization of when sigma and auxillary genes and proteins are expressed during the cycle would be useful in support or elimination of their roles in temporal expression.

\section{DNA Supercoiling}

Supercoiling of DNA can be a method of regulating the expression of genetic material in bacteria. Depending on the superhelicity of the DNA, gene expression can either be upregulated or downregulated. A decrease in the superhelicity of DNA may result in either the expression or the repression of a particular gene or subset of genes. The cause of this upregulation or downregulation of gene expression is believed to be due to the change of promoter activity and efficiency (Dorman 2006). The change in the DNA structure via supercoiling may cause the inability of DNA-binding factors such as transcription activators or suppressors to not be able to effectively bind the DNA, thus causing the disruption or the enhancement of transcription. It was discovered that the DNA topology downstream of the ilvGMEDA operon of $E$. coli (which expresses five of the six enzymes needed to produce L-isoleucine and L-Valine) regulates the expression of the ilvGMEDA operon (Pagel, Winkelman et al. 1992). Subsequent studies identified an element, which they termed the supercoiling-induced DNA duplex destabilized structure (SIDD) that when bound by the integration host factor (IHF) protein produced a change in the superhelicity of the -10 element of the downstream ilvGMEDA operon, resulting in the transcription activation of this operon by increasing the rate of open complex formation (Sheridan, Benham et al. 1998).

Because of studies that have presented DNA topology as a factor in gene expression, chlamydial researchers have examined chlamydia for similar genetic regulation via DNA topology. Differences in superhelicity of chlamydial DNA were first noticed when the C. trachomatis cryptic plasmid was isolated from EBs and RBs and fractionated on a gel. Different topoisomers of the plasmid were observed in EBs compared to RBs (Solbrig, Wong et al. 1990). A highly supercoiled topoisomer was unique to the $\mathrm{EB}$, whereas a less supercoiled topoisomer was unique to the RB. Differences between the two plasmid isolates were denoted by both gel migration patterns and by appearance using electron microscopy. The differences in the superhelicity of the plasmid lead researchers to hypothesize that the DNA topology may play a role in the inert nature of EBs vs. active gene expression in RBs.

Others addressed the role of DNA topology in regulating gene expression in chlamydiae by examining the in vitro activity of five chlamydial promoters (Niehus, Cheng et al. 2008). They examined two genes ompA (major outer membrane protein) and $p g k$ (phosphoglycerate kinase), which are expressed during the mid-cycle stage of 
development, and three genes, omcAB (cysteine-rich proteins), hctB (histone-like protein), and $l t u B$ (late transcription unit B), which are expressed during the late stage of the cycle for their responsiveness to modifications to the superhelicity of their promoter regions. It was observed that expression of mid-cycle genes omp $A$ and $p g k$ was increased by as much as fifty-fold and eight-fold, respectively, with increased negative supercoiling of their promoters. In contrast, the late genes $\operatorname{omc} A B, h c t B$, and $l t u B$ all exhibited a modest increase in transcription levels when their promoter regions were subjected to an increase in negative supercoiling. The extent of supercoiling of the DNA was examined during the developmental cycle by Niehus et al. by isolating the chlamydial plasmid at different times post-infection and subjecting the plasmid to gel fractionation. They found that the plasmid was more negatively supercoiled at the mid-cycle stage compared to both the early and the late stages of the developmental cycle. The increase in transcription seen in negatively supercoiled DNA, combined with the evidence that chlamydial DNA appears to be more negatively supercoiled during the mid-cycle stage of infection when gene expression is high, further substantiates the notion that changes in DNA topology is a mechanism by which Chlamydia species may regulate gene expression. In a follow-up study, in vitro transcription analysis to monitor the change in expression of stage-specific genes of the type III secrection machinery when the degree of supercoiling of the template plasmid was altered (Case, Peterson et al. 2010). They observed that the type III secretion genes that transcribed during middle stages of the developmental cycle in vivo increased its expression when negative supercoils are introduced to DNA, whereas genes that are expressed late in the developmental cycle in vivo expressions were insensitive to the induction of negative supercoils, further validating the hypothesis that DNA topology has an effect on genetic expression. It is unclear how closely the in vitro transcription system relates to in vivo conditions and what in vivo processes might be responsible for changes in supercoiling of the chlamydial genome.

\section{Small Regulatory RNA}

Another mechanism by which bacteria regulate gene expression is by small regulatory RNAs (sRNA). sRNAs are defined as untranslated RNAs that are less than four hundred nucleotides long and regulate both transcription and translation (Wassarman and Steitz 1992) by base-pairing with other RNA molecules or by binding proteins. Studies in E. coli have revealed the presence of seventeen non-coding RNAs that possess a functional role in gene regulation (Gottesman, Storz et al. 2001). For example, oxyS RNA, induced during oxidative stress, represses the translation of the $f h l A$ gene by blocking ribosome binding (Altuvia, Zhang et al. 1998). In contrast, $d s r A$ RNA positively regulates the expression of the $r p o S$ gene (Sledjeski, Gupta et al. 1996).

Initial evidence that chlamydiae may contain non-translated, regulatory RNAs came from studies investigating the transcription of the $7.5 \mathrm{~kb}$ cryptic plasmid located in the $C$. trachomatis serovar L2 (Fahr, Sriprakash et al. 1992). Two transcripts of 250 and 430 bases with a common 5 ' end were identified with predicted bacterial major sigma factor promoter -10 and -35 elements. Both transcripts lacked a strong ribosome-binding 
site, which suggested that these transcripts may not be translated during the developmental cycle. It was also noted that transcripts were located anti-sense to the 3' end of a predicted transcript that encodes for a recombinase-like protein. Expression of the recombinase-like gene could not be detected by Northern blot or primer extension analysis but was found by RT-PCR amplification. It was suggested but not demonstrated that the two short anti-sense transcripts abrogated the expression of this gene therefore presenting the first indication of the regulation of gene expression via short non-coding RNAs in chlamydiae.

The chlamydial histone-like proteins, HctA and HctB, also known as Hc1 and Hct2, are made late in the developmental cycle and bind to chlamydial DNA to induce the electron dense nucleoid seen by microscopy in EBs (Costerton, Poffenroth et al. 1976). In an attempt to identify the mechanism by which hctA expression is regulated, a heterologous system was constructed in E.coli where they screened plasmids containing chlamydial gene library in an effort to find genes that would rescue the lethal phenotype when hctA is expressed in E. coli (Grieshaber, Grieshaber et al. 2006). They discovered two loci that would rescue $E$. coli from lethality. One was the $i s p E$ gene that synthesizes the metabolite, non-mevalonate methylerythritol 4-phospate (MEP). The authors speculated that this charged metabolite binds to the positively charged histone protein to release HctA from genomic DNA. A fault with this reasoning is that ispE appears to be expressed late in the cycle rather than at immediately early times. The second locus contained a small RNA species, designated ihtA, which inhibited the translation of hctA RNA; thereby reducing HctA levels thus rescuing the lethal phenotype. In $C$. trachomatis, the levels of ihtA increases by four hours post-infection and reach peak levels at twelve hours post-infection; this correlated with the decreased expression of HctA. The protein levels of HctA begin to increase around twenty-four hours with a concomitant drop in $i h t A$ expression thus validating the initial evidence that it is a negative regulator of hetA expression.

A global search for sRNA species in C. trachomatis identified forty non-coding RNAs (Abdelrahman, Rose et al. 2011). Chlamydia has been observed to produce noncoding RNAs and this group used an Affymetrix microarray containing the $C$. trachomatis serovar D genome in an effort to identify novel non-coding RNAs. Forty non-coding RNAs were identified and a subset of these non-coding RNAs was subjected to further analysis; these non-coding RNAs were analyzed based on their expression levels at twenty-four hours, forty-eight hours, and their expression during IFN- $\gamma$ persistence. These non-coding RNAs were seen to have a distinct temporal expression pattern and this expression pattern was altered when a persistent infection was induced. This study provided evidence for the role of non-coding RNAs regulating genetic expression in Chlamydia and sets the framework for future studies regarding non-coding RNAs and the roles they play in genetic regulation. 


\section{DNA-Binding Proteins}

It has long been known that the binding of proteins to DNA can result in the upregulation or downregulation of genes. These proteins work in a myriad of ways. They can direct RNA polymerase binding to specific regions of the genome and help aid the initiation of transcription. These proteins can bind to UP elements upstream from the promoter, causing a bend in the DNA to increase the rate of transcription. They can also bind the DNA in such a manner that abrogates transcription. Predicted DNA binding proteins are not common in chlamydial genomes, but several have been identified and analyzed for their effect on gene expression.

Ultrastructural studies noted that DNA within the RB form of chlamydiae was in a fibrous, loosely organized "reticulate" state, whereas the EB form contained DNA in a compacted, condensed nucleoid state (Costerton, Poffenroth et al. 1976). Investigators identified three DNA-binding proteins of 58, 25, and $17 \mathrm{kDa}$ that were specific to EBs and suggested they might be responsible for the compaction of DNA EBs (Wagar and Stephens 1988). Genes encoding two predicted highly basic proteins were eventually identified (Hackstadt, Baehr et al. 1991; Brickman, Barry et al. 1993). DNA sequence analysis of one of the encoding genes revealed an amino acid composition similar to the eukaryotic $\mathrm{H} 1$ histone, as well as sharing primary sequence homology to the $\mathrm{H} 1$ family of histones (Hackstadt, Baehr et al. 1991). This grouped named the protein Hcl (HctA), which is expressed during the later stages of the developmental cycle. An antigenically related histone analog, termed Hc2 (HctB), was also identified and analyzed (Brickman, Barry et al. 1993). Expression of $h c t A$ gene in E. coli resulted in the compaction of its chromosome similar to the condensation of the chlamydial chromosomes. Both of the chlamydial histone-like proteins have been observed to restrict gene transcription in E.coli. The late expression of these proteins, along with its ability to bind and condense chromatin material, is believed to be responsible for the transcriptional inactivity of EBs.

In an attempt to identify proteins that were capable of binding the upstream region of the cysteine-rich protein operon, $o m c A B$, one lab fractionated RB extracts on a gel, eluted proteins from the gel slices, and incubated the eluates with the promoter region of the operon (Zhong, Douglas et al. 2001). Gel mobility analysis revealed a protein between 13 and $16 \mathrm{kDa}$ that caused a shift when allowed to bind the $o m c A B$ operon. Matrix-assisted laser desorption/ionization time of flight (MALDI-TOF) mass spectrometric analysis revealed the presence of a protein with high similarity to the alpha and beta subunits of the integration host factor of other bacteria. Further analysis of this protein revealed that it was able to introduce a bend in the CRP operon promoter region and a two-fold increase in transcription was observed when measured via in vitro transcription. The IHF protein was produced during the middle and late stages of the developmental cycle, which corresponds with an increase in the expression of the CRPs. How and if the chlamyidal IHF alters expression of the CRP operon in vivo has not been determined. A unique feature of chlamydiae is that their genomes encode only one of the hetero-dimeric IHF proteins seen in other bacteria. 
The chlamydial genome also contains a homologue of the heat-shock gene

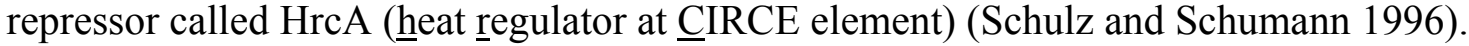
HrcA binds to the cis-acting DNA element, CIRCE (controlled inverted repeat of chaperone expression), which is located upstream of the promoters to the heat shock dnaK and groE operons (Tan, Wong et al. 1996). It was later shown that the association of $\mathrm{HrcA}$ to the CIRCE element caused a repression of $d n a K$ promoter activity via in vitro transcription analysis (Wilson and Tan 2002). In another study, investigators observed an element upstream of the groES/groEL promoter, but with a sequence that is highly divergent from known bacterial CIRCE elements. In vitro analysis reveal weaker binding of HcrA protein to the groES/EL CIRCE element than to the dnaK element and also weaker repression of transcription (Wilson and Tan 2004). It was also noted that HcrA protein repressed in vitro expression of the late-stage, non-stress gene $h c t A$ when and only when the $d n a K$ CIRCE element was placed upstream of the gene.

Iron is a necessary element for all living organisms. It is a key cofactor of several enzymes that are responsible for the oxidation of many compounds. Organisms must take up this essential element to ensure survival. Growing chlamydiae in host cells under iron-starved conditions results in a decrease in infectivity, a delay in development, abnormal morphological forms, and quantitative changes in the expression levels of several proteins (Raulston 1997). The changes seen in protein expression during irondeprivation in bacteria are regulated by a family of repressors that are homologous to the E. coli ferric uptake regulator (FUR) protein. A protein with limited sequence homology to the FUR-protein was identified in C. trachomatis and named divalent cation-dependent regulator A (DcrA) (Wyllie and Raulston 2001). DcrA was demonstrated to complement an E. coli strain that was mutated so that its FUR protein was not expressed. Follow-up studies were performed to try and identify other sequences in the genome that may be under the control of the DcrA (Rau, Wyllie et al. 2005). In this study, the E. coli FUR protein was used to scan the chlamydial genome to identify sequences that were capable of being bound by the protein using a FUR titration assay (FURTA). Twenty-eight sequences were identified and were tested via electric mobility shift analysis (EMSA) to see if they were capable of being bound by the chlamydial DcrA protein. DcrA protein was able to bind to these sequences although further experimentation, such as in vitro transcription, need to be conducted in an effort to validate DcrA function.

It is believed that the genes that are expressed early during the developmental cycle are responsible for the conversion of the EB to the RB. Before the chlamydial genomes were sequenced, Wichlan and Hatch attempted to identify genes that are preferentially expressed during the early stages by making use of a unique feature of chlamydiae: the ability of host-free RBs to incorporate highly radioactive, exogenous GTP into the same mRNA that had been initiated and were in the process of elongation when the RBs were present in infected host cells (1993). The radioactive host-free RNA was used to probe a genomic library, and a clone was selected on the basis of its reaction with a two-hour probe but not a probe generated at 24-hours. The identified gene encoded a hypothetical protein unique to chlamydiae; it was named early upstream open reading frame or EUO because of its early expression and its location upstream of $g t l X$, a gene predicted to encode glutamyl-tRNA synthetase. EUO gene sequences revealed a 
positive net charge in the N-terminus and the hydrophobic C-terminal tail, suggesting that it could possibly be a DNA-binding protein. Further studies revealed that EUO transcripts are detectable as early as one hour post-infection, are more highly expressed relative to $r p o D$ and rRNA at one to two hours post-infection, but continue to be expressed during the logarithmic phase of growth, whereas expression sharply declines at later stages of the developmental cycle (Zhang, Douglas et al. 1998). The protein was not found in infectious EBs, suggesting that it is not stable. A putative weak helix-turn-helix DNA-binding motif was predicted from the EUO gene sequence and to assess the DNAbinding activity of EUO, reverse gel-mobility shift assays were carried out. Radiolabeled recombinant EUO was allowed to incubate with EcoRI-digested chlamydial DNA and resolved on a non-denaturing polyacrylamide-agarose gel. Recombinant EUO failed to enter the gel in the absence of DNA fragments but was able to enter the gel in the presence of multiple fragments chlamydial genomic and BlueScript plasmid DNA, indicating that it is indeed capable of binding DNA in a non-specific manner. The EUO gene is located in close proximity of the $o m c A B$ operon and gel-shift analysis verified that EUO was capable binding to the promoter region of this operon, and DNA footprint studies showed that EUO preferred sequences of DNA that were highly AT-rich. Binding of EUO to oligonucleotide probes confirmed the preference of EUO to bind to AT-rich but not GC-rich sequences. The intriguing possibility that EUO may function to repress transcription of late genes was raised; however, in vitro transcription studies found that EUO repressed expression of the mid-cycle $о m p A$ gene, as well as the $o m c A B$ operon---likely because of the relatively non-specific binding of EUO to AT-rich sequences in vitro.

A later study performed a genome-wide search in an effort to define sequences of DNA in the chlamydial genome to which EUO prefers to bind (Zhang, Howe et al. 2000). A technique called SELEX (systematic evolution of ligands by exponentially enrichment) was employed. This method used enzyme-digested chlamydial DNA to construct a library of possible EUO binding targets. The DNA was ligated into pBluescript and allowed to incubate with recombinant EUO. The mixture of recombinant protein and vector was allowed to pass through membranous filter, with unbound DNA passing through the filter and EUO-bound DNA being retained. This DNA/protein hybrid was removed from the membrane and the DNA portion of this complex was isolated and subjected to amplification in E. coli. This process was repeated through nine rounds. Several clones were selected using this method with the most highly enriched clone containing sequence within the coding region of the deoxycytidine triphosphate deaminase $(d c d)$ gene. DNase footprint analysis of this selected clone showed that EUO was preferentially binding AT-rich sequences. With the alignment of the selected clones, the weak DNA-binding consensus sequence AHGAAAWVTYTWDAY was derived, but it required at least two mismatches to identify all the strong binding sequences in the clones selected. Although this study located genomic sequences to which EUO preferred to bind in vitro, the sequences themselves provided no insight into the role of EUO in vivo. More global in vitro and in vivo studies are needed to further define the role of EUO in chlamydiae. One curious finding suggested that EUO is a histone H1-specific protease (Kaul, Hoang et al. 1997). 


\section{OBJECTIVE}

The developmental cycle of Chlamydia spp. is governed by a gene regulatory system that is not fully understood. The conversions of the EB to the RB and the RB back to the EB have been described ultrastructurally but the molecular events that are responsible for these intercoversions have not been fully characterized. Although the lack of a stable genetic transformation system has hampered progress, several mechanisms of genetic control have been identified. The purpose of this study was to look at two possible mechanisms of genetic control: sigma factor regulation and EUO DNA-binding regulation, and to further characterize their roles in the regulation of chlamydial gene expression. Sigma factor protein expression during the developmental cycle will be examined in Chapter 2, in vitro binding of recombinant EUO to genomic DNA will be analyzed by ChIP-to-Chip analysis in Chapter 3, and in vivo binding of native EUO to DNA will be examined by an immunoprecipitation-cloning strategy in Chapter 4. 


\section{CHAPTER 2. PROTEIN EXPRESSION LEVELS OF SIGMA 66, SIGMA 54, AND SIGMA 28 THROUGHOUT THE DEVELOPMENTAL CYCLE OF CHLAMYDIA}

\section{INTRODUCTION}

Sequencing of the chlamydial genome revealed the presence of three sigma factors: sigma 66, the major sigma factor, and two alternative factors, sigma 54, and sigma 28 . The discovery of putative alternative sigma factors stimulated the speculation that they may play a decisive role in temporal regulation of chlamydial gene expression. In 2000, the Hatch lab (Douglas and Hatch 2000) used reverse transcription polymerase chain reaction (RT-PCR) to monitor the transcript levels of these sigma factors to assess if they are expressed in a cascade-like fashion. Sigma 66 transcripts were present throughout the developmental cycle although transcripts could not be detected in elementary bodies. Sigma 54 and sigma 28 transcripts exhibited similar expression profiles whereby their transcript levels were low during the early stages of the developmental cycle and expressions peaked during the logarithmic phase of growth. These data suggested that the alternative sigma factors of Chlamydia are unlikely to function strictly at the promoters of either late or early stage genes. Various in silico studies have predicted which genes may be regulated by which sigma factors, and in vitro transcription studies have confirmed these predictions for a handful of genes. The first in vitro study to examine transcription of all late stage genes identified at the time found that their transcription is initiated from sigma 66 promoters (Fahr, Douglas et al. 1995). However, in vitro studies from the Tan lab have identified several genes, including one of the late stage histone-like genes, are initiated from sigma 28 promoters ( $\mathrm{Yu}$ and Tan 2003), reintroducing the possibility of alternative sigma factors playing a critical role in stage-specific gene expression.

Previous studies have focused on monitoring the transcript levels of the sigma factors. In a study investigating transcription and translation during chlamydial persistence, investigators observed an increase in transcript expression of one stagespecific gene, EUO, without a concomitant increase in protein expression (Ouellette, Hatch et al. 2006). The mechanism responsible for this uncoupling between transcription and translation is not known but suggests the presence of sigma transcripts at a given stage of the cycle may not correlate with functional proteins. Therefore, the objective of the studies described in this chapter was to monitor sigma factor protein production during the cycle. The working hypothesis is that analysis of sigma protein levels during the cycle may identify one of the alternative sigma factors as a potential regulator of stage-specific gene expression. Chlamydia-infected cells were probed with antibodies raised against the sigma factor proteins and their expression levels were monitored via immunofluorescence assays. Western blot analysis of extracts of purified reticulate bodies (RBs) was used to confirm the immunofluorescence results. One of the sigma factors, sigma 28 , was identified as a potential regulator of gene expression in the latelogarithmic phase of growth. 


\section{MATERIALS AND METHODS}

\section{Growth of Chlamydia Trachomatis Serovar L2}

L929 mouse fibroblasts were grown with M199 in suspension culture. $4.0 \times 10^{8}$ cells were infected with C. trachomatis serovar L2. $3.5 \times 10^{5}$ of the infected cells were collected and allowed to grow in Leighton tubes containing microscope slides.

\section{Antibodies}

Rabbit polyclonal antibodies were provided by Annemarie Douglas in the Hatch lab. They were commercially generated from gel-purified recombinant $C$. trachomatis L2 sigma 66, sigma 28, sigma 54 and EUO proteins by Dr. Douglas. Monoclonal mouse anti-sigma 54 was provided by the Guangming Zhong lab, the University of Texas San Antonio HSC. Monoclonal anti-MOMP and polyclonal anti-CRP were provided by Harlan Caldwell, Rocky Mountain Laboratories.

\section{Isolation of Chlamydiae}

Infected L cells in spinner culture were collected and chlamydial RBs and elementary bodies (EBs) were harvested at 10, 16, 24, 36, and 48 hours post-infection (hpi). Spinner cultures were spun down at 1,000 rpm for five minutes to collect the infected cells. The cell pellet was suspended in $10 \mathrm{mLs}$ of PBS (Phosphate Buffered Saline). The infected cells were lysed via bead sonication in a sonic water bath (Meddleton Electronics). The lysed material was allowed to incubate with DNase (2 $\mathrm{mg} / \mathrm{mL}$ ) for thirty minutes at a temperature of $37^{\circ} \mathrm{C}$. This material was spun down at $10,000 \mathrm{rpm}$ for 20 minutes in a Beckman Coulter centrifuge. The resulting pellet was suspended in $10 \mathrm{mLs}$ of PBS and spun down at 1,000 rpm for 5 minutes. The supernatant was then collected and loaded on a Renocal three-step gradient consisting of $30 \%, 35 \%$, and $40 \%$ layers. This gradient was spun down in an ultracentrifuge at $25,000 \mathrm{rpm}$ for 35 minutes at a temperature of $4^{\circ} \mathrm{C}$. RBs were isolated from the $30 \% / 35 \%$ interface and EBs were isolated from the $35 \% / 40 \%$ interface. This material was spun down in microcentrifuge tubes in an Eppendorf centrifuge at 10,000 rpm for 10 minutes. The supernatant was discarded and the resulting pellet was resuspended in $100 \mu \mathrm{Ls}$ of gel digest mix with a final concentration of $5 \%$ beta-mercaptoethanol.

\section{Sodium Dodecyl Sulfate-Polyacrylamide Gel Electrophoresis and Western Blot Analysis}

Preparations of extracts from the various time points $(10,16,24,36$, and $48 \mathrm{hpi})$ were boiled for 5 minutes. The samples were then loaded and resolved in one dimension on a $13 \%$ polyacrylamide gel with sodium-dodecyl sulfate buffer. Sample volumes were 
loaded and adjusted such that the amount of major outer membrane protein (MOMP) at each time point was the same. The samples were electrophoresed for one hour at a constant voltage of 200 volts. The gel was then subjected to western blot analysis. The proteins were transferred to a polyvinylidene fluoride (PVDF) membrane. The membrane blocked with a $10 \%$ milk solution and was probed overnight with rabbit polyclonal antibodies directed towards sigma 66 , sigma 54 , sigma 28 , EUO, or the cysteine-rich protein complex protein, $\mathrm{OmcB}$. The dilution of all primary antibodies used was 1:1,000. After overnight incubation with primary antibodies, the membranes were washed three times with PBST with fifteen minute agitations. After the washes, the membrane was incubated with a 1:20,000 dilution of horse radish peroxidase-conjugated donkey $\operatorname{IgG}$ in for 4 hours. After this incubation, the membrane was washed once with PBST and three times with PBS with fifteen minute agitations. Signal was visualized via chemiluminescence with the Immun-Star HRP chemiluminescent kit from Bio-Rad. Kodak Biofilm was used in development.

\section{Immunofluorescence Analysis (IFA)}

Chlamydial proteins were visualized within infected $\mathrm{L}$ cells via immunofluorescence microscopy. Leighton tubes containing $3.5 \times 10^{5} \mathrm{~L} 2$-infected cells grown on coverslips were fixed with methanol for 48 hours. After fixation, the cells were blocked for 10 minutes with PBS containing bovine serum albumin. The slides were then incubated with antibodies raised against sigma 66 , sigma 54, sigma 28, EUO, or CRP for 1 hour at $37^{\circ} \mathrm{C}$. Slides were washed with PBS and were allowed to incubate with donkey anti-rabbit antibody conjugated to an Alexa fluor for 1 hour at $37^{\circ} \mathrm{C}$. This process was repeated for the MOMP counter-stain. The counter stain antibody was detected with a goat anti-mouse antibody conjugated to a FITC fluor. The coverslips were examined using an oil-immersion phase lens on an Axiovision confocal microscope.

\section{RESULTS}

\section{Strategy of Analysis}

Protein levels were monitored by IFA of infected cells and western blot analysis of extracts of purified chlamydiae. Each method has its strengths and weaknesses. IFA allows examination of individual inclusions, including at early time points. However, the method is semi-quantitative at best and highly subject to observer interpretation. In my studies, I attempted to photograph images at the same intensity as I observed through the optical lens. Another problem is the potential problem of access of the primary antibody into late RBs and EBs because of disulfide cross linkage of the outer membrane complex $(\mathrm{OMC})$ late in the cycle. However, this latter problem is mitigated by the speculated incomplete cross-linkage in intracellular chlamydiae (Hatch, Allan et al. 1984). Western blot analysis was normalized by loading lanes with a constant amount of the constitutive major outer membrane protein (Figure 2-1), and thus is more quantitative than IFA. 


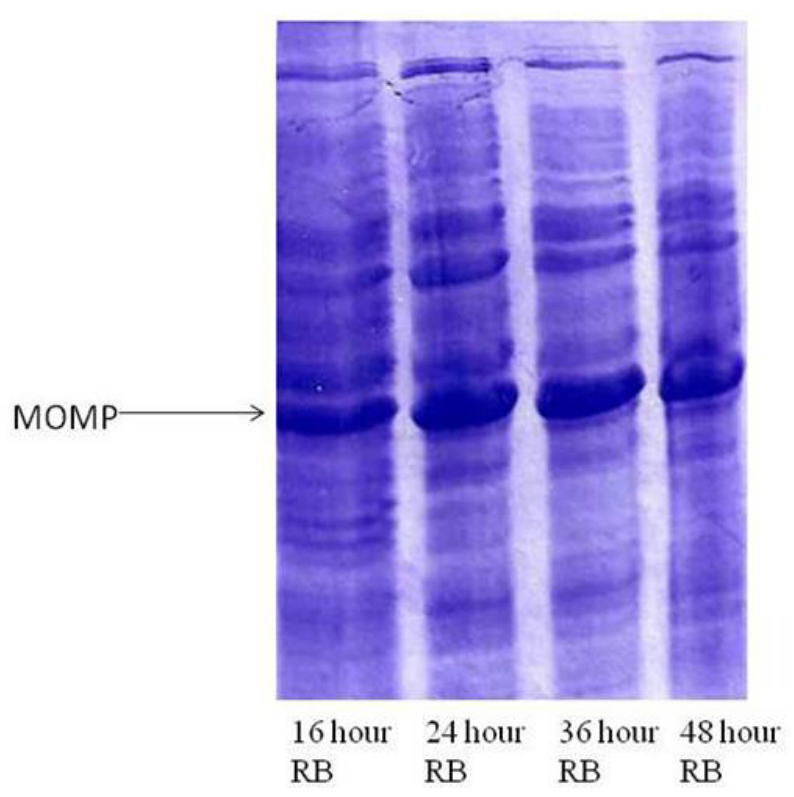

\section{Figure 2-1. Coomassie stain of reticulate bodies.}

Reticulate bodies (RB) harvested from 16, 24, 36, and 48 hours post-infection were lysed and loaded on a $13 \%$ polyacrylamide gel. The amount of extract loaded was adjusted so that the amount of MOMP at each time was the same, providing a loading control for the IFA studies reported in the remaining western blot Chapter 2 figures. 
However, it is extraordinarily difficult to obtain sufficient amounts of chlamydial extract not contaminated with interfering host material at early time points. In my studies, I found $16 \mathrm{hpi}$ to be the earliest time point yielding consistent results.

\section{Sigma 66 Is Expressed throughout Developmental Cycle}

The protein expression level of sigma 66 was monitored using IFA and western blot analysis. The time points chosen were 10, 16, 24, 36, and $48 \mathrm{hpi}$, thereby encompassing the early (10 hpi), middle (16 and $24 \mathrm{hpi})$, and late stages (36-48 hpi) of the developmental cycle. Due to difficulties in obtaining sufficient material at early time points, the 10 hour time point was omitted in western blot analysis. Sigma 66 protein was detected as early as 10 hpi by IFA (Figure 2-2). At this time point, chlamydia inclusions were very small and completed either no rounds or only a few rounds of division. To determine if the sigma 66 protein was chlamydia-specific and not due to background noise, monoclonal antibodies to MOMP were used as a counter-stain. In these experiments, the sigma 66 staining overlapped with the MOMP counter-stain and was absent throughout the rest of the field, indicating that the sigma 66 protein was confined to the chlamydial inclusion. Sigma 66 protein was also observed also at the 16 and 24 hour time points, suggesting that the protein is present throughout the logarithmic phase of the chlamydial growth. Sigma 66 staining at the 36 and 48 hour time points appeared less intense and was speckled throughout the inclusion, suggesting that the expression of sigma 66 may be declining during the time of RB-to-EB conversion.

To confirm the IFA results, western blot analysis was performed (Figure 2-3). Similar amounts of sigma 66 protein were detected in the 16, 36, and 48 hour samples, suggesting the level of sigma 66 is present in roughly equal amounts throughout the logarithmic and late stages of growth. I attribute the lack of detection of sigma 66 protein in the 36 and 48 hour immunofluorescence samples to the partial cross-linking of the $\mathrm{OMC}$ in late RBs and in EBs, thereby impeding antibodies from entering the chlamydia. The surface-exposed MOMP is not subject to a similar restriction. The slight increase in sigma 66 expression at 24 hour sample in the western analysis may be attributed to inaccurate loading of material because all proteins examined at this time point exhibited a peak in expression (Figure 2-1).

In summary, sigma 66 protein was observed early, during the logarithmic phase of growth, and at late times post-infection --- supporting the hypothesis that sigma 66 may function in the initiation of stage-specific as well as house-keeping genes in Chlamydia.

\section{Sigma 54 Is Expressed throughout the Developmental Cycle of Chlamydia}

Sigma 54 protein expression was monitored by IFA throughout the course using rabbit polyclonal antibody directed against gel-purified recombinant $C$. trachomatis L2 sigma 54. The protein was observed as early as $10 \mathrm{hpi}$ (Figure 2-4). At this time point, staining was dull and diffuse but present in every inclusion. Sigma 54 protein increased 


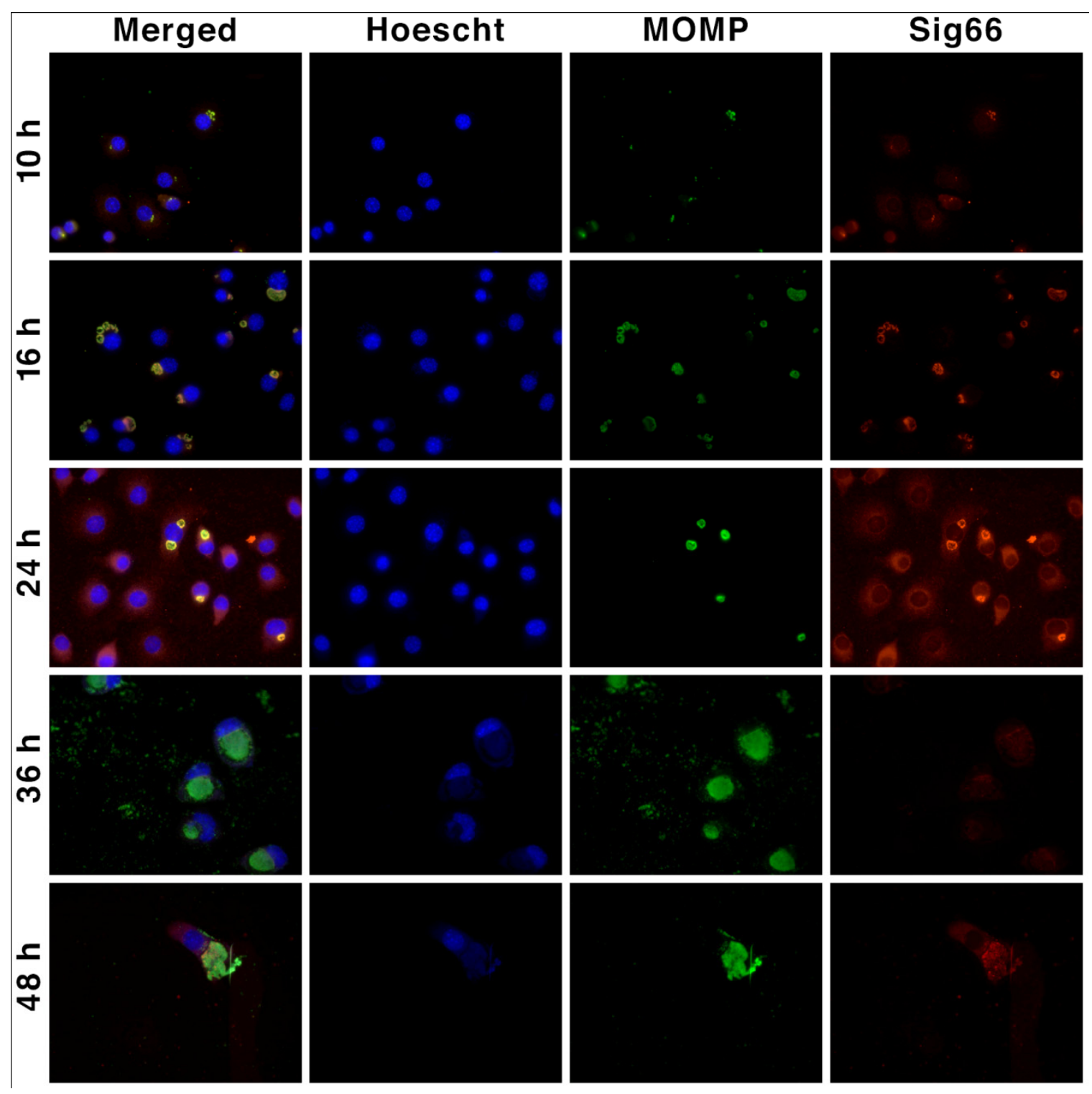

Figure 2-2. IFA of sigma 66 in C. trachomatis-infected cells.

Infected cells were fixed with $\mathrm{MeOH}$ for 48 hours at the times indicated. The cells were reacted with rabbit polyclonal sigma 66 and monoclonal MOMP antibodies. Hoescht was used to stain DNA. 


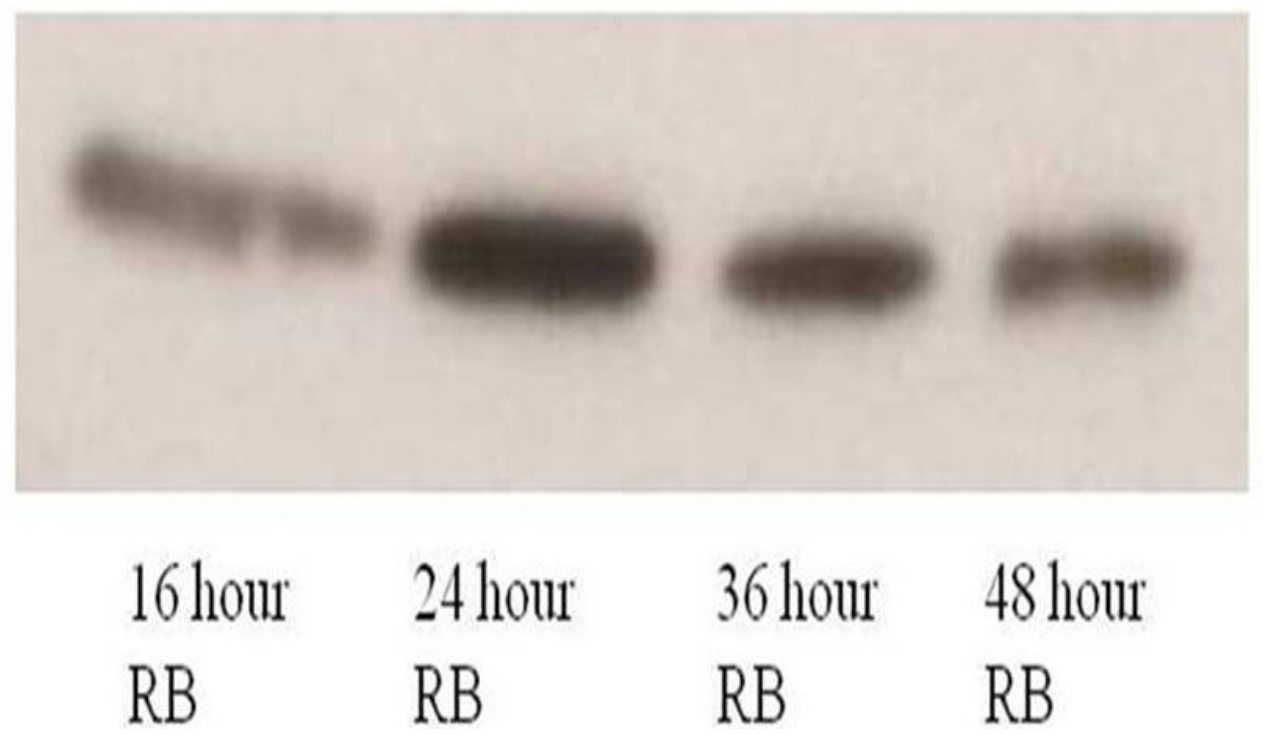

Figure 2-3. Detection of sigma 66 protein in reticulate bodies.

Western blot analysis was performed on RBs isolated at 16, 24, 36, and 48 hpi. Rabbit polyclonal serum generated against sigma 66 was used to probe the extracts. MOMP was used as a load control. Protein standards (not shown) indicated the reactive bands shown were of a relative molecular mass of $66 \mathrm{kDa}$. 


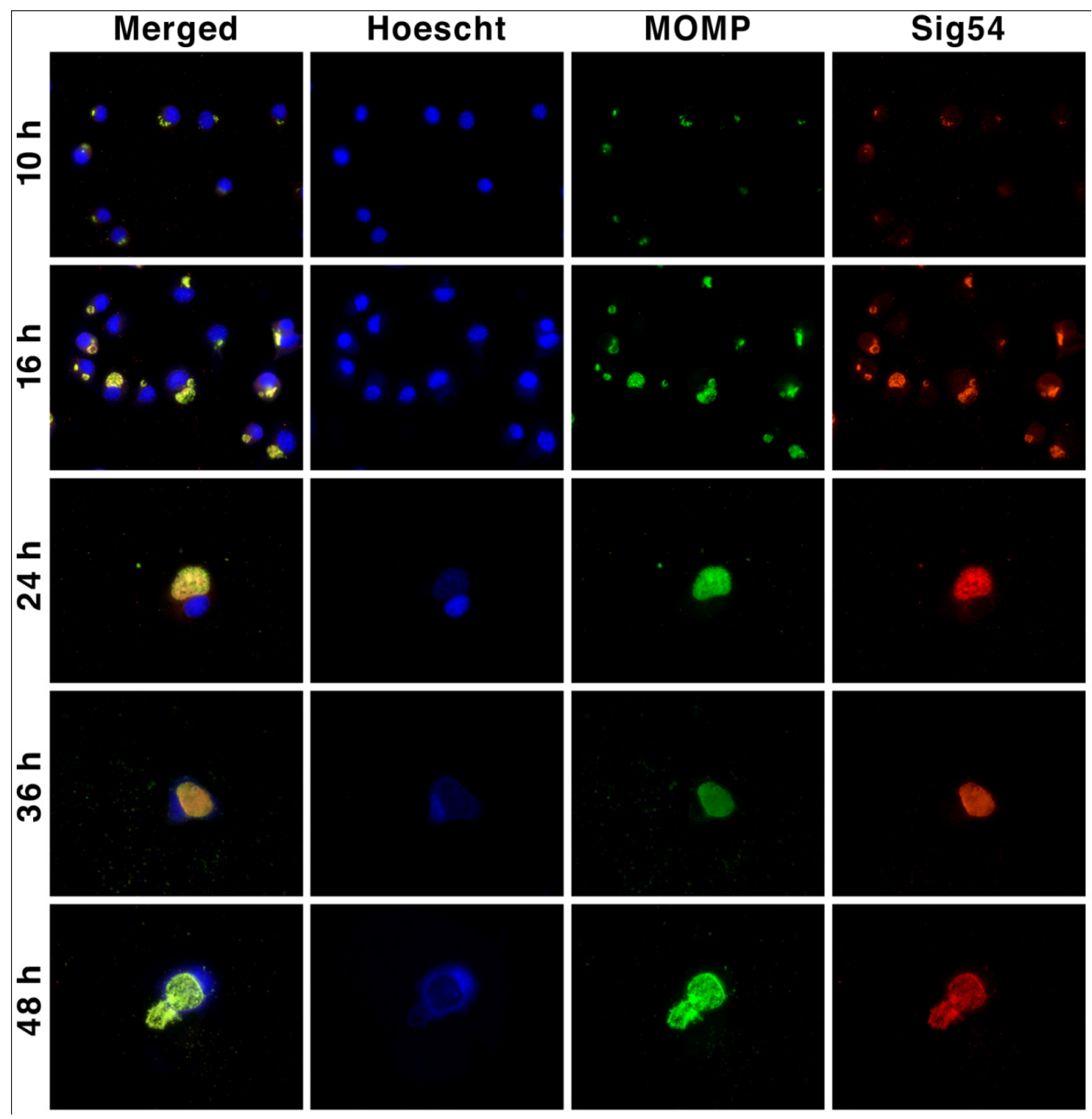

Figure 2-4. IFA of sigma 54 in $C$. trachomatis-infected cells.

Infected cells were were fixed with $\mathrm{MeOH}$ for 48 hours at the times indicated. The cells were reacted with rabbit polyclonal anti-sigma 54 and monoclonal MOMP antibodies. Hoescht was used to stain DNA. 
from the 10 hour to the 16 hour time point. Curiously, close examination of the inclusions at 16 and 24 hpi revealed that the pattern of sigma 54 staining appeared similar to the MOMP and associated with the OMC rather than the cytoplasm of RBs, the expected location of a sigma factor. This odd observation will be further investigated later in this chapter. The level of sigma 54 did not noticeably change throughout the cycle from 16 to $48 \mathrm{hpi}$, but remained very high. To verify these results, sigma 54 was monitored by western blot analysis (Figure 2-5). For reasons that are unclear, detection of sigma 54 antigen by western analysis of chlamydial extracts using the polyclonal antiserum was inconsistent, often yielding blurred bands and a high background. Of the dozen or more gels run, the one shown in Figure 2-5 was the best. In the blot shown in Figure 2-5, bands were detected at all times, with a sizable increase between 16 and 24 hpi. About the same amount of material was detected from 24 to 48 hpi. In summary, the western blot and IFA studies were consistent, with sigma 54 detected at relatively early through late times hpi, suggesting that this sigma factor could function at any time from 10 to $48 \mathrm{hpi}$.

\section{Sigma 28 Is Expressed during the Logarithmic and Early Late Phases of Growth}

Sigma 28 protein levels were monitored from 10 to 48 hpi by IFA (Figure 2-6). At 10 hpi, the staining was dull, not clearly present in every inclusion and may represent background staining. At 16 hpi, sigma 28 protein staining was relatively bright and completely overlapped with the MOMP counter-stain, indicating its presence in every inclusion. At $24 \mathrm{hpi}$, the intensity of the sigma 28 stain was similar to the $16 \mathrm{hpi}$ stain but it was restricted to the periphery of the inclusion. Similar to the sigma 66 antibody, the possibility of sigma 28 antibody being blocked from entering the elementary bodies due to the cross-linkage of its outer membrane cannot be overlooked. At 36 and 48 hours post-infection, the expression of sigma 28 began to decline as evident by the decrease in staining intensity and the absence of sigma 28 stain from most inclusions.

Sigma 28 protein was detected by western analysis in the earliest time point examined, 16 hpi (Figure 2-7). Protein levels increased from the 16 to the 24 hours and thereafter declined but did not disappear. In summary, the IFA and western blot studies suggest sigma 28 protein level is absent or at very low levels early during the logarithmic phases of growth, peaks at about 24 hpi and declines thereafter. In all the experiments described to this point, only RBs were detected at 24 hours, both by IFA and in the purification gradients. The 24 hpi time most likely represents the late log phase of the cycle, just before EBs are formed. Taken altogether, sigma 28 most likely is not present in significant amounts at early log phase and late in the cycle. Thus, if sigma 28 functions in some way to regulate temporal gene expression, it most likely does so at the beginning of RB to EB transition.

\section{Polyclonal Sigma 54 Cross-Reacts with an Antigen in the OMC}

As described above, the Hatch lab rabbit polyclonal antibody that was generated against chlamydial sigma 54 reacts inconsistently on western blots of chlamydial extracts 


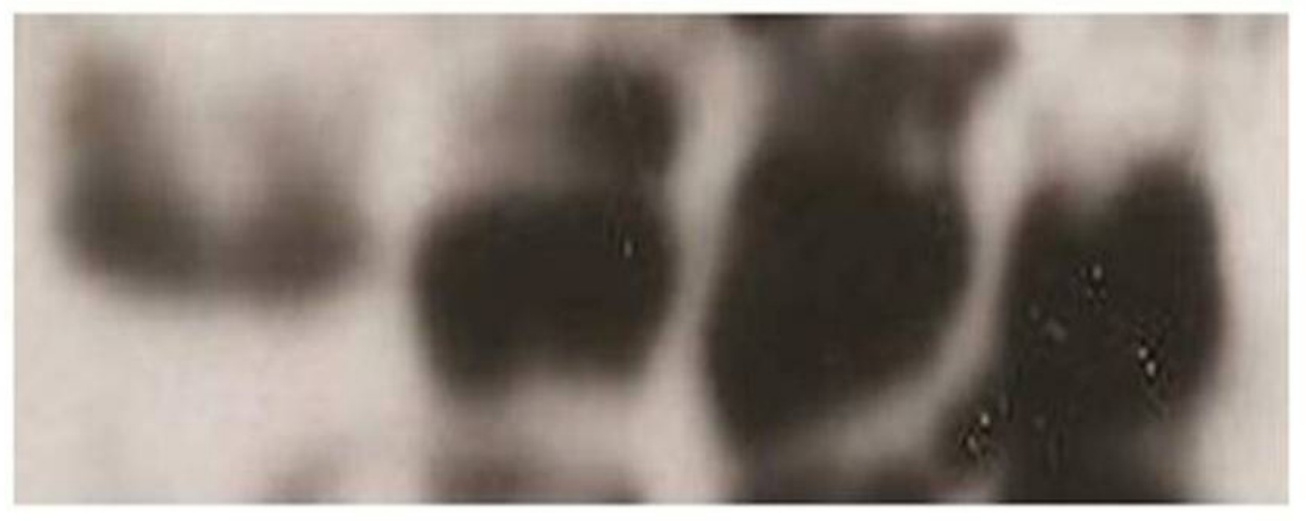

\section{6 hour \\ 24 hour 36 hour \\ 48 hour \\ $\mathrm{RB}$ \\ $\mathrm{RB}$ \\ $\mathrm{RB}$ \\ $\mathrm{RB}$}

Figure 2-5. Detection of sigma 54 in RBs by western blot analysis.

Western blot analysis was performed on RBs isolated at 16, 24, 36, and 48 hpi. Rabbit polyclonal serum generated against sigma 54 was used to probe the blot. MOMP was used as a load control. Protein standards (not shown) indicated the reactive bands shown were of a relative molecular mass of $45 \mathrm{kDa}$. 


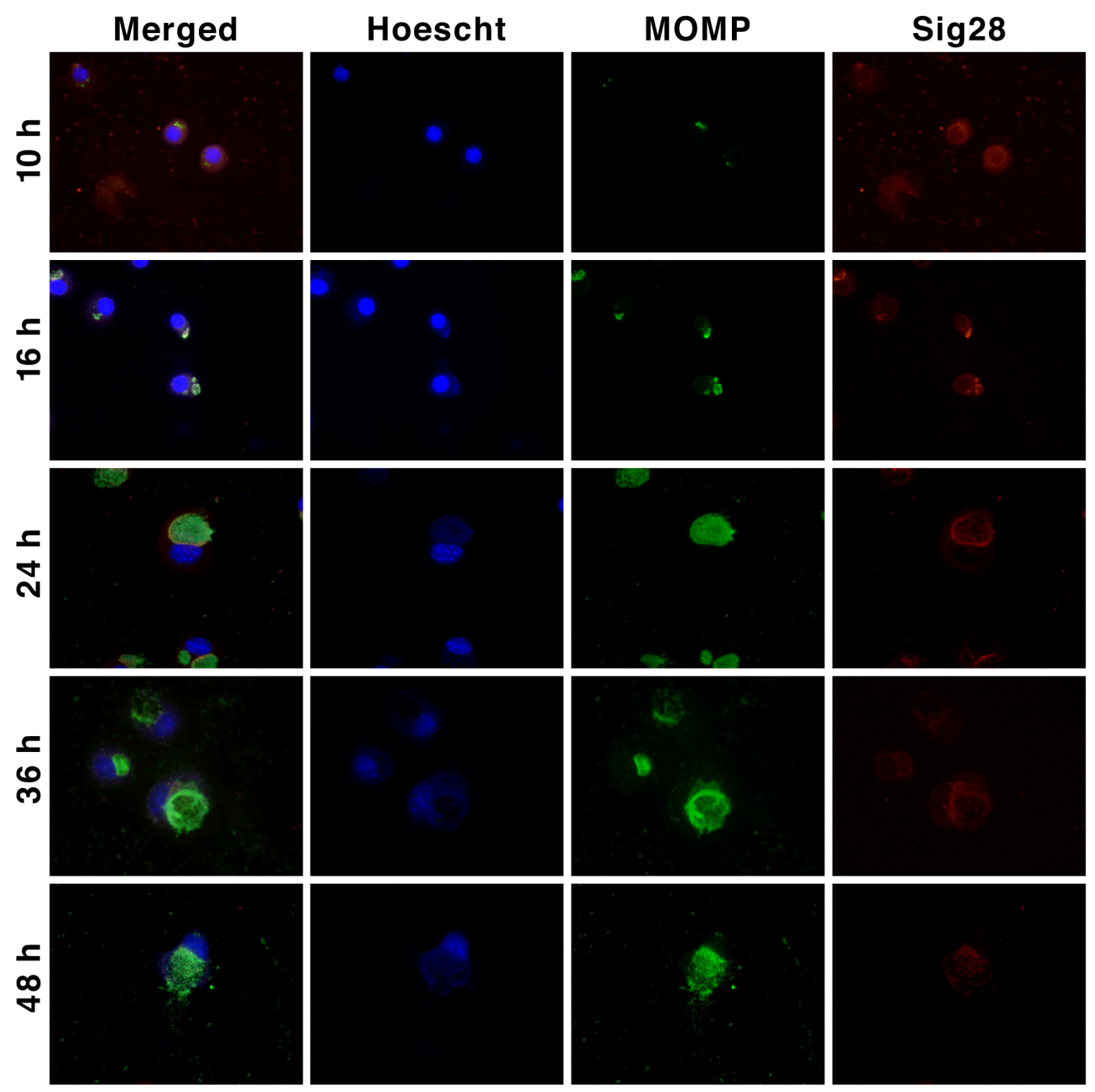

Figure 2-6. IFA of sigma 28 in $C$. trachomatis-infected cells.

Infected cells were fixed with $\mathrm{MeOH}$ for 48 hours at the times indicated. The cells were reacted with rabbit polyclonal anti-sigma 28 and monoclonal MOMP antibodies. Hoescht was used to stain DNA. 


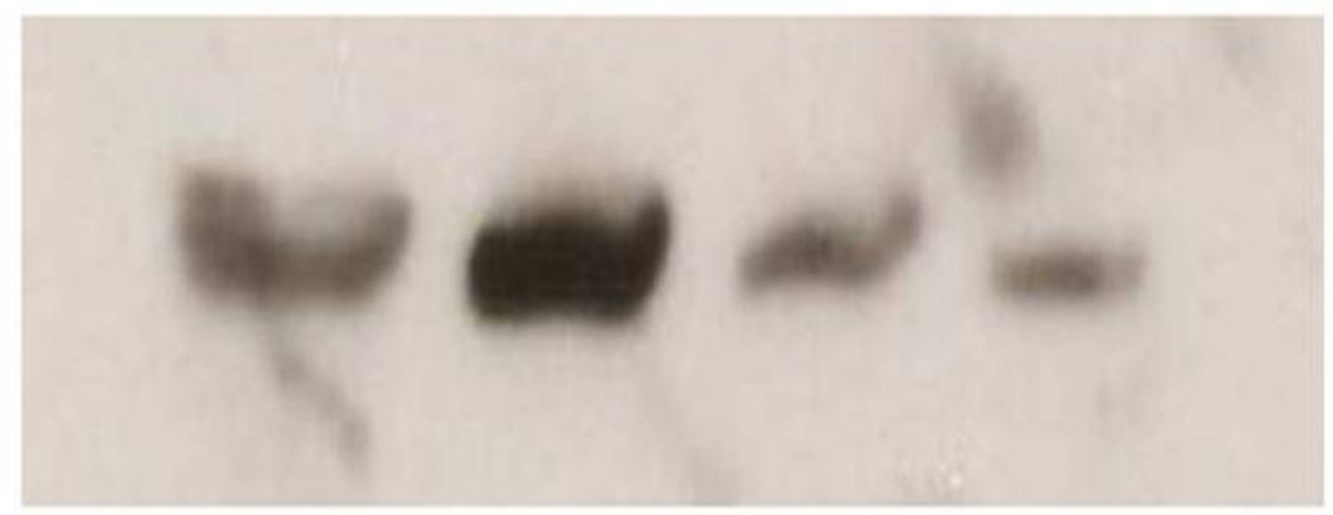

\section{6hour 24hour 36hour 48hour $\mathrm{RB} \quad \mathrm{RB} \quad \mathrm{RB} \quad \mathrm{RB}$}

Figure 2-7. Detection of sigma 28 in RB extracts by western blot.

Western blot analysis was performed on RBs isolated at 16, 24, 36, and 48 hpi. Rabbit polyclonal serum generated against sigma 28 was used to probe the extracts. MOMP was used as a load control. The reactive band migrated with a relative molecular mass of 28 kDal. 
and reacts in IFA with a pattern consistent with reaction with the OMC. Recombinant sigma 54 is highly insoluble, which may be a factor in the peculiar reaction of the antibodies. However, the antibody does react with recombinant sigma 54 by western blot and antibody purified from western blots of recombinant protein continues to react with the perimeter of RBs (Thomas Hatch, personal communication 2003). To further explore the problem of the cellular location of sigma 54, the Hatch lab recombinant protein was sent to the Guangming Zhong lab for generation of a mouse monoclonal antibody. However, the recombinant protein failed to generate antibody that reacted with infected cells. Consequently, the Zhong lab generated a soluble recombinant sigma 54 from a GST-tagged truncated sigma 54 protein. This protein was used to boost one time the mouse previously inoculated with the Hatch lab recombinant. The following studies were undertaken to compare the reactions of the polyclonal and monoclonal antibodies. L cells were infected with C. trachomatis serovar L2 and samples were obtained at 10, $16,24,36$, and $48 \mathrm{hpi}$. IFA was performed with the sigma 54 rabbit polyclonal and mouse monoclonal antibodies (Figure 2-8). IFA was also performed using the mouse monoclonal sigma 54 antibodies, polyclonal EUO, an early stage marker, and CRP, a late-stage marker (Figure 2-9). At the $10 \mathrm{hpi}$, the inclusions were small, but there was complete overlap of the reaction of the two sigma 54 antibodies (Figure 2-8). Overlap was also observed in the 16-hour time point and the intensity of the staining increased from the 10-hour sample. Differences in staining between the two antibodies first appeared in the 24-hour time point, when the polyclonal serum continued to stain the entire inclusion and the monoclonal antibody began to be more concentrated around the periphery of the inclusion. There were areas within the interior of the inclusion that stained brightly with the polyclonal serum but either stained weakly with the monoclonal antibody or lacked the monoclonal stain altogether. This trend continued in the 36 and 48 hour samples. Also, the intensity of the monoclonal antibody stain decreased at later times. RBs are located at the periphery of the inclusion, and it is widely believed that dividing RBs are attached to the inclusion membrane and that as RBs convert to EBs, they become detached and relocate to the interior of the inclusion. The data shown in Figure 2-9, in which the monoclonal sigma 54 reactions are compared to EUO and CRP, confirms that the antigen recognized by the monoclonal is present as early as $10 \mathrm{hpi}$, when EUO is also present, and very late in the infection, when CRP is present. A notable point, however, is that at very late times, the sigma 54 antigen is concentrated in RBs still attached to the inclusion membrane, whereas the CRP marker is intense throughout the inclusion.

It is possible that sigma 54 protein is present in two forms: one form found in the interior of RBs, recognized well by the monoclonal antibody and poorly by the polyclonal, and a second form in the OMC of RBs and EBs, recognized well by the polyclonal but not the monoclonal. To explore this possibility, IFA was performed on purified EBs, which were allowed to adhere to a microscope slide and fixed with methanol for 48 hours. A control experiment was performed using MOMP antibodies to confirm that EBs did adhere to the slide and that the small "EB dots" were not specs of background (data not shown). The procedure was repeated using the polyclonal serum anti-sigma 54 as the primary stain and the monoclonal antibody as the counter-stain. The polyclonal serum stained the EBs brightly and the monoclonal antibody stain was mostly 


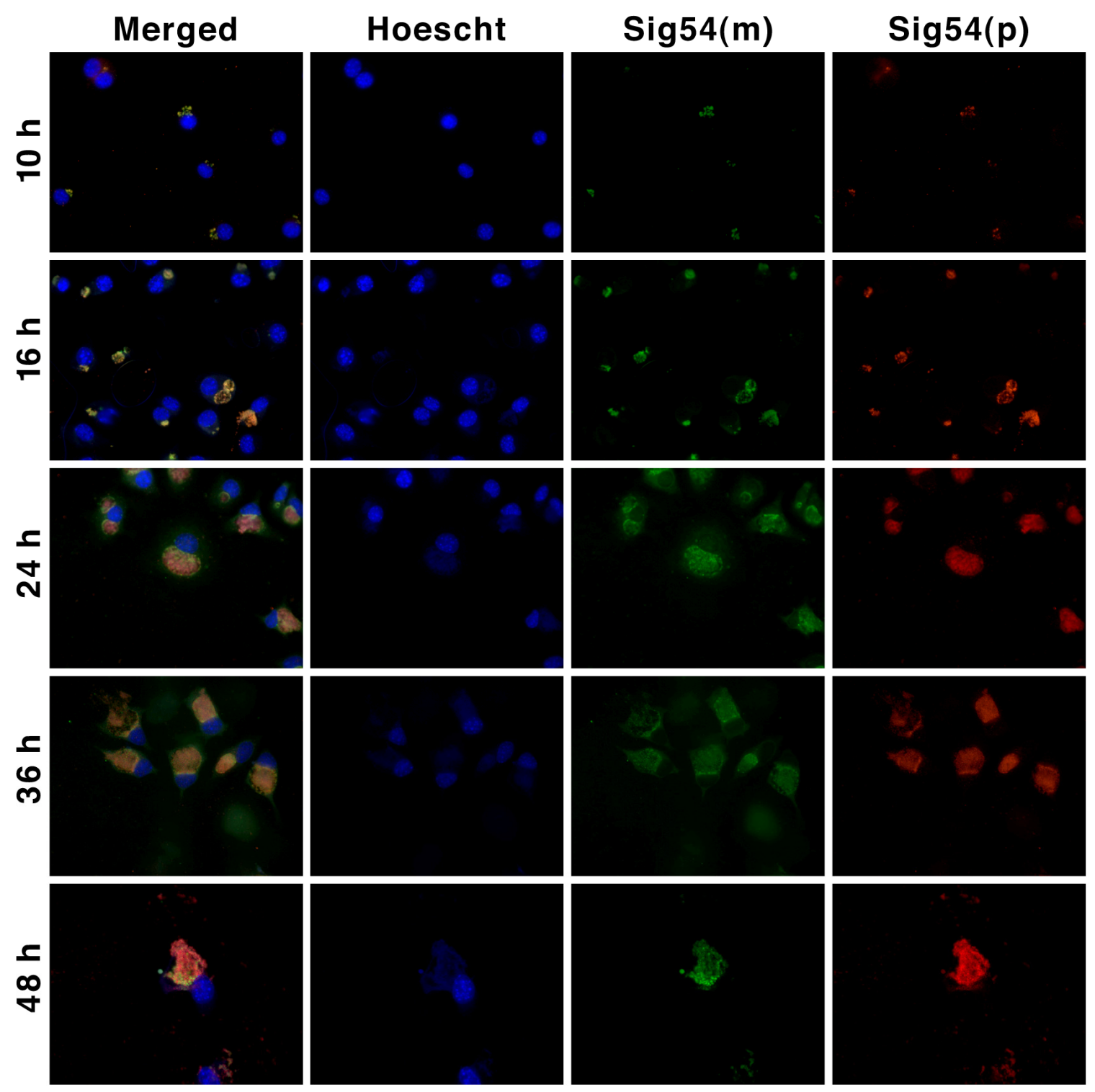

Figure 2-8. Polyclonal vs monoclonal IFA of sigma 54 in $C$. trachomatis-infected cells.

Infected cells were harvested at the times indicated and were fixed with $\mathrm{MeOH}$ for 48 hours. The cells were reacted with polyclonal sigma 54 and monoclonal sigma 54 antibodies. Hoescht was used to stain DNA. 


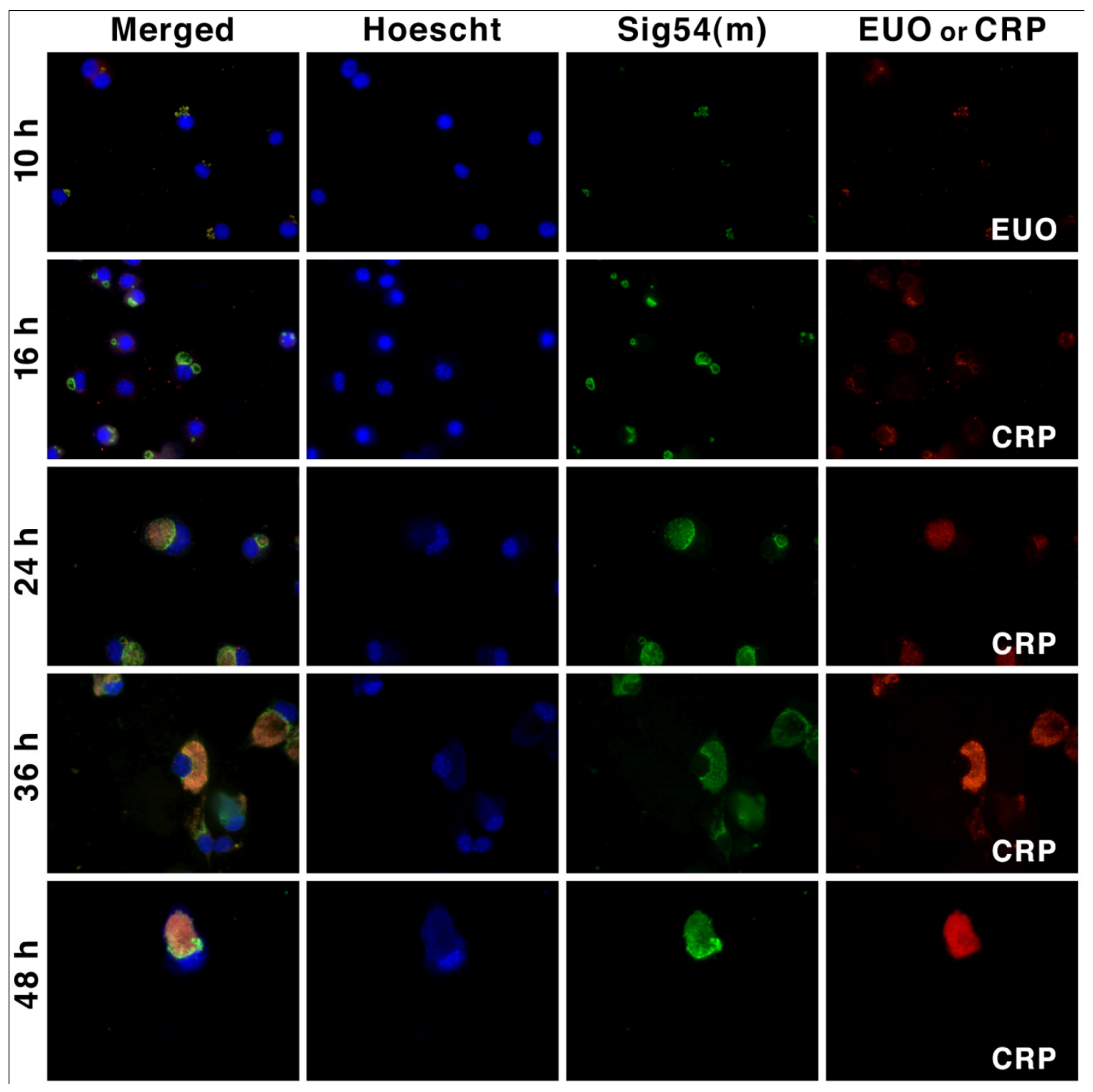

Figure 2-9. IFA of sigma 54 in $C$. trachomatis-infected cells during the developmental cycle using monoclonal antibody.

Infected cells were fixed with $\mathrm{MeOH}$ for 48 hours at the times indicated. The cells were reacted with monoclonal sigma 54 antibodies. As a counterstain, either polyclonal EUO (early) or polyclonal CRP (mid-cycle and late) antibodies were used. Hoescht was used to stain DNA. 
absent (Figure 2-10). Because of the small size of EBs (about 2 microns in diameter), it was not possible to resolve MOMP or sigma 54 staining to the OMC. One interpretation of these results is that the polyclonal antibody reacts with an antigen on the surface of EBs whereas the monoclonal antibody does not. Because of the cross-linked nature of the $\mathrm{OMC}$ in isolated EBs, it was not possible to ascertain whether either antibody reacted with an antigen in the cytoplasm of EBs. Western blot analysis was used to further examine the differences in antibody staining between the polyclonal serum and the monoclonal antibody. Initially, reaction to the Hatch lab recombinant His-tagged sigma 54 was examined by western blot to determine if the polyclonal and monoclonal antibodies detected denatured sigma 54. Both antibodies reacted identically, including reaction with a band that migrated around the $50 \mathrm{kDa}$ marker (Figure 2-11). Other bands, also seen on Coomassie stained gels (not shown), most likely degradation products, were observed with both the polyclonal serum and the monoclonal antibody. Next, reaction of the two antibody preparations with chlamydial extracts was examined (Figure 2-12). L cells were infected and chlamydiae were harvested at 16, 24, and 36, and 48 hpi. The sample size was adjusted so that reaction with MOMP was constant. Multiple banding was seen in the samples probed with the polyclonal serum, although the major band migrated below the $37 \mathrm{kDa}$ marker. The lower bands may be degradation products of the larger protein. The monoclonal antibody preparation reacted with a single band that migrated just above the $37 \mathrm{kDa}$ marker. In that the detection of sigma 54 in chlamydial extracts has not been reported in the literature, the relative migration rate of sigma 54 on gels is not known, thus it is not possible to conclude with certainty that the major polyclonal band or the single monoclonal band is sigma 54. However, the western analysis suggests that the two antibody preparations are not detecting the exact same antigen, although they may both be detecting some form of sigma 54 protein. Because the polyclonal serum IFA reaction pattern is similar to that of anti-MOMP (i.e., RBs look like "donuts"), the possibility of the polyclonal serum cross-reacting with MOMP was addressed. The variable antigenic regions of the MOMP are located on the surface of the OMC of chlamydiae. OMCs from EBs were prepared to assess if the antigen that is recognized by sigma 54 polyclonal serum is in the complex. This was performed by treating EBs with SDS, which solubilizes all proteins not present in the supra-molecular disulfide-bonded OMC. Once these complexes were isolated, they were allowed to attach to microscope slides and stained with sigma 54 polyclonal. Immunofluorescent assays revealed that sigma 54 polyclonal intensely stained the OMCs and overlapped the reaction with MOMP monoclonal antibody (Figure 2-13). This suggests that the sigma 54 polyclonal serum reacts with an antigen in the OMC, either the MOMP antigen or some unrelated $\mathrm{OMC}$ antigen.

To further assess the possible cross-reaction between the polyclonal sigma 54 serum and MOMP, purified EBs were treated with trypsin. MOMP stain was unaffected by trypsin treatment of the EBs (Figure 2-14), but sigma 54 polyclonal serum staining was nearly undetectable in the samples that were treated with trypsin. These experiments suggest that the sigma 54 polyclonal serum does not cross-react with the MOMP antigen and is fully accessible to trypsin in the $\mathrm{OMC}$ and, therefore, are not likely to be embedded in the outer membrane. 


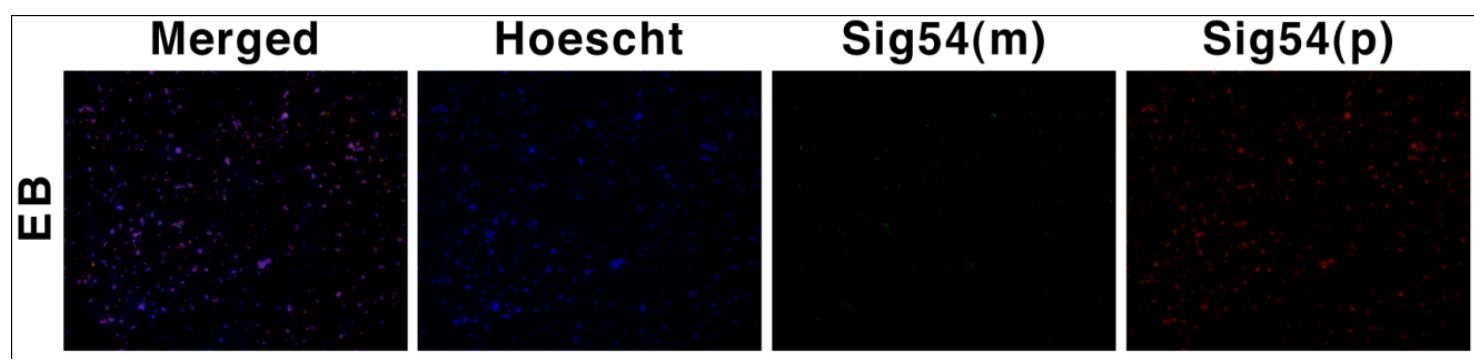

Figure 2-10. IFA of sigma 54 in purified $C$. trachomatis EBs.

EBs were harvested and purified at 48 hpi and fixed with $\mathrm{MeOH}$ for 48 hours. They were reacted with polyclonal sigma 54 and monoclonal sigma 54 antibodies. Hoescht was used to stain DNA. 


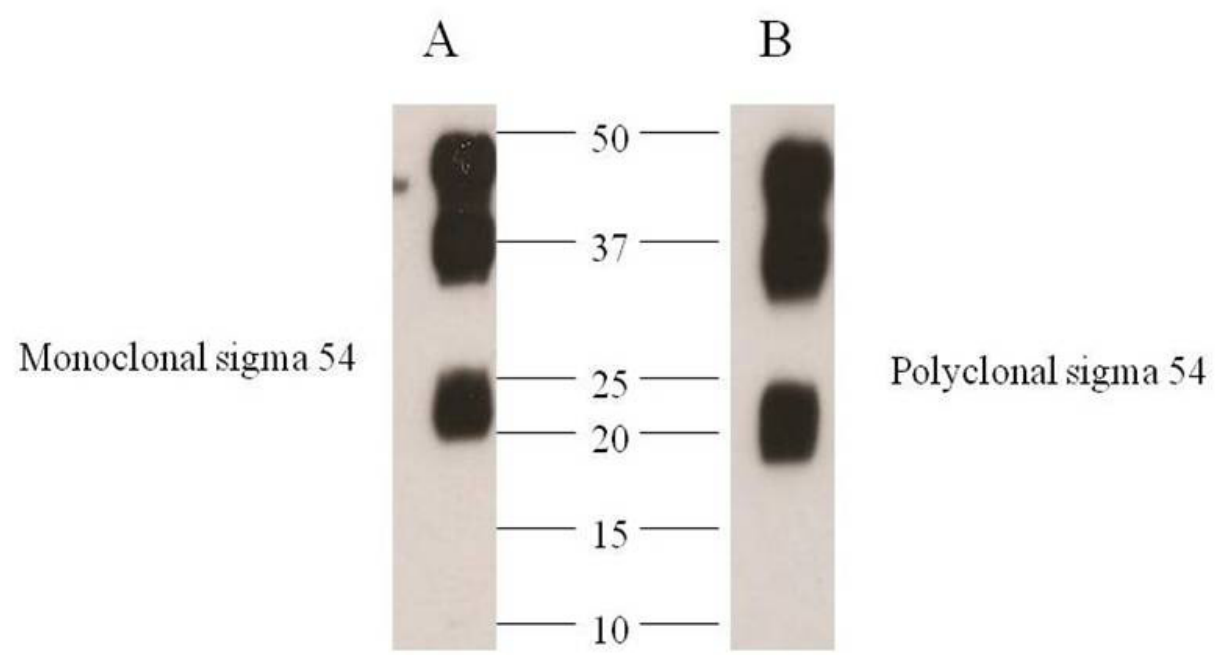

Figure 2-11. Western blot analysis of His-tagged sigma 54 protein.

Recombinant His-tagged sigma 54 was detected with (A) monoclonal antibodies and (B) polyclonal serum. 

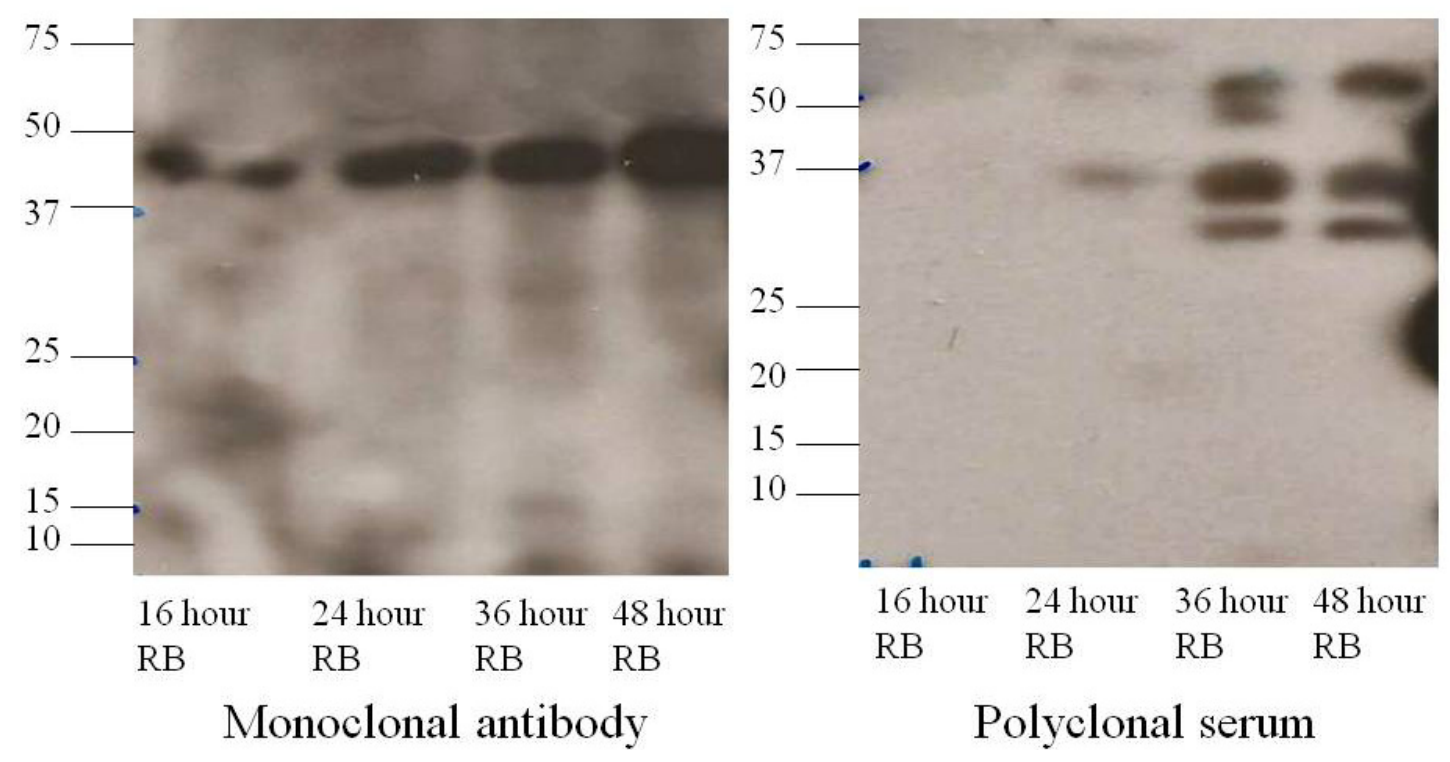

Figure 2-12. Western blot analysis of reticulate bodies using sigma 54 monoclonal antibodies and sigma 54 polyclonal serum.

Western blot analysis was performed in duplicate on gradient purified reticulated bodies isolated at 16, 24, 36, and 48 hours post-infection. One blot was probed with monoclonal antibodies to sigma 54 and the other blot was probed with polyclonal serum to sigma 54 . MOMP was used as a load control. 

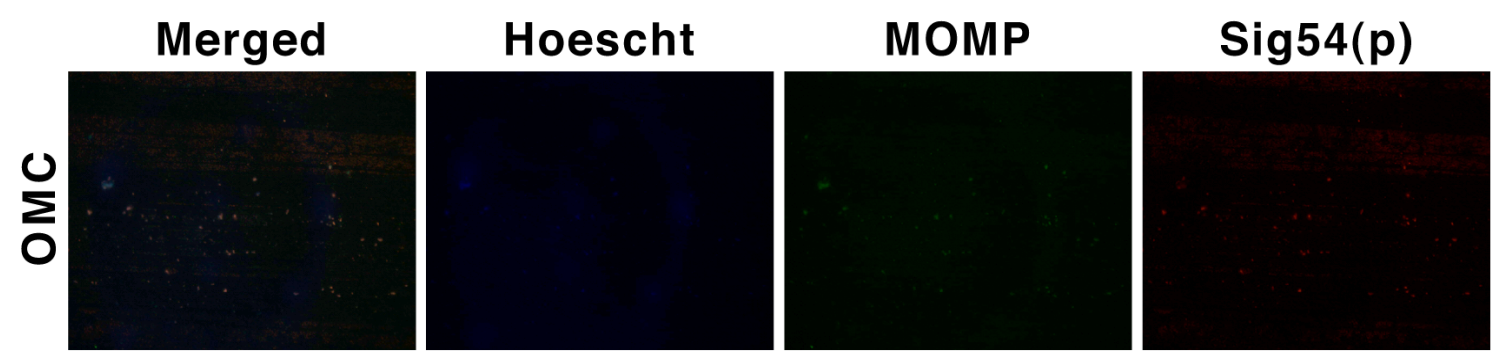

Figure 2-13. Immunofluorescence microscopy of $C$. trachomatis outer membrane complexes (OMCs).

SDS-OMCs were generated and fixed for 48 hours and were reacted with polyclonal sigma 54 antibodies and monoclonal MOMP antibodies. Hoescht stain was used as a negative control to determine the presence of contaminating EBs. 

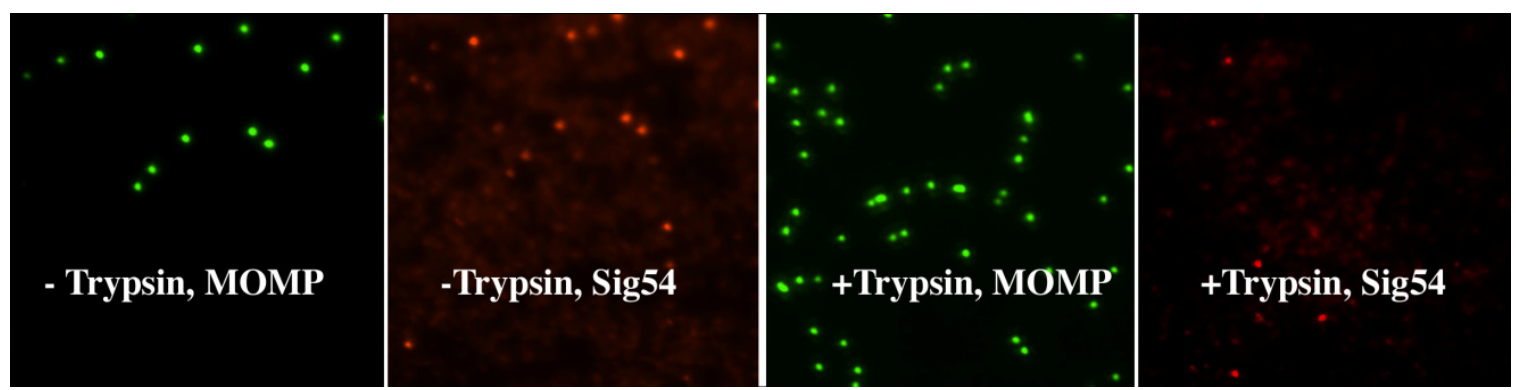

Figure 2-14. Immunofluorescence microscopy of $C$. trachomatis $\mathbf{E B s}$ treated with trypsin.

EBs were treated with trypsin and were reacted with polyclonal sigma 54 antibodies and monoclonal MOMP antibodies. 
To confirm this assessment, western blot analysis was performed on purified EBs (Figure 2-15). The samples were probed with either the MOMP antibody or the sigma 54 polyclonal serum. The MOMP antibody detected a band above $37 \mathrm{kDa}$ (the expected size of the MOMP), whereas the polyclonal sigma 54 antibody detected a band below $37 \mathrm{kDa}$, as seen previously in Figure 2-12, confirming that the polyclonal sigma 54 serum does not cross-react with MOMP. In summary, the sigma 54 serum detects a trypsin-sensitive antigen located in the OMC of EBs and this antigen also appears by IFA of infected cells to be present in the $\mathrm{OMC}$ of RBs by 16 to $24 \mathrm{hpi}$.

\section{DISCUSSION}

Reverse transcription polymerase chain reaction was used to monitor the expression of the sigma factors transcripts during the developmental cycle (Douglas and Hatch 2000). It is possible that these transcripts are not translated into protein; hence the rationale for examining sigma protein levels during the cycle.

In immunofluorescence assays, sigma 66 was seen to be expressed at the earliest time point ( 10 hours) examined and continued to be expressed through the later stages of the cycle. Western blot analysis showed sigma 66 being highly expressed at each time point examined, although time points before 16 hours could not be probed due to the difficulty in obtaining sufficient material to run on a western. Altogether, the results suggest that the sigma 66 protein is highly expressed throughout the developmental cycle. Sigma 66 is highly homologous to the major sigma factors sigma 70 of $E$. coli and sigma 43 of $B$. subtilis. Also, in vitro studies have shown sigma 66 regulating the expression of genes expressed throughout the developmental cycle, including housekeeping genes. Therefore it is believed to be the major sigma factor of chlamydia. This study supported this hypothesis. The alternative sigma factor, sigma 28 , was nearly absent at both early and very late time points when monitored via IFA; western analysis revealed similar findings: sigma 28 protein levels being highest during the mid-logarithmic phase with a gradual decline in expression at the latter stages of the infection cycle. In vitro studies have identified several genes that appear to be under the control of sigma 28 regulation. One of the most characterized of these genes is $h c t B$, the gene responsible for the production of one of the histone-like proteins. This gene is considered a late gene because it is not expressed at early time points ( 0 to $12 \mathrm{hpi}$ ), but transcripts can be detected as early as $16 \mathrm{hpi}$ (Fahr, Douglas et al. 1995; Belland, Zhong et al. 2003). It is possible that $h c t B$ is better considered an "early" late gene, and the synthesis of this histone-like protein plays a key role in the triggering of RB to EB conversion. Following this train of thought, sigma 28 , via its regulation of $h c t B$, may be "key" to the entry of RBs into the late phase of the cycle.

In preliminary studies not reported here, I (and others in the lab) confirmed by Northern blot analysis that sigma 28 is a late phase gene but the transcripts decline dramatically at the end of the cycle and are undetectable in EBs. This is in contrast to other late-phase genes, which appear to persist in EBs to the point that they can be detected up to 2 hours post infection in EBs in the process of converting to RBs 

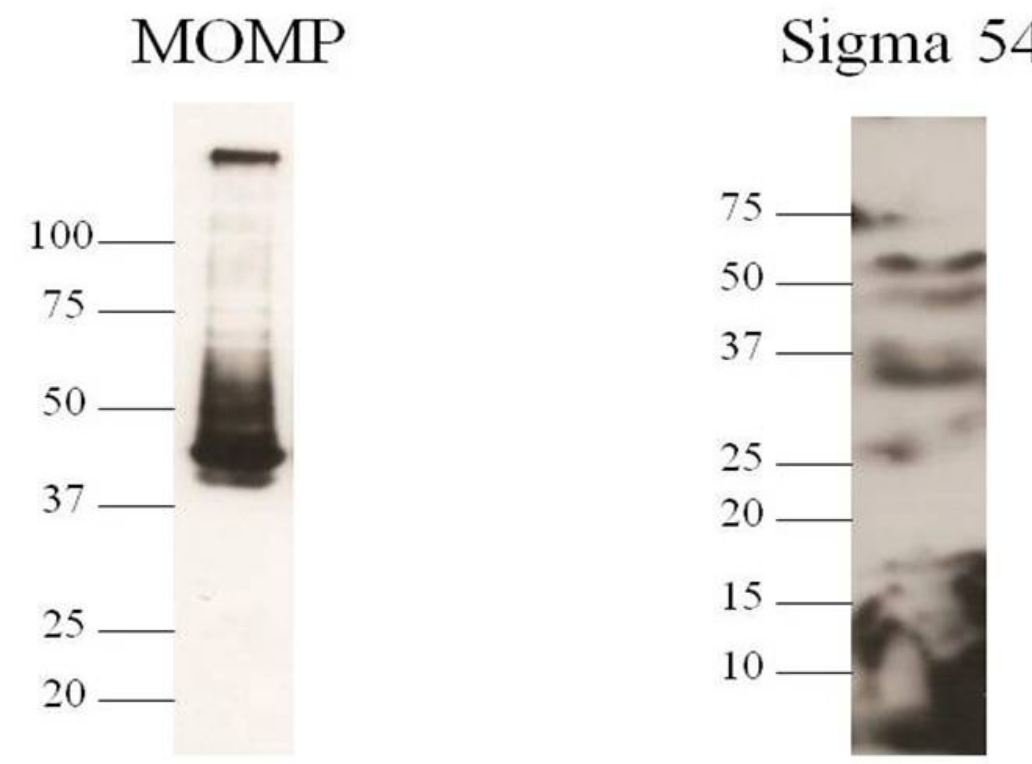

Figure 2-15. Western blot detection of MOMP and sigma 54 in EBs.

EBs were gradient purified and were probed with monoclonal MOMP antibodies or polyclonal sigma 54 serum. 
(Douglas and Hatch 2000; Belland, Zhong et al. 2003; Abdelrahman, Rose et al. 2011). These transcripts are not translated, but it is not known why this is so. In any event, the finding that sigma 28 transcription declines very late in the infection is consistent with the hypothesis that it plays a role very early in the RB to EB reorganization process rather than some terminal event. The temporal expression of sigma 54 was not completely settled by my studies because the rabbit polyclonal and mouse monoclonal sigma 54 antibodies appeared to identify different antigens. The polyclonal antibody recognized an antigen present in EBs (hence also present at very early time points post-infection) and at 10 hpi. Levels of sigma 54 antigen were highest late in the cycle when inclusions contained mostly EBs. Interestingly, the pattern of staining with the polyclonal changed from the 10 to the 24 hour samples. At $10 \mathrm{hpi}$, the pattern was somewhat punctate and at later times the antigen was associated with the OMC. One interpretation of this result is that at very early times (10 hours and earlier), the sigma 54 antigen present in infecting EBs undergoes a slow degradation in the cytoplasm and that newly synthesized protein during the logarithmic and late phases is associated with the OMC. The monoclonal antibody also detected antigen at 10 hours and later in the cycle but was not present in EBs, but the pattern of staining was consistent with a chlamydial cytoplasmic location. At very late times, the monoclonal stained primarily residual RBs still attached to the inclusion membrane. Whereas it is not clear what the nature of the polyclonal antigen is, the monoclonal most logically does detect cytoplasmic and presumably functional sigma 54, and that sigma 54 is found only in dividing RBs.

In silico studies predicted several possible targets for sigma 54 binding (Mathews and Timms 2000). Primer extension analysis identified two genes that may be targets for sigma 54 on the basis of location of possible bacterial sigma54 promoter sequences upstream from the 5' ends of the transcripts. Both genes are predicted to encode hypothetical proteins not closely related to other known bacterial proteins. The authors suggested that sigma 54 may be a late phage sigma factor on the basis of their RT-PCR analysis of sigma 54 RNA and the two target genes expression profiles. However, their data was not consistent with the transcriptome analysis of $C$. trachomatis (Belland, Nelson et al. 2003) and the studies of the Hatch lab (Douglas and Hatch 2000), which identified sigma 54 transcripts early in the logarithmic phase of growth. As stated above, my results are more consistent with sigma 54 being a mid-log phase sigma factor.

A significant problem when assessing the temporal function of sigma 54 is the insoluble nature of the full-sized recombinant protein, which precludes in vitro translation studies. Consequently, the target(s) and time of function of sigma 54 are not known.

An interesting observation in this study was the ability of the rabbit polyclonal sigma 54 serum to detect an antigen that stains intensely in the OMC of chlamydiae, particularly in very late phase inclusions. Two important questions arise from this observation: First, does the antibody recognize MOMP? My studies indicate that the antigen is not MOMP (Figure 2-14). Second, does the antibody recognize some form of sigma 54 protein or some totally unrelated chlamydial OMC antigen? Whatever the antigen may be, it is unlikely to be the CRP, which is the only OMC antigen present in high amounts in the OMC other than the MOMP because CRP is a late protein and the 
polyclonal antibody reacts well with mid-log phase RBs (Figure 2-9). Also, whatever the antigen is, it presumably was present in the recombinant sigma 54 preparation used to generate the antigen. Was the antigen an E. coli contaminant with the same migration rate on the gel as recombinant sigma 54? This seems unlikely in that no known E. coli 60 $\mathrm{kDa}$ protein (the size of the recombinant) cross-react with any known chlamydial OMC proteins. Was the antibody in the rabbit pre-bleed serum? No (Thomas Hatch, Annemarie Douglas, personal communication 1997). Is the antigen the same as the poorly characterized "Rocky peptidoglycan" antigen (Brown and Rockey 2000). This is possible, but rendered unlikely by the intense staining in EBs that appear to lack peptidoglyan and the susceptiblitly of the antigen to trypsin (Figure 2-14). Altogether, the identity of the antigen remains unsettled, but the simplest conclusion is that it recognizes some form of sigma 54 in that it was raised against recombinant sigma 54. If the antigen is some form of sigma 54, what is it doing in the OMC? Does sigma 54 have a dual function; an initiator of transcription and a structural role?

The scope of this study was to monitor the protein expression profiles of the sigma factors during the developmental cycle in order to gain insight into their possible role in control temporal events in the cycle. My study succeeded in confirming that sigma 66 protein, like its transcripts, is found throughout the cycle. The study also supported the working hypothesis that at least one sigma factor may function in the regulation of stage-specific genes. Specifically, the peak level of sigma 28 protein at the early-late phase suggests that it may play a critical role in the transition of RBs to EBs. Although the evidence was not entirely clear, my results suggest that the cytoplasmic sigma 54 is a mid-log sigma factor and not a late sigma factor, as previously postulated (Mathews and Timms 2000).

Future studies beyond the scope of my studies, which might confirm my speculations and more clearly define the function of the alternative sigma factors, will be discussed in the final chapter of this dissertation. 


\section{CHAPTER 3. IN VITRO IDENTIFICATION OF EUO BINDING SITES USING CHROMATIN IMMUNOPRECIPITATION TO MICROARRAY CHIP ANALYSIS}

\section{INTRODUCTION}

All chlamydial species have a unique developmental cycle. The cycle begins with chlamydia in an extracellular, metabolically-inactive form termed the elementary body (EB). Once the EB attaches and is internalized into the target host cell, it begins to differentiate into the non-infectious, metabolically-active reticulate body (RB). After several rounds of growth and division the RB switches back to the EB form when it exits the cell and begins another round of infection. The mechanism that is responsible for the switch between the EB and RB is unknown. In an effort to identify genes that may be responsible for the initial conversion of the $\mathrm{EB}$ to $\mathrm{RB}$, a scheme was developed (Wichlan and Hatch 1993) to identify genes that are expressed early in the developmental cycle. One of the genes identified was upstream of the gltx gene and was named the early expression upstream open reading frame of the gltx gene (euo). This gene contains a helix-turn-helix motif, a characteristic of DNA-binding proteins, and was later seen to be capable of binding DNA. Because of its time of expression and its ability to bind DNA, it was postulated to be a possible regulator of genetic expression.

Attempts were made to identify the DNA-binding sites of EUO and derive a consensus binding sequence. Investigators (Zhang, Howe et al. 2000) used the SELEX method (Systematic Evolution of Ligands by Exponentially Enrichment) to address which regions of the genome was capable of being bound by EUO. This protocol entailed incubating recombinant EUO with enzyme-digested DNA ligated into a Bluescript vector allowing the protein to bind the DNA. The protein/DNA mixture was allowed to pass through a membranous filter. Unbound DNA was capable of passing through the filter whereas the DNA that was bound by the EUO protein passage through the filter was abrogated. The protein-bound DNA was isolated from the filter, cloned, and subjected to another round of enrichment. This procedure was repeated nine times in an effort to select out the strongest binding sites for EUO. Several clones were highly selected after the nine rounds of enrichment. These selected EUO binding sites were located within open reading frames (ORFs), which did not agree in principle of EUO being a transcriptional regulator. A weak consensus binding sequence was derived from the selected clones, although multiple mismatches had to be allowed to identify the clones that were selected.

This study was another attempt to identify EUO binding sites in vitro. A microarray chip has been developed using the Chlamydia trachomatis serovar D genome. The chip contains both the ORFs and the intergenic regions of the genome. This study will use this microarray chip to perform the Chromatin Immunoprecipitation to Microarray Chip protocol (ChIP-to-Chip). In this procedure, chlamydia-infected cells are formaldehyde-crosslinked in an effort to preserve the EUO/DNA interaction. EUO is then immunoprecipitated along with its bound DNA. The DNA is isolated, labeled, and 
allowed to hybridize with the microarray chip. The major difference between my proposed study and that of Zhang et al. (2000) is that I will examine the extent of binding of EUO to small fragments (25-mers) in the entire chlamydial genome rather than use a selection technique to find the most well-bound fragments. In short, my approach was thought to be more global.

The working hypothesis of this study is that the microarray readout will reveal both strong and weak EUO binding sites within the chlamydial genome that will allow the derivation of a consensus and prediction of stage-specific EUO. For example, strong binding to early genes would suggest transcriptional activation, whereas binding to late genes would suggest suppression of transcription. Another goal is to derive a consensus sequence for EUO binding and identify the genes that may be regulated by EUO.

\section{MATERIALS AND METHODS}

\section{Growth and Isolation of Chlamydia Trachomatis Serovar D}

HeLa cells were grown in $150 \mathrm{~cm}^{2}$ vented cap flasks with DMEM. After reaching $75 \%$ confluency (20,000 cells) the cells were infected with C. trachomatis serovar D and allowed to incubate at 37 degrees under $4 \% \mathrm{CO} 2$. At the time of harvest, the infected HeLa cells are treated with a 1\% solution of trypsin (Gibco) to detach the cells from the flask without lysing. The cells were then collected and were subjected to Renocal gradient purification with the similar protocol as outlined in Chapter 2.

\section{Preparation of Recombinant His-Tagged EUO}

E. coli BL21(DE3)pLysE was transformed with the pET-28(+) vector that contained the entire EUO gene ligated into the EcoRI site. E. coli was then grown in 200 $\mathrm{mLs}$ of Luria-broth overnight and spun down 1,000 rpm to form a pellet. The pellet was then dissolved in a $\%$ SDS solution to release the overexpressed insoluble inclusion body. The His-tagged EUO was then purified by nickel-agarose affinity chromatography.

\section{Electrophoresis Mobility Shift Assay}

The cysteine-rich operon (omcAB) upstream DNA was radioactively labeled with fifty $\mu \mathrm{Ci}{ }^{32} \mathrm{P}$-dATP following the instructions of the random primed DNA labeling kit from Roche. After labeling, the radioactive probe was allowed to incubate with either recombinant EUO or RB extracts in $20 \mu \mathrm{L}$ of DNA binding solution $(25 \mathrm{mM}$ Tris- $\mathrm{HCl}$, $70 \mathrm{mM} \mathrm{KCl}, 20 \mathrm{mM}$ EDTA, $50 \mu \mathrm{g} / \mathrm{ml} \mathrm{BSA}, 3 \mathrm{mM} \mathrm{CaCl}_{2}, 1 \mathrm{mM}$ dithiothreitol, $7 \%$ glycerol) for an incubation period of one hour. For supershift experiments, anti-EUO serum was added at a dilution of 1:100. Protein-bound DNA was separated from free 
probe by running the samples on a 5\% native polyacrylamide gel. After electrophoresis the gel was dessicated and was exposed to autoradiograph film (Kodak).

\section{Immunoprecipitation of EUO from RB and Recombinant EUO/Co- immunoprecipitated DNA Isolation}

Reticulate bodies isolated 18 hours post-infection (hpi) were crosslinked by treatment with formaldehyde at a $1 \%$ final concentration. The crosslinked RBs were lysed with RIPA buffer (300 mM NaCl, $50 \mathrm{mM}$ Tris, 1\% NP-40, 0.5\% Deoxycholate, $0.1 \%$ SDS) and allowed to incubate with a 1:100 dilution of anti-EUO serum for one hour. For the recombinant EUO immunoprecipitations, his-tagged EUO and sonicated genomic chlamydial DNA were brought up to a $300 \mu \mathrm{Ls}$ volume to ensure mixing. The samples were washed three times in RIPA buffer. If the samples were to be subjected to western blot analysis then the immunoprecipitation samples were suspended in $30 \mu \mathrm{Ls}$ of digest mix. If the samples were to be subjected to DNA isolation, they were treated with $100 \mu \mathrm{Ls}$ of ChIP elution buffer (1\% SDS and $20 \mathrm{mM}$ EDTA) and were allowed to incubate overnight at $65^{\circ} \mathrm{C}$ to reverse the formaldehyde crosslinks. After overnight incubation, the DNA from these samples were isolated via phenol/chloroform extraction. The DNA was washed twice with $95 \%$ ethanol and suspended in $10 \mu \mathrm{Ls}$ of deionized water.

\section{ChIP-to-Chip Analysis}

Isolated EUO co-imunoprecipitated DNA was amplified using the WGA2 whole genome amplification kit (Sigma). After amplification the DNA was labeled with either Cy3 or Cy5 and allowed to hybridize to the MPAUT (Multi-Pathogen Array University of Tennessee) array. cDNA was generated from total genomic RNA and was used as a positive DNA control.

\section{RESULTS}

\section{Identification of EUO in Chlamydia Trachomatis Serovar D}

The microarray chip used for these experiments was generated using the Chlamydia trachomatis serovar D genome; therefore, I had to use Chlamydia trachomatis serovar D to perform the ChIP-to-chip procedure. HeLa cells were infected and chlamydia was harvested at 18 hpi. Eighteen hours post-infection was chosen because obtaining sufficient amounts of chlamydia (and EUO) to run on a gel before this time point proved difficult. Also, probing extracts later than eighteen hours resulted in a decrease in the amounts of EUO observed compared to the eighteen hour time point (data not shown). These samples were subjected to western blot analysis and probed for EUO. 
The lab did not possess antibodies to Chlamydia trachomatis serovar D EUO, so I used serum that was generated against Chlamydia trachomatis serovar L2 EUO. BLAST searches revealed that the sequences of serovar D EUO and serovar L2 EUO was highly homologous (99\% identity). As a control, uninfected HeLa cells were run on the gel in an effort to identify any non-specific antibody cross-reaction with the host cell. I was able to detect a single band in the eighteen hpi sample (Figure 3-1). This band migrated below the $20 \mathrm{kDa}$ marker which, is below the expected molecular size of $27 \mathrm{kDa}$ for the EUO protein. We assumed this band was EUO due to the fact that it was the only band observed when the sample was probed with the anti-EUO serum. Also, there was no cross-reaction of the anti-EUO serum with the uninfected HeLa cell sample, indicating that this reaction was Chlamydia specific. The difference in the expected migration rate of EUO and the observed band was attributed to a difference in migration rates of EUO in different species of Chlamydia. In our lab, when we probe for EUO in C. psittaci extracts, EUO appears to migrate at the $25 \mathrm{kDa}$ marker; probing C. trachomatis $\mathrm{L} 2$ extracts, EUO migrates to the $20 \mathrm{kDa}$ marker. Therefore, we were confident that the protein we were detecting in the $C$. trachomatis serovar D extracts was EUO.

\section{Chlamydia Trachomatis Serovar D Binds DNA}

I verified that EUO was present in $C$. trachomatis serovar D extracts and that I was capable of detecting it with the $C$. trachomatis serovar L2 serum. The next step was to test itsability to bind DNA. In our lab, we have verified the ability of EUO to bind DNA using electrophoresis mobility shift assays (EMSA); thus, I decided to check the ability of serovar D to bind DNA via this protocol. Chlamydiae were grown in HeLa cells and harvested at $18 \mathrm{hpi}$, the time point that EUO appeared to be most abundant in $C$. trachomatis serovar D extracts. The DNA probe used to test if EUO was capable of binding DNA was the upstream region of the CRP promoter region (Figure 3-2). Past experiments have shown that EUO from both $C$. trachomatis serovar L2 and C. psittaci are capable of binding this 267 base pair region of DNA; therefore, I used this region of DNA to examine the ability of $C$. trachomatis serovar D EUO to bind DNA. The probe was generated using forward and reverse primers to PCR-amplify this region of DNA, using genomic DNA as a template. A sample containing the CRP promoter region and recombinant His-tagged EUO was used as a control. In the lane containing His-tagged recombinant EUO and DNA there was shift in migration of the DNA probe (Figure 3-3). A similar shift was seen in the lane containing 18 hpi chlamydial extracts and the CRP probe. The addition of anti-EUO serum caused a super-shift of the probe, indicating that the shift was due to EUO binding the probe and not a non-specific protein binding the DNA. I concluded that EUO from C. trachomatis serovar D was able to bind DNA; or more specifically, to bind to the CRP promoter region of DNA.

\section{Immunoprecipitation of EUO from C. Trachomatis Serovar D Extracts}

Since I was able to detect EUO in C. trachomatis serovar D extracts and was able to demonstrate that EUO was capable of binding DNA, the next step was to immunoprecipitate EUO from chlamydial extracts. Chlamydia-infected HeLa cells were 


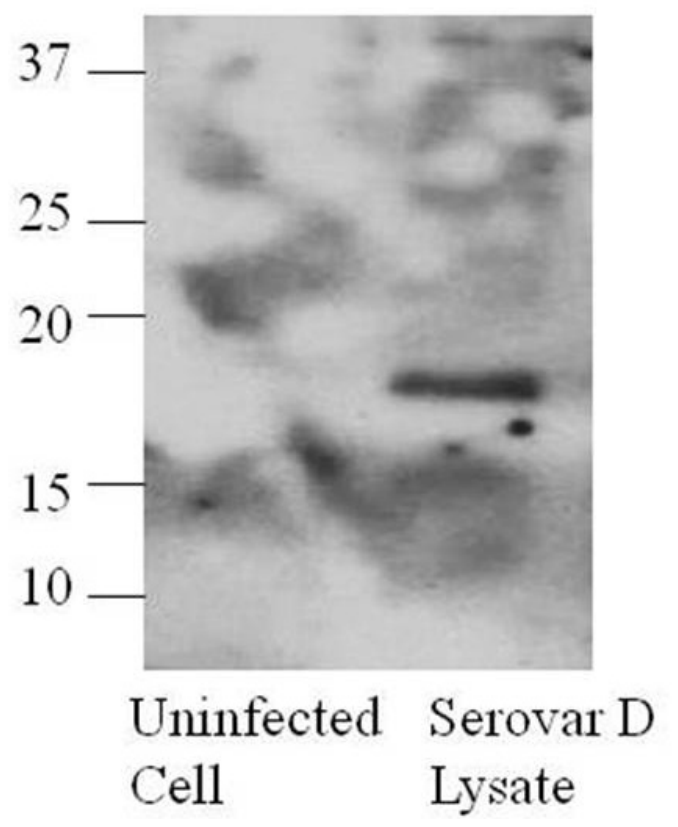

Figure 3-1. Detection of EUO in Chlamydia trachomatis serovar D extracts.

Western blot analysis was performed on uninfected HeLa cells (lane 1) and 18 hpi RBs. Antibodies generated against L2 recombinant his-tagged EUO was used to for detection. 


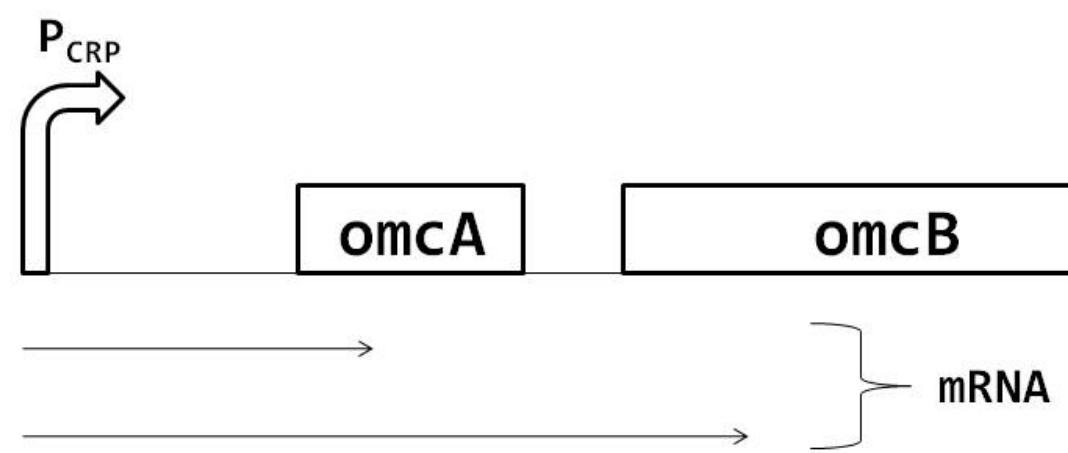

GAAGTTTGTTTTGTTTGTAGGCAAACTTATCTCTTTGAATCCGAGCTGT

TTATTATTTTATTTTGTTTGCTTTGATTTGCTAATTACCTGTTATTAGA

CGATTTGTTTTAAAAAACAATTGATATAATTTTTATTTTATA $-35$

$-10$

\section{TTGTCTATGAGGGCTAGTTTCT}

$+1$

Figure 3-2. Cysteine-rich protein (CRP) operon.

The CRP operon encodes two genes: $o m c A$ and $o m c B$, which are produced late in the developmental cycle. Transcription of the operon occurs both as a monocistronic message, which only codes for $o m c A$, and a bicistronic message, which codes for both the $o m c A$ and $o m c B$ gene. 


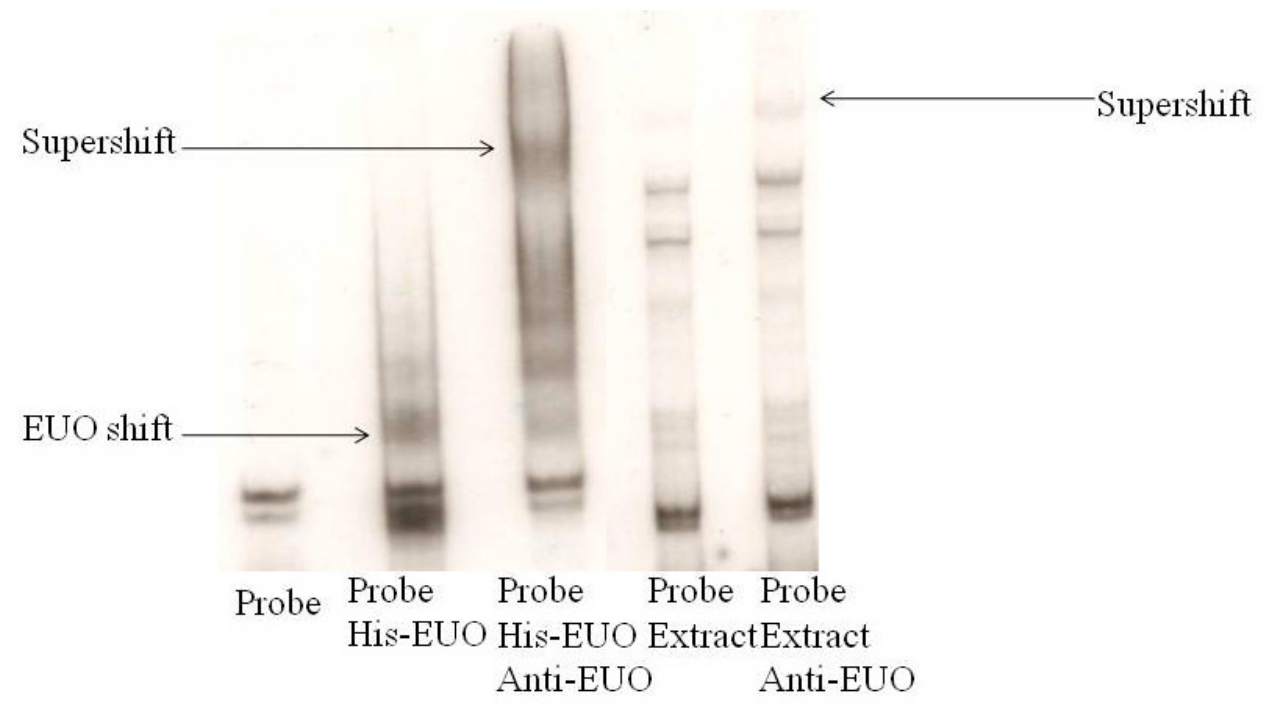

Figure 3-3. Electrophoretic mobility shift assay of the cysteine-rich protein operon $(-154$ to +14$)$.

Both recombinant His-tagged EUO and native EUO from extracts were used to shift gelpurified crp operon. Lanes: 1, free probe; 2 , probe + his-EUO; 3 , probe + his-EUO + anti-EUO; 4 , probe $+18 \mathrm{~h}$ serovar D extracts; 5 , probe $+18 \mathrm{~h}$ serovar D extracts + antiEUO. 
harvested and the RBs were isolated and purified at 18 hpi. RBs were formaldehyde cross-linked in an effort to preserve the physiological interactions between EUO and DNA. By doing this, the DNA that is immunoprecipitated with EUO should be the DNA that EUO would bind in the context of an infection and not be caused by random binding of EUO to non-specific DNA during lysis. After the cross-linking process, the RBs were allowed to incubate with anti-EUO serum and protein A-sepharose beads. Following incubation, the immunoprecipitation samples were washed three times with RIPA buffer which contains detergents. The purpose of these washes was to eliminate any nonspecific binding. After the washes, the antibody/protein complex was isolated and run on a gel and subjected to western blot analysis (Figure 3-4). A band was present in the experimental lane (immunoprecipitation using extract and anti-EUO serum) and was absent in the control lane (immunoprecipitation using extract and prebleed serum), indicating that this band was specific to the anti-EUO serum. This band, however, migrated just below the $25 \mathrm{kDa}$ marker, which was higher than expected. When $18 \mathrm{hpi}$ chlamydial extracts were probed, a minor band was detected at the $25 \mathrm{kDa}$ marker but a major band was detected below the $20 \mathrm{kDa}$ marker. This lower band was believed to be EUO in previous experiments. Repeated immunoprecipitation experiments resulted in the immunoprecipitation of the $25 \mathrm{kDa}$ band and not the $20 \mathrm{kDa}$ band. I surmised the 20 $\mathrm{kDa}$ band to be a degradation product of EUO that was incapable of being immunoprecipitated by the EUO antibodies. Since I was able to immunoprecipitate fulllength EUO, I proceeded with the attempt to isolate the co-immunoprecipitated DNA.

\section{Isolation of Co-Immunoprecipitated DNA from EUO Immunoprecipitations}

With the successful immunoprecipitation of EUO protein from Chlamydia trachomatis serovar D extracts, the next step was to isolate the co-immunoprecipitated DNA. Once isolated, this DNA would then be labeled and allowed to hybridize to the microarray chip to reveal EUO binding sites within the genome. After EUO was immunoprecipitated, the sample was treated with ChIP elution buffer, which functions in disrupting the protein A-sepharose beads/antibody/protein/DNA bonds. The samples were then heated at sixty-five degrees overnight to reverse the formaldehyde crosslinks. Once these bonds were broken, the samples were treated with a phenol/chloroform solution in order to isolate the DNA from the proteins. Next the isolated DNA was loaded and run on a $1 \%$ agarose gel. No DNA was observed (data not shown). It was possible that the amount of the DNA being co-immunoprecipitated with EUO was not sufficient to be visualized on an agarose gel. I attempted to amplify the DNA that coimmunoprecipitated with EUO in hopes of being able to visualize the DNA on the agarose gel. After the immunoprecipitation of EUO and the isolation of the bound DNA, the DNA was subjected to amplification using the Whole Genome Amplification (WGA2) kit (Sigma Aldrich). The samples were then run on a 1\% agarose gel. The amplification step did result in visualization of DNA on the gel (data not shown). It seemed that DNA was not being co-immunoprecipitated with the EUO protein. Another possibility relates to the amount of EUO being immunoprecipitated. Since EUO is a minor protein, it can be conceived that the amounts of EUO being immunoprecipitated 


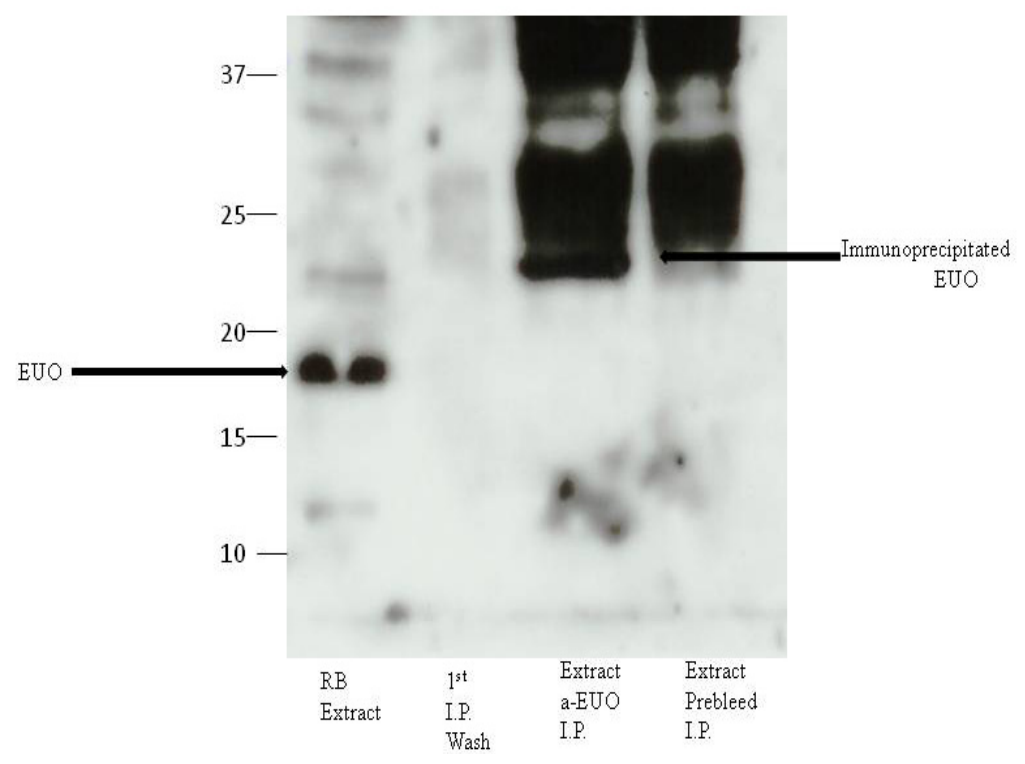

Figure 3-4. Immunoprecipitation of EUO from Chlamydia trachomatis serovar D extracts.

Anti-EUO antibodies were used to immunoprecipitate EUO from 18 hpi extracts. Lanes: 1, 18h serovar D extracts: 2, 1st RIPA buffer wash supernatant: 3, 18h serovar D extracts + anti-EUO: 4, 18h serovar D extracts + Prebleed serum. 
was not sufficient in bringing down enough DNA to efficiently amplify; thereby resulting in the ability to visualize DNA on an agarose gel.

\section{Immunoprecipitation of Recombinant His-Tagged EUO and Isolation of Co- Immunoprecipitated DNA}

The ChIP-to-Chip assay is an in vivo protocol that requires the immunoprecipitation of a DNA-binding protein from live cells in hopes of coimmunoprecipitating the DNA that it binds. I was not able to proceed with the ChIP-toChip experiment under our previous conditions using chlamydial extracts; therefore, I decided to switch to an in vitro approach. I used recombinant EUO and isolated chlamydia genomic DNA to perform the immunoprecipitatons. This would circumvent the obstacle of not having enough EUO present to successfully co-immunoprecipitate DNA, as seen in the aforementioned in vivo ChIP-to-Chip experiment.

This in vitro approach required the immunoprecipitation of recombinant Histagged EUO. Attempts to formaldehyde crosslink the recombinant His-tagged EUO and the genomic DNA resulted in aggregates of EUO that could not be resolved on a polyacrylamide gel (data not shown). I opted to omit the formaldehyde cross-linking step in an effort to successfully immunoprecipitate recombinant His-tagged EUO, although the omission of the formaldehyde cross-linking step could result in the disruption of the recombinant EUO/DNA interaction which would lead to the inability to coimmunoprecipitate DNA. With the omission of the cross-linking step, I was able to successfully immunoprecipitate recombinant His-tagged EUO (Figure 3-5).

Immunoprecipitation of EUO was dependent on anti-EUO serum because using prebleed serum did not result in the immunoprecipitation of recombinant EUO. The next step in the ChIP-to-Chip protocol was the isolation of the DNA from the immunoprecipitated protein. I was able to immunoprecipitate recombinant His-tagged EUO with the antiEUO serum, but because I omitted the formaldehyde cross-linking step, I wanted to verify that during the immunoprecipitation procedure that the recombinant protein/DNA interaction was not disrupted. If I was able to show that DNA was coimmunoprecipitated with recombinant EUO, I could assume that the interaction between the two was not disrupted.

The immunoprecipitation was performed with the same conditions as before. No DNA was observed (Figure 3-6). I attempted the WGA amplification protocol and DNA was visualized in the lane containing DNA isolated from the EUO immunoprecipitation. DNA was not observed in the control lane that used prebleed serum to immunoprecipitate the recombinant His-tagged EUO. This would indicate that recombinant EUO/DNA interaction was not disrupted with the omission of the cross-linking step and that the presence of co-immunoprecipitated DNA was dependent on recombinant EUO being immunoprecipitated. 


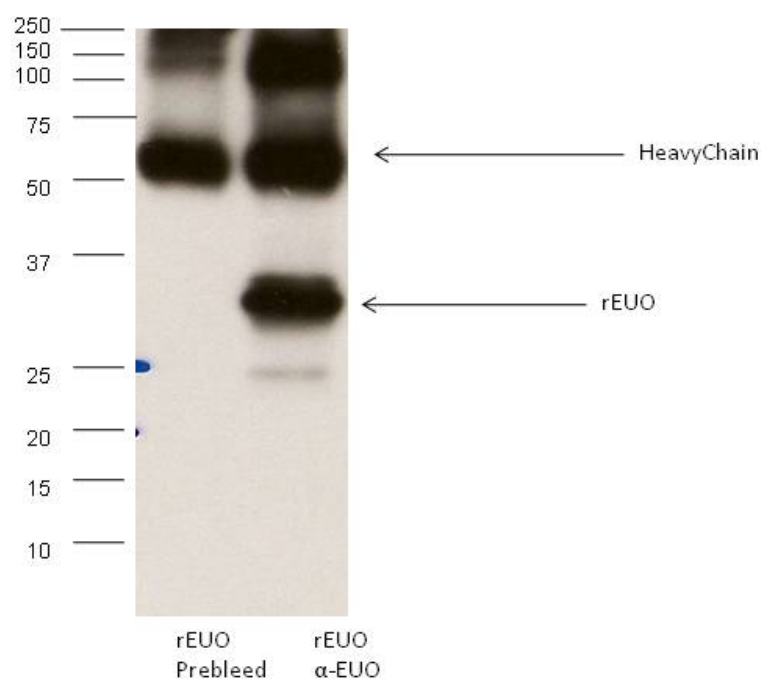

Figure 3-5. Immunoprecipitation of recombinant EUO.

Recombinant His-tagged EUO was immunoprecipitated with anti-EUO in RIPA buffer. Lane 1, his-tagged EUO + prebleed serum; Lane 2, his-tagged EUO + anti-EUO. 


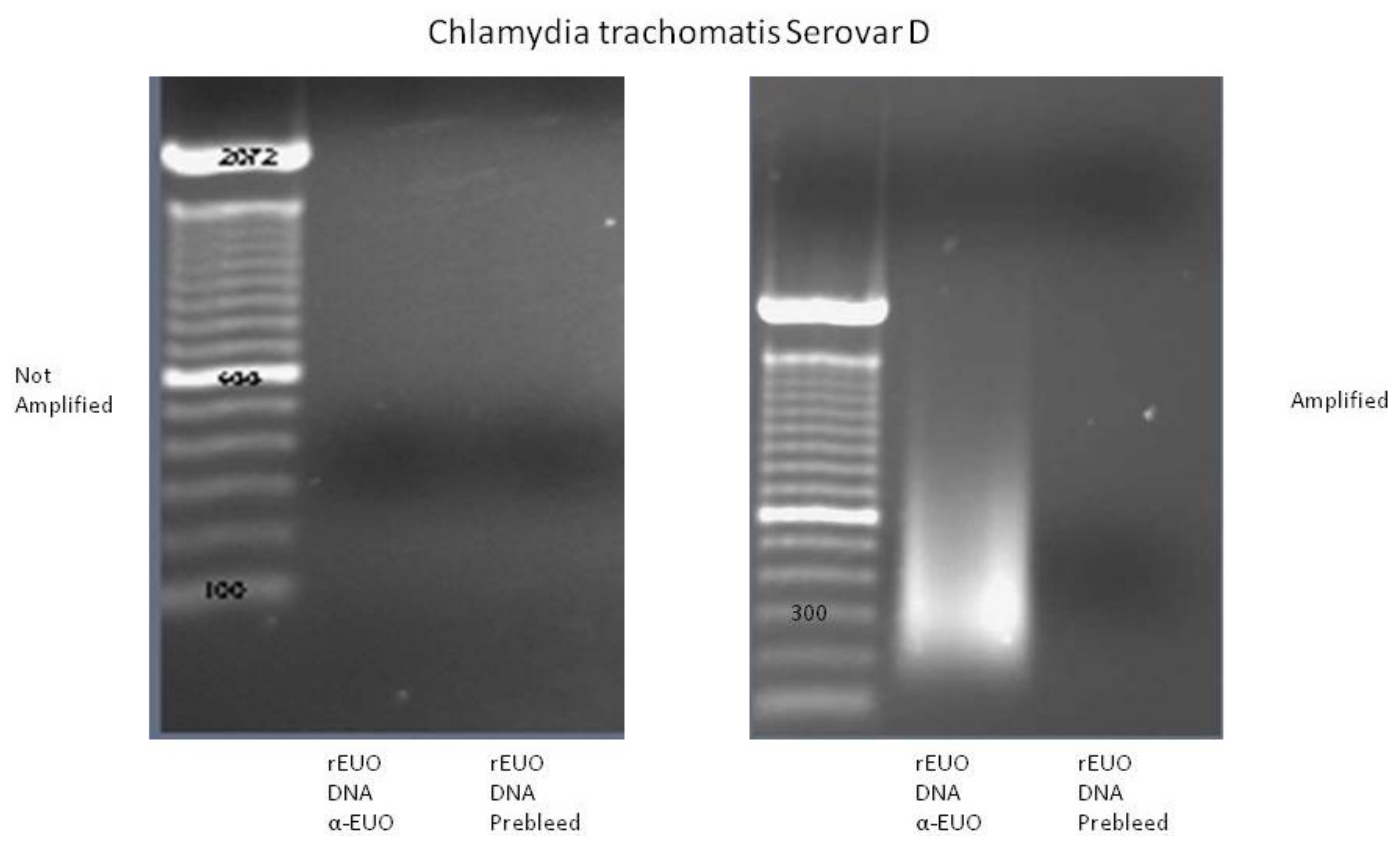

Figure 3-6. Isolation and amplification of EUO co-immunoprecipitated DNA.

His-tagged EUO was immunoprecipitated and the EUO-bound DNA was isolated and run on a $1 \%$ agarose gel. Panel 1 shows the migration of co-immunoprecipitated DNA: lane 1, anti-EUO immunoprecipitated His-tagged EUO; lane 2, prebleed immunoprecipitated his-tagged EUO. A DNA ladder is shown at the left side. Panel 2 shows the migration of amplified co-immunoprecipitated DNA; lane 1, anti-EUO immunoprecipitated histagged EUO: lane 2, prebleed immunoprecipitated his-tagged EUO. A DNA ladder is shown at the left side. 


\section{EUO Binds Multiple Regions throughout the Chlamydial Genome}

Once the DNA was isolated from the recombinant His-tagged EUO immunoprecipitation and amplified, it was then labeled and then allowed to hybridize to the MPAUT-1 microarray chip. The microarray layout is a linear representation of the chlamydial genome. Each probe (dot on the microarray representing a 25-base segment of DNA) is arranged in a tip-to-tail fashion with the beginning of the genome starting in row one/column one of the layout progressing through the genome with the last sequences of the genome being represented at the bottom right corner of the layout. The microarray contains both the intergenic regions and the open reading frames (ORF) of the genome. The intergenic regions have a complimentary dot in the opposite strand, whereas the ORFs are represented as a single dot without a complimentary dot in the opposite strand. The cryptic plasmid was represented as a succession of unpaired dots located at the end of the microarray chip. The microarray layout revealed EUO bound to multiple regions of the genome (Figure 3-7). A red dot represented a probe that bound strongly with EUO whereas a blue dot denoted a probe that weakly associated with the EUO protein. Both red dots and blue dots were scattered on the microarray layout. There were no single regions of the chlamydial genome that seemed to be highly concentrated with sequences that were specific for EUO binding. This experiment was performed three times with different preparations of DNA and recombinant His-tagged EUO, and similar results were seen in each experiment, suggesting that binding was specific rather than due to random background binding.

\section{EUO Does Not Preferentially Bind AT-Rich Sequences}

Since there was no obvious region of the genome that seemed to be specific for EUO binding, I decided to focus my attention on analyzing the sequences of DNA that were being bound by EUO. Zhang et al. demonstrated that EUO preferentially bound AT-rich sequences (1998); therefore, I wanted to evaluate if there was a preference for AT-content for EUO binding in my studies. Each probe on the microarray was a representation of a 25-base pair sequence; so I inspected the probes that bound EUO with high affinity (Table 3-1) and compared their AT-content with the AT-content of the probes that weakly associated with EUO (Table 3-2). The AT-content of the probes with the highest hybridization values (fifty-four percent) was lower than the AT-content of the probes with the lowest hybridization values (sixty-six percent), which was unexpected because previous studies have shown that EUO preferentially binds AT-rich sequences.

Analyzing individual probes did not reveal a preference for AT-content in EUO binding. During the immunoprecipitations, EUO was binding fragments of DNA larger than 25 base pairs (between 200-700 base pairs as visualized on the gel); therefore, I decided to scan the microarray for regions containing consecutive probes with high hybridizing values to examine if these larger regions would reveal AT-content being a determinant for EUO binding. Regions containing four or more consecutive probes with high hybridizing values were selected and the AT-content was analyzed. The region with the most consecutive probes with the highest hybridization values was the intergenic 


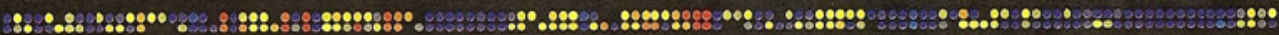

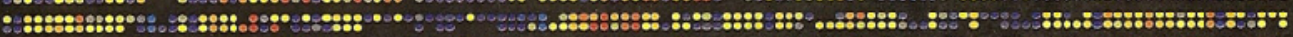

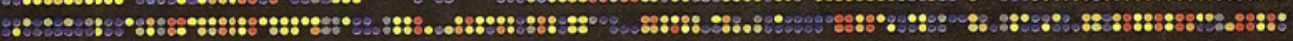

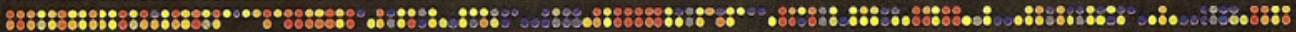

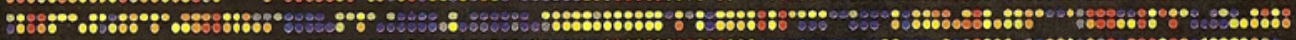

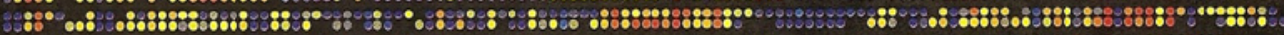

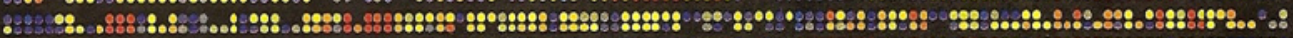
25. 8.

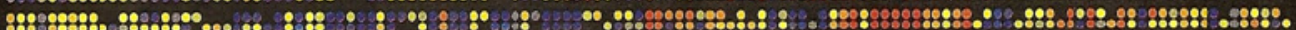

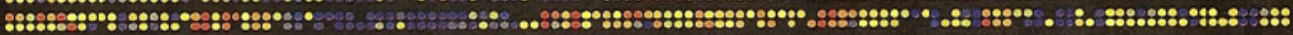

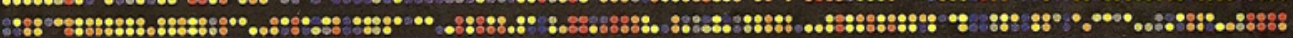
88888 . ระ8.

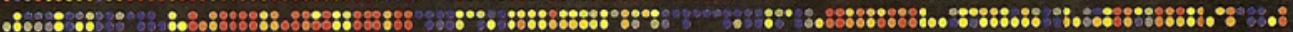
9898 989

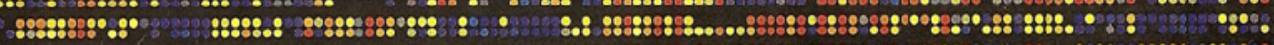
8. • . E.5.

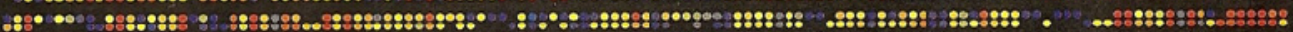
¿.98.8

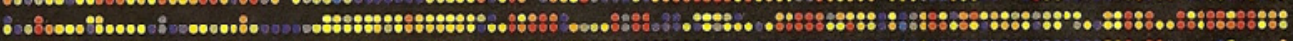

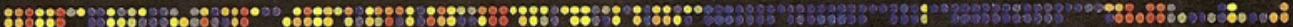

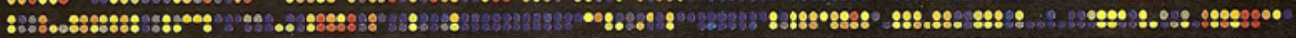

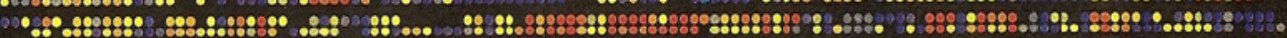

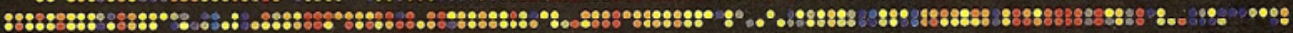

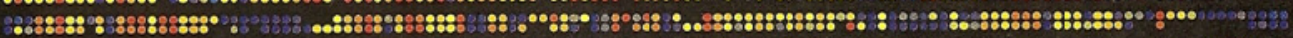

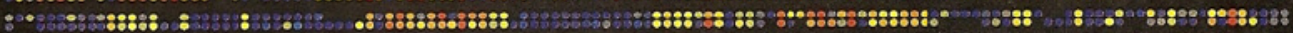

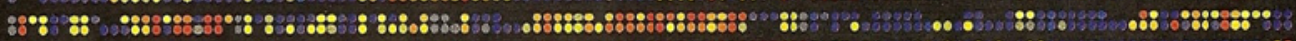

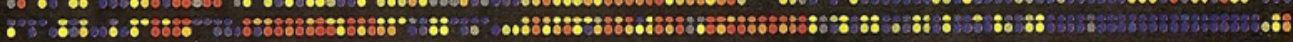

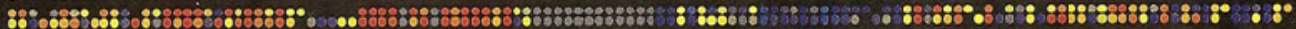
•

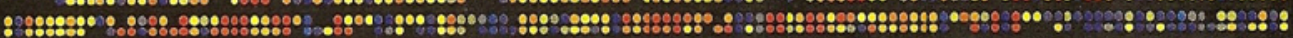
2月. 8в

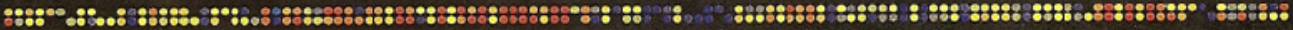
8.8.88: \&

Figure 3-7. ChIP-to-Chip layout of EUO co-immunoprecipitated DNA hybridized to the $C$. trachomatis serovar $\mathrm{D}$ genome.

Degree of binding is indicated from high affinity (red) to low affinity (blue). Gray indicates probes that weakly hybridized with the genomic DNA control. 
Table 3-1. Probes with the strongest hybridization to EUO coimmunoprecipitated DNA.

\begin{tabular}{lcc}
\hline $\begin{array}{c}\text { IGRCT } \\
\text { Number }\end{array}$ & Sequence & $\begin{array}{c}\text { Hybridization } \\
\text { Value }\end{array}$ \\
\hline 663.56 & GCAAACACACCCAGAGACAGTCCAA & 29245 \\
266.03 & TCAAAAACAACCAAAAACCAACTAT & 16469 \\
873.22 & TTAACCGTAGCCAAGCACACAGAGA & 16044 \\
343.12 & ACAATCCCCGGTTCTTGGCGTT & 15860 \\
224.49 & AAAATCGAACAGACAACACAATGG & 15603 \\
744.25 & ACAGAGCGGTACAAGGAGATGCAA & 15223 \\
829.02 & CTCCAACCCCAAACCTCACCACTCT & 15011 \\
544.27 & ATGCGGCGTATTGTGATGGATAA & 14653 \\
343.03 & GAACGCACAAGAGACCGGGATTGT & 14564 \\
080.07 & AGAGGGATAACGGCAAGGTTGTA & 14129 \\
342.10 & GACGAAACACCACTAACAAAGATT & 13886 \\
809.35 & GTCGCTGAGCAACGAAACACAAGGA & 13856 \\
740.16 & AAAAGGAAGCGAAGATGCAAAAAGA & 13615 \\
150.12 & GGTTCAGAAAGTGAACGTTAGAG & 13383 \\
544.04 & ACGAGACCACCATGTACACCAAAA & 13154 \\
552.17 & GCAATCCGAACAAAAGCTCTTGAGA & 13035 \\
873.25 & TCTCTGTGTGCTTGGTACGGTTAA & 12693 \\
450.36 & GCGGTGATGAGCAAGGAAAAAGAC & 12685 \\
809.01 & TCCTTGTGTTCGTTGCTCAGCGAC & 12497 \\
150.09 & CACCGTGTCTAAAGTATGCGACCAG & 12175 \\
\hline
\end{tabular}

Note: IGRCT $=$ Intergenic region Chlamydia trachomatis. 
Table 3-2. Probes with the weakest hybridizations to EUO coimmunoprecipitated DNA.

\begin{tabular}{lcc}
\hline $\begin{array}{c}\text { IGRCT } \\
\text { Number }\end{array}$ & Sequence & $\begin{array}{c}\text { Hybridization } \\
\text { Value }\end{array}$ \\
\hline 446.09 & GCCCCCCTCCGGAATTCCGGAGGG & 15.3 \\
593.1 .1 & CTGAAGAGAAAAATTCTCTCTCCC & 15.0 \\
033.06 & ATTTAAGAGCTATCTAATATAGT & 13.2 \\
629.03 & AACCTTTGCAAACAAGGTTATG & 13.2 \\
423.09 & CGATAAAAATTTATCGATATAGA & 13.0 \\
033.05 & ACTAATATTAGATAGCTTCTTAAT & 11.5 \\
326.2 .9 & TCTTTGTAGACCTAAAGGTCTAGT & 11.5 \\
273.13 & CAATCATAATCGGGGAGGGAAAA & 11.4 \\
643.09 & CAGACCCAATATCATTGGTCCCGG & 11.2 \\
020.03 & AAAGAGGTTTTAATAGACCTCT & 9.6 \\
604.08 & TCTATTAAAGAATTTTAACAGAA & 9.6 \\
498.17 & GGTAAGTCTTTGAAAAGACTCAGC & 9.3 \\
423.02 & TCTATATCGATAAAAATTTATCG & 9.0 \\
643.63 & AGGAAATGTCAAGGACATTGCTC & 8.9 \\
619.02 & GCTCGCAGGTATATATCTGCGAGCT & 7.6 \\
805.15 & TGTTTTTTCTATCGAAAAAAATCA & 7.3 \\
873.07 & TTTTGTTTATTCAAACAAAAGGT & 7.3 \\
750.17 & AAATAGAGTTTAAGAACTCTAGGGA & 7.2 \\
413.03 & AATTAGGCAAGGATACCTTGCCTA & 5.0 \\
805.01 & TGATTTTTTCGATAGAAAAAACA & 1.5 \\
\hline
\end{tabular}

Note: IGRCT $=$ Intergenic region Chlamydia trachomatis. 
region IGRCT 343 (Figure 3-8). This region was fifty-eight percent AT-rich in content. For comparison, I scanned the microarray for a region of consecutive probes with low hybridizing values; intergenic region IGRCT 446 (not shown) contained seven consecutive probes with low hybridizing values and this region was seventy-three percent AT-rich. These results would suggest that there were factors, other than AT-content, that influenced EUO binding to these sites.

\section{Analysis of Selected Intergenic Regions and Open Reading Frames}

As stated above, the intergenic region that exhibited the strongest hybridization with the EUO co-immunoprecipitated DNA was intergenic region 343. This region was upstream of a gene that encodes for a protein from the $\mathrm{O}$-sialoglycoprotein endopeptidase family. The expression profile of the O-sialoglycoprotein endopeptidase in chlamydiae has yet to be studied; therefore, I could not compare the expression of EUO with the expression of this protein. The intergenic region with the highest hybridization values that preceeded a gene with a well-characterized expression profile was the intergenic region preceding the late transcription unit $\mathrm{B}(\mathrm{ltu} B)$ gene. A previous study has shown that $l t u B$ transcripts are first expressed at $24 \mathrm{hpi}$, coinciding with the decrease in expression of the EUO protein; therefore, it is possible that EUO may act as a negative regulator of the $l t u B$ gene expression. Further inspection of intergenic regions also revealed a non-late gene possibly being regulated by EUO. Intergenic region 232, which is upstream of the $i n c B$ gene, also exhibited strong hybridizing with the EUO coimmunoprecipitated DNA. The incB gene encodes for a protein that is produced and translocated to the inclusion membrane. The $i n c B$ protein, in contrast with the late expression of the $l t u B$ gene, is produced as early as 2 hpi (Shaw, Dooley et al. 2000). It would seem, according to the intergenic data, that EUO may have a dual role in genetic regulation; repressing the expression of late genes and activating the expression of early genes.

Analyzing the ORFs proved more difficult than analyzing the intergenic region. The intergenic region sequences are represented on the microarray chip arranged in a tipto-tail fashion. Therefore, there was complete sequence coverage of the intergenic regions and the value of each probe that bound EUO can be determined. The ORFs are represented as a single probe comprised of eleven 25-mers. The hybridizing values for the ORFs are calculated as an average of the eleven 25-mers and the exact value of each 25-mer within the ORF could not be determined. Because I could not determine which individual 25-mer was responsible with the hybridization with EUO coimmunoprecipitated DNA, I could not analyze the sequences within the ORFs. Rather, my analysis was restricted to examining the rankings of the ORFs based on their hybridizing values. I grouped both the top twenty ORFs with the highest hybridization values (Table 3-3) and the bottom twenty ORFs (Table 3-4) with the lowest hybridization values and evaluated them to see if EUO preferred binding to a particular subset of genes. The top twenty ORFs contained a varied array of genes with different functions. The gene $r s b V 2$ is a possible transcription regulator. Glycogen synthase, $g \lg A$, produces an enzyme that participates in the gluconeogensis. HctA encodes for a histone-like protein that binds DNA and has a role in the condensation of chlamydial chromatin. Four of the 


\begin{tabular}{|c|c|c|c|}
\hline Probe 1 & CTTAATACGTAATTGGTTTTATTTA & TATTTAATAACGCGCGCCTAGCTC & Probe 2 \\
\hline Probe 14 & GAATTATGCATTAACCAAAATAAAT & ATAAATTATTGCGCGCGGATCGAG & Probe 13 \\
\hline Probe 3 & GAACGCACAAGAGACCGGGGATTGT & AACACCACTTTTATTTAGGCTTCAA & Probe 4 \\
\hline Probe 12 & CTTGCGTGTTCTCTGGCCCCTAACA & TTGTGGTGAAAATAAATCCGAAGTT & Probe 11 \\
\hline Probe 5 & GAGATACTTAAGTTGTATAACCTTC & CTCGATCCGCGCGCAATAATTTATT & Probe 6 \\
\hline Probe 10 & СТCTATGAATTCAACATATTGGAAG & GAGCTAGGCGCGCGTTATTAAATAA & Probe 9 \\
\hline Probe 7 & AGTAAACAAAACTCTTGAAAAGTTG & Top & \\
\hline Probe 8 & TCATTTGTTTTGAGAACTTTTCAAC & Bottom & \\
\hline
\end{tabular}

\section{Figure 3-8. Intergenic region 343.}

Probe alignment of the intergenic region with the highest hybridization values. Letters in red indicate probes with high hybridization values and letters in black indicate probes with low hybridization values. 
Table 3-3. Ranking of the ORFs with the highest hybridization values to EUO co-immunoprecipitated DNA.

\begin{tabular}{llcc}
\hline $\begin{array}{l}\text { Gene } \\
\text { Name }\end{array}$ & \multicolumn{1}{c}{ Gene Description } & $\begin{array}{c}\text { Hybridization } \\
\text { Value }\end{array}$ & $\begin{array}{c}\text { Time of } \\
\text { Expression }\end{array}$ \\
\hline ltu A & late transcription unit A & 5675 & $16 \mathrm{~h}$ \\
hctA & histone-like developmental protein & 4369 & $24 \mathrm{~h}$ \\
r17 & 17/112 ribosomal protein & 4232 & $3 \mathrm{~h}$ \\
rs21 & S21 ribosomal protein & 4179 & $3 \mathrm{~h}$ \\
y fhC & cytosine deaminase & 4086 & $3 \mathrm{~h}$ \\
r113 & L13 ribosomal protein & 4057 & $3 \mathrm{~h}$ \\
ltuB & late transcription unit B & 4039 & $24 \mathrm{~h}$ \\
rsbV2 & sigma factor regulator & 4009 & $16 \mathrm{~h}$ \\
nusG & transcription termination protein & 3794 & $8 \mathrm{~h}$ \\
dsbG & possible disulfide bond chaperone & 3634 & $8 \mathrm{~h}$ \\
yebL & solute-binding protein & 3309 & $8 \mathrm{~h}$ \\
yjfH & rRNA methylase & 3279 & $3 \mathrm{~h}$ \\
oppB 1 & oligopeptide transport system & 3212 & \\
& permease protein & & $3 \mathrm{~h}$ \\
rnc & ribonuclease III & 3201 & $8 \mathrm{~h}$ \\
incB & inclusion membrane protein B & 3063 & $3 \mathrm{~h}$ \\
inc A & CHLPS inclusion membrane protein & 3053 & \\
& A & & $16 \mathrm{~h}$ \\
exbB & polysaccharide transporter & 3000 & $3 \mathrm{~h}$ \\
glgA & glycogen synthase & 2968 & $16 \mathrm{~h}$ \\
pgs 1 & CDP-diacylglycerol-glycerol-3- & 2913 & \\
& phosphate 3-phosphatidyltransferase & & $3 \mathrm{~h}$ \\
rs20 & s20 ribosomal protein & 2912 & $3 \mathrm{~h}$ \\
\hline
\end{tabular}


Table 3-4. Ranking of the ORFs with the lowest hybridization values to EUO coimmunoprecipitated DNA.

\begin{tabular}{llcc}
\hline $\begin{array}{l}\text { Gene } \\
\text { Name }\end{array}$ & \multicolumn{1}{c}{ Gene Description } & $\begin{array}{c}\text { Hybridization } \\
\text { Value }\end{array}$ & $\begin{array}{c}\text { Time of } \\
\text { Expression }\end{array}$ \\
\hline fliF & flagellar M-ring protein & 152 & $16 \mathrm{~h}$ \\
yojL & hypothetical protein & 147 & $8 \mathrm{~h}$ \\
rs14 & S14 ribosomal protein & 146 & $16 \mathrm{~h}$ \\
ybcL & hypothetical protein & 145 & $24 \mathrm{~h}$ \\
pnp & polyribonucleotide nucleotidyltransferase & 132 & $8 \mathrm{~h}$ \\
gspE & general secretion pathway protein E & 131 & $3 \mathrm{~h}$ \\
groEL_3 & mitochondrial HSP60 chaperonin & 121 & $16 \mathrm{~h}$ \\
yscJ & yop translocation J & 121 & $8 \mathrm{~h}$ \\
pmpI & putative outer membrane protein I & 117 & $3 \mathrm{~h}$ \\
yprS & hypothetical protein & 117 & $8 \mathrm{~h}$ \\
hemZ & ferrochelatase & 115 & $8 \mathrm{~h}$ \\
plsX & FA/phospholipid synthesis protein & 102 & $8 \mathrm{~h}$ \\
murA & UDP-N-acetylglucosamine transferase & 91 & $3 \mathrm{~h}$ \\
lcrD & low calcium response locus protein D & 91 & $8 \mathrm{~h}$ \\
maf & maf-type protein & 90 & $8 \mathrm{~h}$ \\
rl32 & 132 ribosomal protein & 79 & $8 \mathrm{~h}$ \\
gpdA & glycerol-3-P dehydrogenase & 79 & $16 \mathrm{~h}$ \\
lipA & lipoate synthetase & 75 & $16 \mathrm{~h}$ \\
pyrH & UMP kinase & 70 & $8 \mathrm{~h}$ \\
fliI & flagellum-specific ATP synthase & 57 & $16 \mathrm{~h}$ \\
\hline
\end{tabular}


top twenty ORFs were genes that encoded for ribosomal proteins but two ribosomal proteins were also present in the bottom twenty ORFs. There did not appear to be a particular subset of genes that was being bound by the EUO protein. I next examined the time of expression of the ORFs that EUO bound, comparing the time of expression of the top twenty hybridizing ORFs with the time of expression of the bottom twenty hybridizing ORFs. The time of expression between the two was very similar. They both contained genes that were expressed as early as $3 \mathrm{hpi}$, as well as genes that were expressed as late as 24 hpi. This observation, similar to the observation of the intergenic regions, would suggest that EUO may have a dual role in transcription regulation in Chlamydia.

\section{DISCUSSION}

The binding of recombinant EUO (rEUO) to DNA was first reported in 1998 by Zhang et al. The ability of rEUO to bind DNA was used to select clones that preferentially bound to the protein. Although that study resulted in the identification of several preferential binding sites for EUO which led to the derivation of a consensus sequence; the consensus was weak and required multiple mismatches to identify other selected clones. It also did not address the in vivo significance of the consensus and the binding sites remains obscure, in part because the binding sites were all located within ORFs and the ORFs had no obvious connection among them, such as function or time of expression. Therefore, I re-examined the in vitro binding of rEUO to DNA, using a more global approach via ChIP-to-Chip analysis to identify short fragments of genomic DNA bound to rEUO.

The microarray approach proved to be much more difficult than anticipated. $C$. trachomatis serovar D DNA was required and, although this serotype is easier to propagate than many others, yields are low; ranging between 10 to 1000 times lower than serovar L and $C$. psittaci. To remedy this short-coming, I developed a DNA amplification procedure based on the relatively new and novel WGA amplification kit.

Initially, the microarray results were highly promising. Three totally independent experiments yielded an impressive replication of results with nearly all hot-spots and cold spots remaining the same, suggesting that binding of rEUO to DNA was "real", at least at the in vitro level. However, interpretation of the results was confounding. For one thing, hot-spot intergenic microarray probes (25-mers) to which rEUO-precipitated DNA bound were no more AT-rich than cold spots, a serious difference from various previous results (Zhang, Douglas et al. 1998; Zhang, Howe et al. 2000). Furthermore, the hot spots failed to yield a consensus. Various other analyses of the intergenic regions also failed to yield a meaningful conclusion. rEUO consistently bound strongly to some ORFs (and weakly to others), but these results also defied interpretation. No correlation could be made among the ORFs, either function or time of expression. Another discouraging result was the failure to find an overlap among the Zhang et al. (1998) sequences and my results. 
The only conclusions I draw from my results are (1) rEUO appears to consistently bind to some genomic DNA fragments in vitro better than others; (2) rEUO binds to many sequences in vitro; (3) strong binding in vitro is not restricted to intergenic regions; (4) there is no evidence for rEUO preference for AT-rich sequence, despite multiple previous contrary findings; (5) binding to a consensus sequence was not evident; (6) the significance of my results, albeit reproducible, remains obscure. In short, the working hypothesis that this study would reveal specific binding sites that might allow the prediction of EUO function and derivation of a consensus binding site was not supported.

It is possible that in vitro binding of rEUO to DNA is totally irrelevant to in vivo events. This would be usual in that most, if not all other known bacterial DNA binding factors bind well, and specifically, in vitro to DNA elements identified by in vivo approaches such as analysis of mutants. It is possible that EUO requires a cofactor (protein or ion) for tight specific binding or that the conformation of native DNA in RBs engenders more specific binding, which would explain the relatively non-specific binding of rEUO to DNA in vitro. With this in mind, I undertook the in vivo studies described in the next chapter.

An interesting side observation made in the course of my studies was the size discrepancy between native EUO detected by western blot in RB extracts of $C$. trachcomatis serovar D (smaller size) and EUO detected by western blot in EUO immunoprecipitated from RB extracts (larger size). The smaller fragment may be a degradation product of the larger one. Presumably the larger fragment is a minor component that was concentrated by the precipitation procedure whereas the dominant smaller fragment was not precipitable. Whether or not both fragments bind to DNA is not known, but failure of the small fragment to bind may be part of the regulation of EUO function. 


\section{CHAPTER 4. IN VIVO IDENTIFICATION OF EUO BINDING SITES USING CHROMATIN IMMUNOPRECIPITATION AND CLONING ANALYSIS}

\section{INTRODUCTION}

EUO is produced early in the developmental cycle of Chlamydia. The role of EUO during the developmental cycle is not known although it is believed to be a possible transcriptional regulator due to its time of expression and its DNA-binding capabilities. The expression of the euo gene is one of the most highly expressed, if not the most highly expressed gene, within the first few hours of infection (Wichlan and Hatch 1993). However, protein levels peak during the logarithmic stage of growth when reticulate body (RB) forms are rapidly replicating. EUO begins to decrease in expression early in the late stage of the cycle, at the time of RB/EB transition. Ouellette et al. (2006) also demonstrated that EUO transcripts are upregulated during IFN- $\gamma$ mediated persistence. EUO has been shown to bind multiple regions throughout the genome (Zhang et al., 2000 and Chapter 3), but attempts to identify specific binding sites have been met with limited success. The SELEX method performed by Zhang et al. (2000) and the ChIP-to-Chip method performed in Chapter 3 were both in vitro methods; consequently, both procedures neglected auxiliary factors, such as accessory proteins or DNA structure that may influence EUO's binding specificity.

In this chapter, I describe the development of a ChIP-Cloning protocol for identifying genomic fragments bound to native EUO in intact RBs isolated at 16 hours post-infection (hpi) and in infected cell extracts at 4 hpi. In an attempt to ensure the EUO-DNA interactions were not disrupted during manipulations, the RBs and the extracts were treated with formaldehyde to cross-link EUO protein to DNA. The immunoprecipitated DNA was cloned and sequenced and BLAST searches were then performed to identify EUO-bound genomic fragments. The working hypothesis is similar to that in Chapter 3; in vivo analysis of DNA fragments bound by native EUO will identify strong and weak binding sites that will lead to a prediction of EUO function.

The genes and fragments identified in this study were randomly distributed throughout the genome and did not fall into any stage-specific or functional category. They also did not overlap with the genes identified in the in vitro ChIP-to-Chip assay from Chapter 3, suggesting that sequence alone is not the only determinate in EUO binding and that other factors contribute in EUO binding specificity. Further studies are necessary to elucidate the function of EUO. However, my studies in Chapter 3 and this chapter suggest that EUO binding does not strictly regulate early gene expression but rather may facilitate, in some unknown fashion, transcription in general during the early and logarithmic phases of growth. 


\section{MATERIALS AND METHODS}

\section{Growth and Isolation of Chlamydia Psittaci}

L929 mouse fibroblasts were grown in spinner culture with Medium 199. Two hundred thousand cells were infected with C. psittaci and allowed to incubate at 37 degrees. Before inoculation the cells were incubated at 4 degrees for 2 hours. Once inoculated the cells were shifted to 37 degrees to synchronize the infection. At the time of harvest, the infected cells were spun down to a pellet and lysed via bead sonication. The reticulate bodies were isolated by gradient purification as outlined in Chapter 2 .

\section{Immunoprecipitation of EUO from Infected Cells}

C. psittaci infected cells were spun down at 1,000 rpm for 5 minutes at 4 degrees Celsius. Resuspended the pellet with $5 \mathrm{mLs}$ of cold phosphate buffered saline (PBS). Sonicated the solution with a probe-tip sonicator with 30 second bursts and 30 seconds on rest on ice. Repeated this step 10 times. Spun down this solution at 1,000 rpm for 5 minutes. Cross-linked the supernatant with formaldehyde (1\% final concentration) for 20 minutes. Incubated the material with glycine $(0.125 \mathrm{M}$ final concentration) to stop the cross-linking. Spun down material to a pellet at 10,000 rpm for 10 minutes. Immunoprecipitated EUO from cross-linked, infected cell pellets with the protocol outlined in Chapter 3.

\section{Southern Blot Analysis}

Genomic DNA was isolated from gradient-purified elementary bodies using the MasterPure DNA purification kit (Epicentre). The DNA was digested with BamHI, EcoRI, or XbaI restriction enzymes and the digests were electrophoresed in separate lanes on a $1 \%$ agarose gel. Radioactive probes were generated by end-labeling EUO coimmunoprecipitated DNA with ${ }^{32} \mathrm{P}$. Transfer of digested DNA and hybridization of the probes to DNA was performed as described by Southern (1975).

\section{ChIP-Cloning}

A total of two hundred thousand L929 cells were infected with C. psittaci. At 16 hours post-infection, reticulate bodies were isolated via gradient purification. The reticulate bodies were formaldehyde cross-linked. EUO was immunoprecipitated and DNA was isolated/amplified following the protocol in Chapter 3. The DNA was ligated into the pCR2.1 vector and was transformed into TOP10 cells using the TOPO TA cloning kit (Invitrogen). The clones were then sequenced. 


\section{$\left[{ }^{35}\right.$ S]-Labeling of Chlamydial Extracts}

L929 cells $\left(10^{6}\right)$ were infected with $C$. psittaci. After 15 hours post-infection, the infected cells were spun down at 1,000 rpm for 5 minutes. The pellet was resuspended in PBS containing $100 \mu \mathrm{g}$ of cycloheximide per $\mathrm{mL}$. This suspension was then incubated for 1 hour with $50 \mu \mathrm{Ci}$ of $\left[{ }^{35} \mathrm{~S}\right]$ methionine at 37 degrees Celsius. This material was spun down at 10,000 rpm for 20 minutes. The resulting pellet was resuspended in PBS and subjected to Renocal gradient purification of the reticulate bodies.

\section{Glutathione-S Tagged EUO Pulldown}

Recombinant GST-EUO was grown in E. coli and isolated using the GST purification kit (Sigma). Once isolated purified GST-EUO was prebound to glutathione beads and incubated ${ }^{35} \mathrm{~S}$-labeled chlamydial extracts. The beads were washed three times and the GST-EUO was eluted from the glutathione beads. The eluate was electrophoresed on a $13 \%$ polyacrylamide gel. The gel was dried and interacting proteins were visualized via autoradiography.

\section{RESULTS}

\section{Identification of EUO from Chlamydia Psittaci Extracts}

The first step in ChIP-cloning was the immunoprecipitation of the EUO protein from extracts. L cells were infected with $C$. psittaci and reticulate bodies (RBs) were harvested at different times post-infection. Samples from 10, 16, and 24 hours postinfection (hpi) were isolated and were subjected to western blot analysis. These time points represent late-early, mid-cycle, and mid-late stages of the infection. Due to the difficulty in obtaining sufficient gradient-purified material at immediately early time points in the cycle ( 0 to $4 \mathrm{hpi}$ ) we were restricted to using $10 \mathrm{hpi}$ as the early time point. A single band was observed to migrate above the $25 \mathrm{kDa}$ marker, which is the expected molecular size of EUO, in each sample at each time point (Figure 4-1). This band was absent when uninfected cells were probed (data not shown). EUO expression was observed to peak at the 16-hour time point.

\section{Immunoprecipitation of EUO from Chlamydia Psittaci Extracts}

I chose the 16-hour time point to immunoprecipitate EUO because of its relative abundance at this time point. To ensure that the EUO/DNA interaction was not disrupted during the immunoprecipitation of EUO, isolated RBs were formaldehyde cross-linked. RBs were purified via Renocal gradient purification and were treated with formaldehyde to crosslink DNA-binding proteins to DNA. Anti-EUO antibodies were used to immunoprecipitate EUO from this cross-linked material, which was subjected to western 


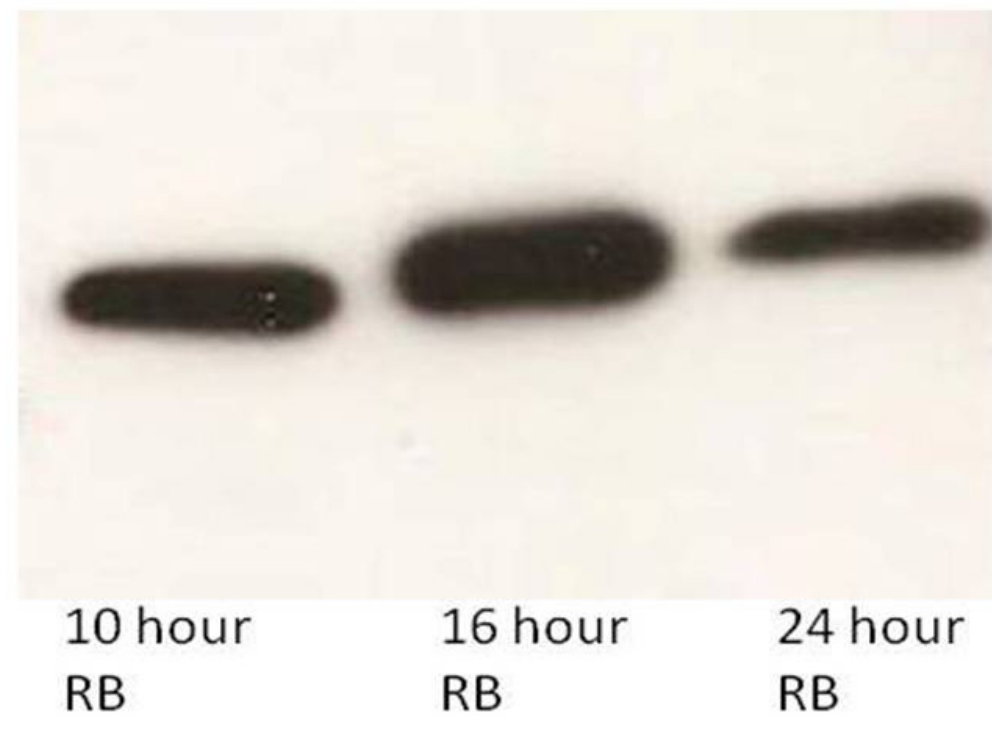

Figure 4-1. Detection of EUO in Chlamydia psittaci extracts.

RBs from 10, 16, and 24 hpi were harvested and subjected to western blot analysis. The blot was probed with antibodies to EUO. 
blot analysis. A band corresponding with the predicted molecular size of EUO was detected; this band was not present in the sample where prebleed serum was used (Figure 4-2). An interesting observation was the presence of a band around the $50 \mathrm{kDa}$ marker in the RB extract before immunoprecipitation. It is possible that through the formaldehyde cross-linking of the RBs that EUO could have been cross-linked to another protein of similar molecular size, but more likely represents a dimer of EUO. The next step in the ChIP-cloning procedure was the isolation of DNA cross-linked to the immunoprecipitated DNA. After heating at $65^{\circ} \mathrm{C}$ to break the cross-links, DNA was isolated and run on a $1 \%$ agarose gel, but no DNA was visualized (data not shown). Because the amount of DNA may have been insufficient to visualize, the Whole Genome Amplification kit was used to amplify the DNA and it was then visualized on the gel (Figure 4-3). I failed to detect DNA in the control sample (extract and prebleed) after amplification; therefore, the presence of DNA was dependent on the immunoprecipitation of the EUO protein. Once isolated, the DNA was radioactively labeled and was used to probe BamHI, EcoRI, and XbaI digested genomic chlamydial DNA from gradient purified EBs on a Southern blot (Figure 4-4). As a control, DNA from uninfected host cells was isolated and digested with BamHI. The results indicated that the immunoprecipitated DNA was of chlamydial origin and not significantly contaminated with host DNA.

\section{ChIP-Cloning Identifies Several In Vivo Targets of EUO}

Due to the lack of a microarray containing sequences from the C. psittaci genome, it was necessary to clone and sequence the EUO co-immunoprecipitated DNA for identification. The amplified, immunoprecipitated DNA was ligated into the pCR2.1 vector and transformed into TOP10 cells. These cells were grown and cells containing insert was selected via blue-white screening. To verify these clones contained insert sequence, these clones were lysed and the insert sequences were PCR amplified. Once amplified, the PCR products were analyzed on a $1 \%$ agarose gel (data not shown). Insert sequences ranged between 100-700 base pairs in size. The inserts were ethanol precipitated and sequenced. The presence of flanking linker sequences that were attached to the DNA during the amplification step caused a significant decrease in the sequencing efficiency. Nonetheless, thirty clones were sequenced. Once sequenced, the coimmunoprecipitated DNA was used in BLAST searches to identify where in the genome EUO was binding and a list of the genes identified was compiled (Table 4-1). Five genes were membrane proteins, four genes encoded for putative export proteins, and eight genes encoded for hypothetical proteins of unknown function, indicating that EUO binding was not specific for a particular family of genes. Also, the identified genes were scattered throughout the genome, indicating that EUO binding was not restricted to a particular region. 


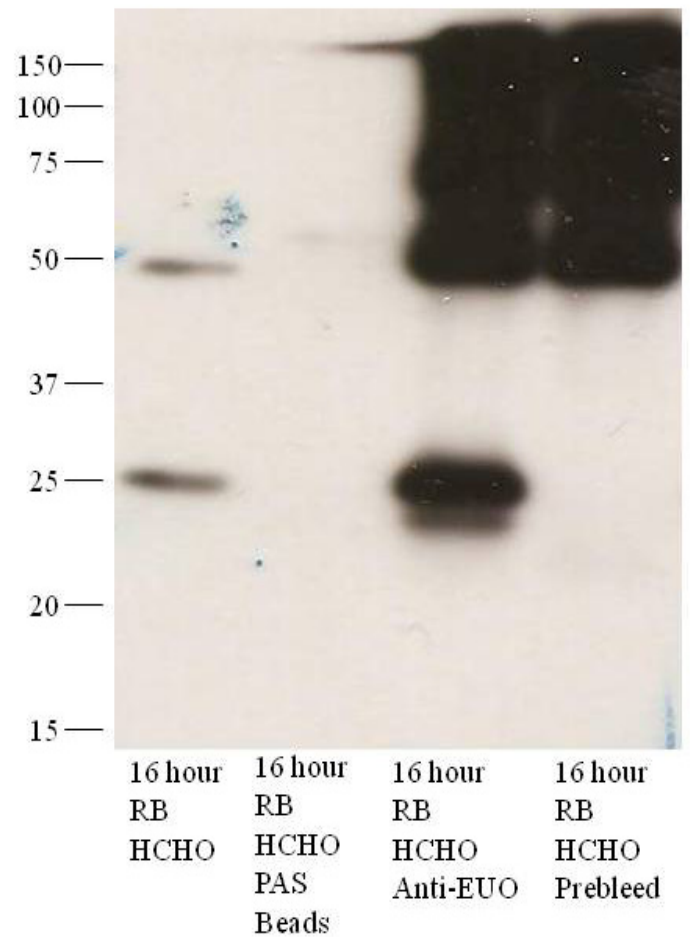

Figure 4-2. Immunoprecipitation of EUO from formaldehyde cross-linked Chlamydia psittaci RBs.

Sixteen hpi RBs were cross-linked and EUO was immunoprecipitated. Extracts were immunoprecipitated with prebleed serum as a control. Formaldehyde is denoted as $\mathrm{HCHO}$. 


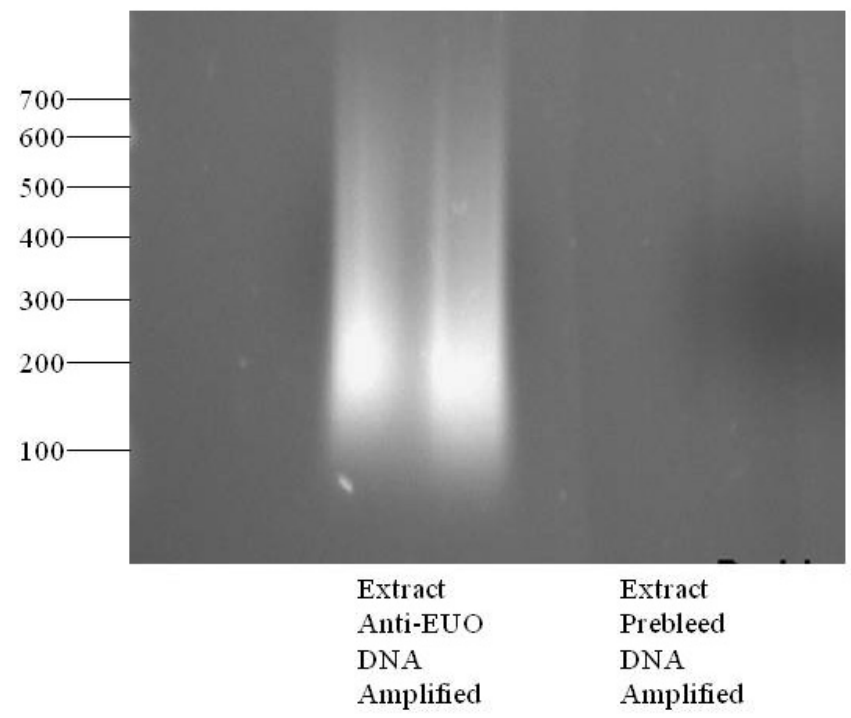

Figure 4-3. Amplification of EUO co-immunoprecipitated DNA.

DNA was isolated from EUO immunoprecipitations. Once isolated, DNA was amplified and run on a $1 \%$ agarose gel. DNA isolated from prebleed immunoprecipitations was amplified and used as a control. Base pair markers are indicated. 


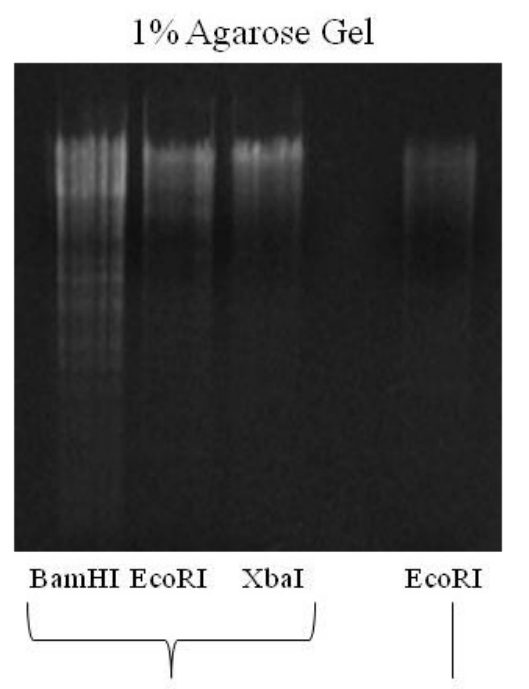

C.psittaci DNA

Host cell DNA

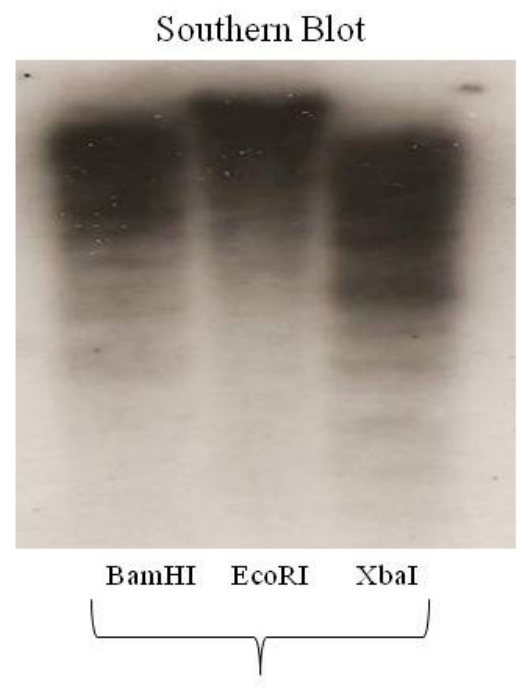

C.psittaci DNA

Figure 4-4. Southern blot analysis of EUO co-immunoprecipitated DNA.

Genomic DNA was isolated from EBs, digested with the restriction enzymes BamHI, EcoRI, or XbaI, and run on a $1 \%$ agarose gel to prepared a Southern blot. EUO coimmunoprecipitated DNA from $16 \mathrm{hpi}$ RBs was radioactively labeled and allowed to hybridize to the blot. As a control, host cell DNA was digested with EcoRI and ran in the far right lane. No hybridization was seen when host cell DNA was probed with the labeled DNA (not shown). 
Table 4-1. List of genes identified with BLAST search of EUO coimmunoprecipitated DNA.

\begin{tabular}{ll}
\hline Clone Number & \multicolumn{1}{c}{ Gene Name } \\
\hline 1 & Putative inner membrane protein \\
2 & Putative general secretion protein \\
3 & Putative exported protein \\
4 & Conserved hypothetical protein \\
5 & Conserved hypothetical protein \\
6 & Putative ClpB ATPase stress related protein \\
7 & Putative type III export protein \\
8 & DNA mismatch protein (MutL) \\
9 & 30s ribosomal protein s7 \\
10 & Putative transport-related membrane protein \\
11 & Putative regulatory protein (V1) \\
12 & Putative serine/threonine protein kinase \\
13 & Putative inner membrane protein \\
14 & Conserved hypothetical serine-rich protein \\
15 & Putative exported protein \\
16 & Putative thiol:disulfide exchange protein \\
17 & Putative hydrolase \\
18 & Conserved hypothetical protein \\
19 & Conserved hypothetical protein \\
20 & Conserved hypothetical protein \\
21 & Uncharacterized P-loop hydrolase \\
22 & YjgP-YjgQ family protein \\
23 & Conserved hypothetical protein \\
24 & Acetyl-CoA carboxylase \\
25 & YjgP-YjgQ family protein \\
26 & Putative hydroxyethylthiazole kinase \\
27 & Putative inner membrane protein \\
28 & Conserved membrane protein \\
29 & Putative division protein A (gidA) \\
30 & Conserved hypothetical protein \\
\hline &
\end{tabular}




\section{Immunoprecipitation of EUO from Infected Cells}

In the studies just described, it is possible that the EUO/DNA interactions could have been disrupted during RB purification, before the cross-linking of the RBs. Another concern is the time point in which the EUO/DNA complexes were isolated. EUO was isolated at $16 \mathrm{hpi}$, the time where EUO expression peaks in order to obtain sufficient material for analysis. Because EUO is expressed very early it may bind more specifically at immediately early times or at least bind to different sequences of DNA than at $16 \mathrm{hpi}$. In an attempt to address these concerns, EUO immunoprecipitations from formaldehydetreated extracts of cells at early time points in the developmental cycle the cycle were examined. (Attempts at immunoprecipitating EUO from intact cross-linked infected host cells were unsuccessful; not shown.) We attempted to immunoprecipitate EUO at 2 hpi. EUO was not detected in the immunoprecipitation from extracts prepared at 2 hpi (Figure 4-5) but was detected in infected cell extract prepared at 3 and 4h hpi (Figure 4-6 and Figure 4-7). EUO was not detected when the material was immunoprecipitated with prebleed serum, indicating that the immunoprecipitation of EUO from the cross-linked infected cells was dependent on the presence of EUO serum.

\section{Isolation and Sequencing of EUO Co-Immunoprecipitated DNA from Infected Cells}

DNA was isolated from 3 and 4 hpi immunoprecipiates, but was detected on agarose gels only in the 4-hours sample (Figure 4-8). This DNA was cloned and sequenced, but sequencing revealed only DNA of host origin. The presence of DNA was dependent on the immunoprecipitation of EUO; therefore, it would appear that EUO during the lysis of the infected cells was being released from reticulate bodies where it would bind host DNA. The ability of EUO to bind DNA other than that from chlamydia was previously demonstrated in the first study identifying DNA (Wichlan and Hatch 1993) and the results here further confirm that EUO binding is relatively non-specific.

\section{DNase Contains Proteolytic Activity}

An attempt was made to eliminate host DNA in infected cell extracts by treating the extracts with DNase before the addition of formaldehyde, but the addition of DNase resulted in the inability to immunoprecipitate EUO (data not shown). To confirm that DNase treatment was responsible for the inability to immunoprecipitate EUO, an experiment was performed in which a 4 hpi extract was divided in two equal parts, only one of which was treated with DNase. Both samples were formaldehyde cross-linked and EUO was subjected to immunoprecipitation. EUO was only detected in the sample that was not treated with DNase (Figure 4-9). The effect of DNase treatment on the capacity to detect EUO in 4-hour infected cells extracts directly (without immunoprecipitation) was investigated (Figure 4-10). EUO was not detected in the sample that was DNasetreated, suggesting the possibility that the DNase was contaminated with proteolytic enzymes. One interesting observation was the presence of bands at the $50 \mathrm{kDa}, 75 \mathrm{kDa}$, 


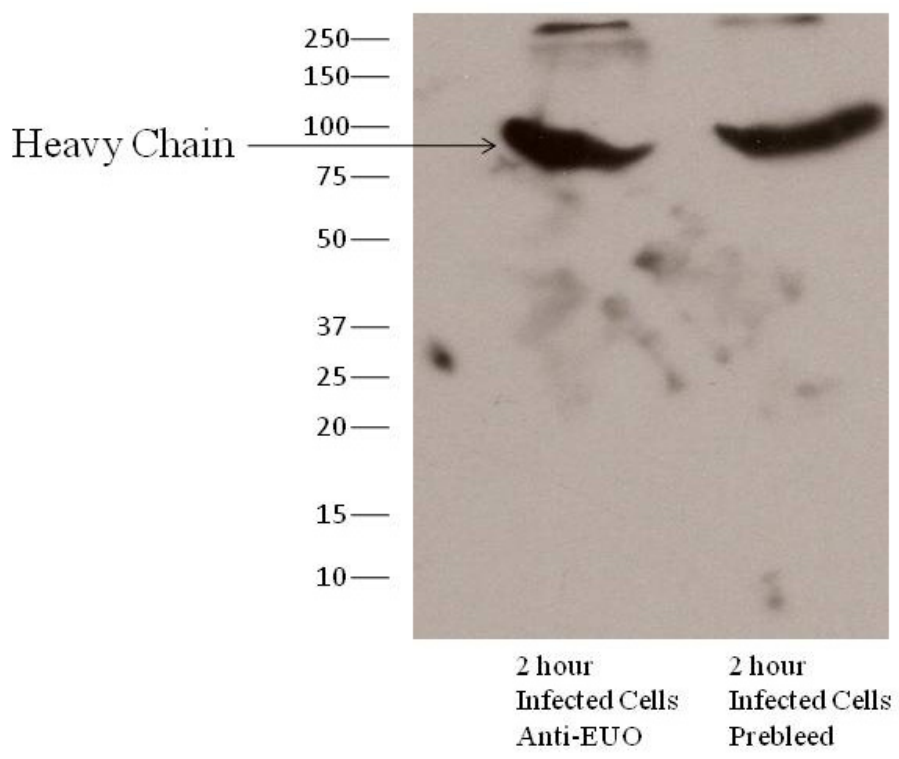

Figure 4-5. Immunoprecipitation of EUO from cross-linked infected cells at 2 hpi.

Cells were infected with $C$. psittaci and at two hpi the cells were collected and subjected to EUO immunoprecipitation. Immunoprecipitation with prebleed serum was used as a negative control. 


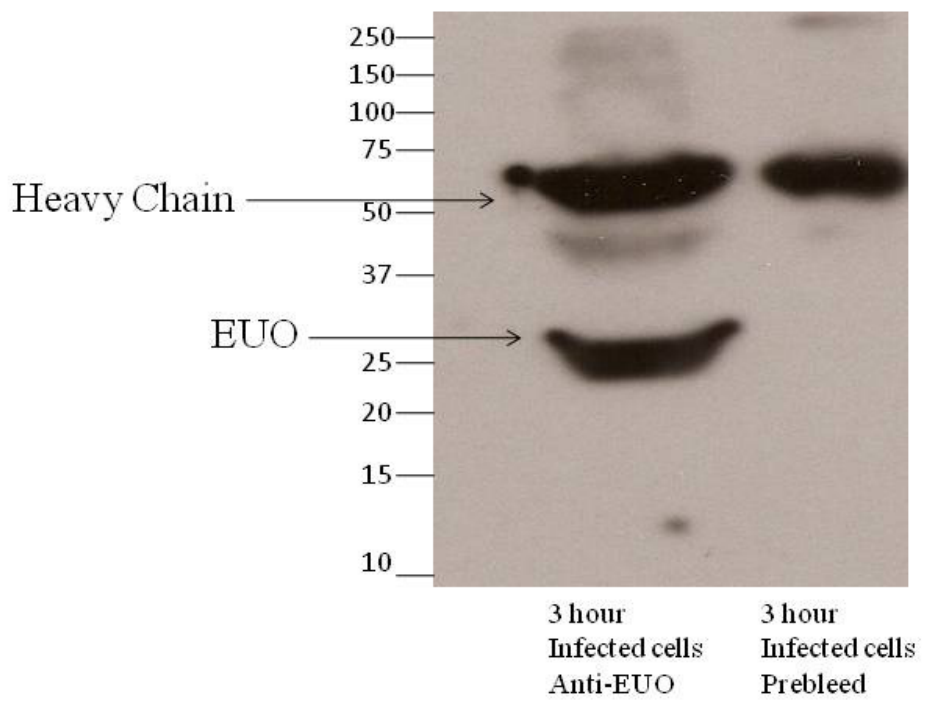

Figure 4-6. Immunoprecipitation of EUO from cross-linked infected cells at 3 hpi.

Cells were infected with $C$. psittaci and at three hpi the cells were collected and subjected to EUO immunoprecipitation. Immunoprecipitation with prebleed serum was used as a negative control. 


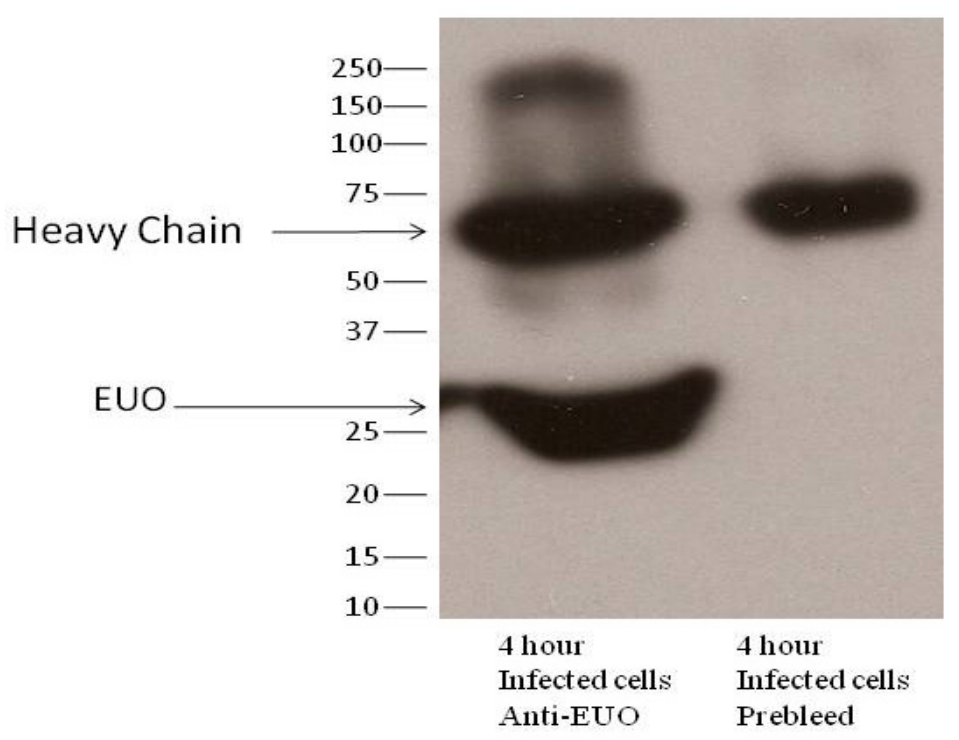

Figure 4-7. Immunoprecipitation of EUO from cross-linked infected cells at $4 \mathrm{hpi}$.

Cells were infected with C. psittaci and at four hpi the cells were collected and subjected to EUO immunoprecipitation. Prebleed immunoprecipitation was used as a negative control. 


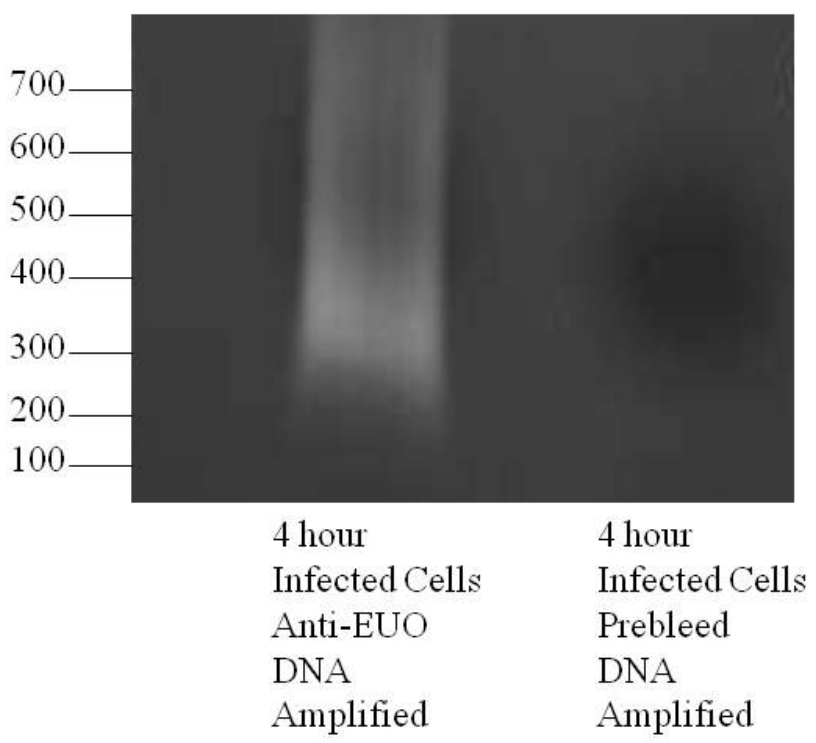

Figure 4-8. Amplification of EUO co-immunoprecipitated DNA.

EUO was immunoprecipitated from formaldehyde cross-linked cells at 4 hpi. The coimmunoprecipitating DNA was isolated and amplified (Lane 1). DNA isolated from prebleed immunoprecipitations (Lane 2) of the infected cells was used as a negative control. Markers denote size in base pairs. 


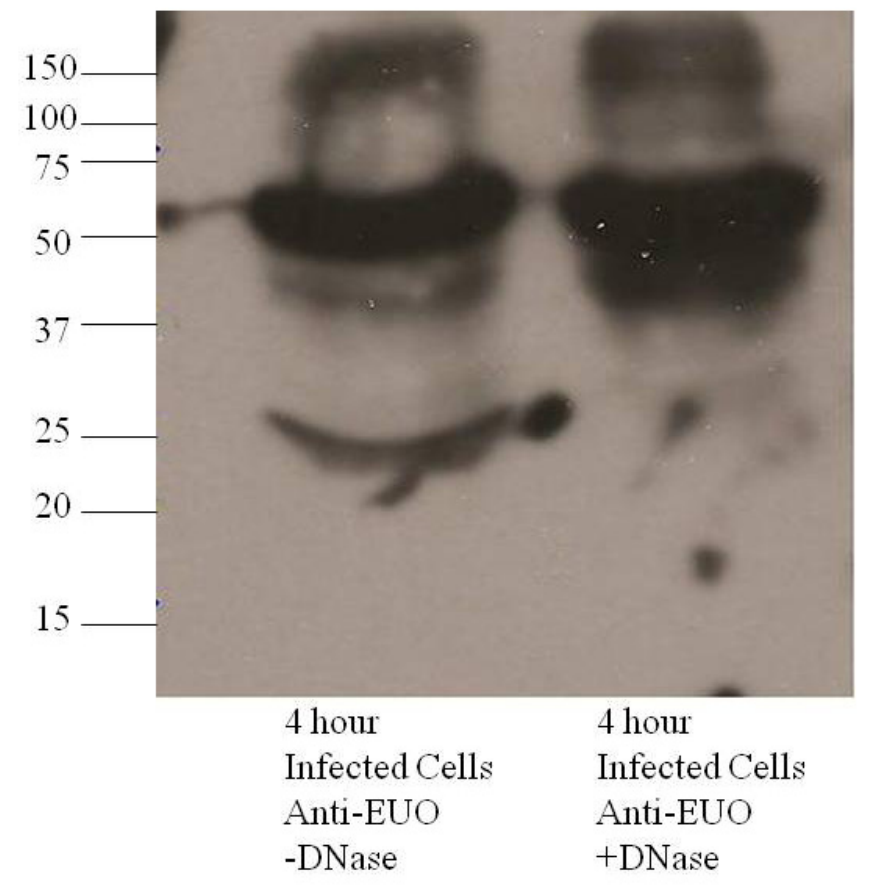

Figure 4-9. Effect of DNase treatment on EUO immunoprecipitation.

Four-hour infected cells were lysed and extracts were formaldehyde cross-linked and divided into two equal parts and incubated with or without DNase. EUO was immunoprecipitated and western blot analysis was performed to detect EUO. Lane 1, DNase; Lane 2, +DNase. 


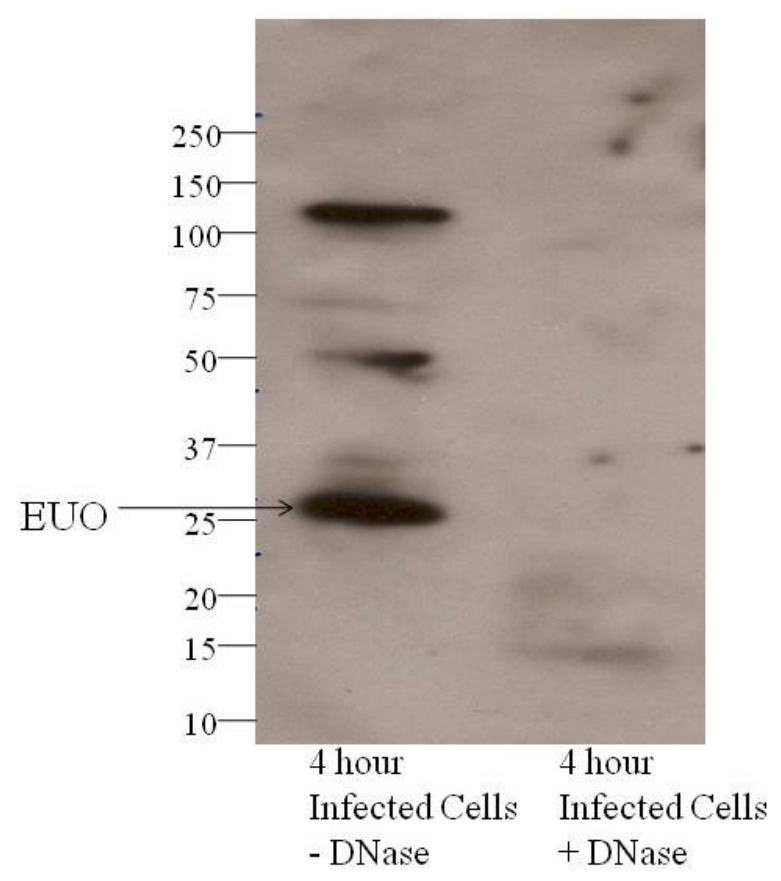

Figure 4-10. Effect of DNase treatment of infected cell extracts on EUO.

Four-hour infected cells were isolated and divided in two equal samples. One sample did not receive DNase treatment (Lane 1) and the other sample was treated with DNase (Lane 2 ). Both samples were treated with formaldehyde before electrophoresis. The samples were subjected to western blot analysis and were probed with EUO antibodies. 
and $100 \mathrm{kDa}$ in the sample that was not treated with DNase. Because the samples were cross-linked, these bands likely represent multimers of EUO or other proteins crosslinked to EUO. Next, I examined if the effect seen with DNase treatment was specific for EUO, a minor protein, or if other proteins were affected. Sixteen-hour purified RBs were treated with or without DNase. Coomassie-stained blots were used to identify differences in banding patterns, and western blots of the samples were also probed with antibodies to heat shock protein 60 (HSP-60). Treatment with DNase resulted in the disappearance of multiple bands on Coomassie blot including the 37-40 kDa MOMP band (Figure 4-11A) and the HSP-60 protein was partially degraded in the DNase-treated sample (Figure 4-11B). Similar results were obtained with DNase obtained from other labs, including molecular biology grade DNase. It was concluded that DNase could not be used to eliminate host DNA because of contaminating proteolytic activity. Consequently, further experimentation on EUO-bound DNA at very early time points were not attempted. DNase treatment of lysates of infected cells is routinely performed prior to purification of EBs and RBs on Renocal gradients. It is curious that deleterious effects of the proteolytic activity have never been noticed.

\section{Binding of Proteins to EUO Was Not Detected via Co-Immunoprecipitations and GST-EUO Pulldowns}

It has been established that EUO is capable of binding DNA but the possibility of EUO binding other proteins needed to be investigated. The presence of the two bands seen with the detection of cross-linked infected cells in Figure 4-10 lends credence to the possibility that EUO may bind other proteins. To address this possibility, GST-EUO pulldowns of extracts of RBs that were labeled with $\left[{ }^{35} \mathrm{~S}\right]$ methionine/cysteine and EUO immunoprecipitations from the radiolabeled extracts were performed. Although several bands were observed in both GST-EUO pulldown (Figure 4-12) and the immunoprecipitation (Figure 4-13), these same bands were also noted in with about the same intensity in control samples (beads without GST-EUO and immunoprecipitation with pre-bleed serum). I conclude that EUO does not bind other proteins in 16-hpi RBs that can be detected within the limits of these experiments. It is particularly noteworthy that radiolabeled EUO was detected in the immunoprecipitate. If an RB protein were bound to EUO in a one-to-one ratio, it should have been detected.

\section{DISCUSSION}

Previous studies (Wichlan and Hatch 1993; Zhang, Howe et al. 2000) and those reported in Chapter 3 all demonstrated that recombinant EUO (rEUO) does not bind to DNA randomly but that binding in vitro is relatively non-specific. The study reported in this chapter was undertaken to identify strong and weak sites to which native EUO binds to DNA in vivo, with the hope that a more specific pattern of binding could be identified. The study suffered from several limitations. Most importantly, analysis is ideally carried out by formaldehyde-fixation of whole cells under specific conditions (for example, in the presence of factor $\mathrm{X}$ or at time period $\mathrm{Y}$ ). In the case of chlamydiae, intact infected 

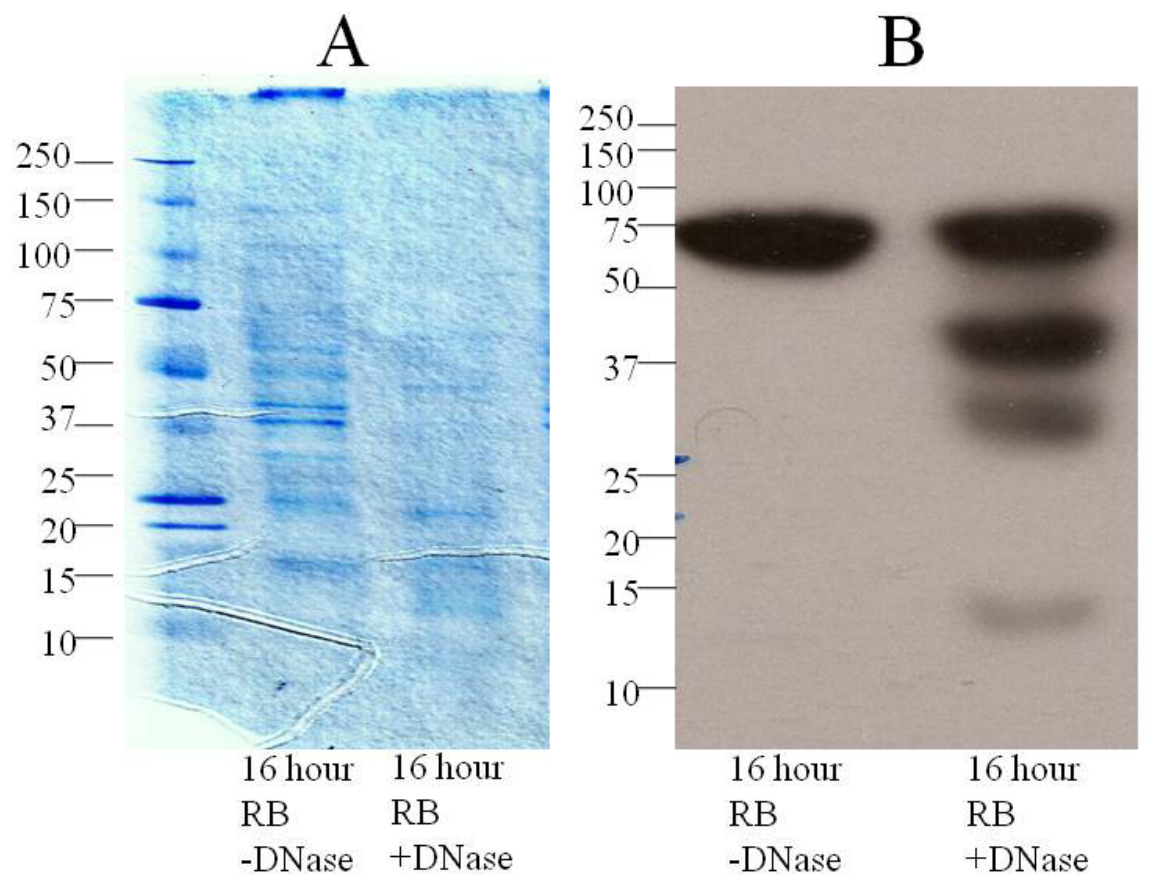

Figure 4-11. Effect of DNase on chlamydial extracts and HSP-60 detection.

Sixteen-hour, gradient-purified RBs were isolated and were incubated in the absence (Lane 1) or the presence of DNase (Lane 2). The samples were separated on 13\% polyacrylamide gels and Coomassie stained (Panel A) or subjected to western blot analysis to detect HSP60 (Panel B). 


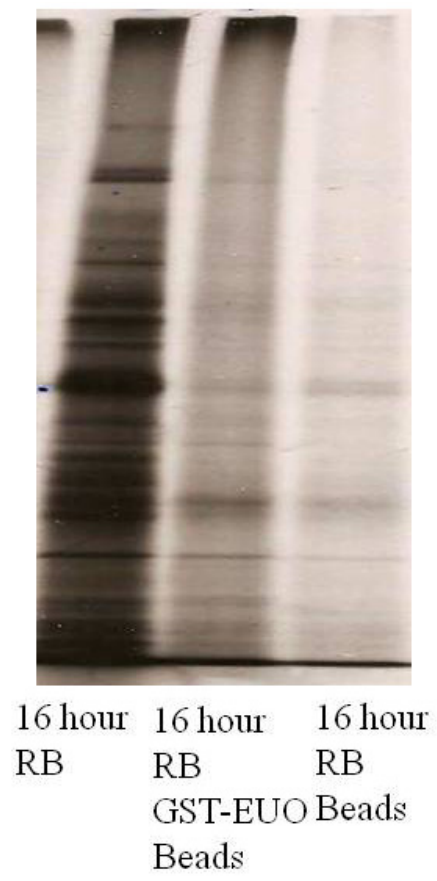

Figure 4-12. GST-EUO pulldown from extracts of $\left[{ }^{35} \mathrm{~S}\right]$-labelled RBs.

Radioactively labeled 16-hpi RBs were lysed, GST-EUO was pulled down from the lysate, and the material in the pulldown was analyzed by SDS-PAGE autoradiopraphy. Lane 1, 16-h RB extract; lane 2, Pulldown by GST-EUO-glutathione beads; lane 3, Pulldown by glutathione beads in the absence of GST-EUO. 


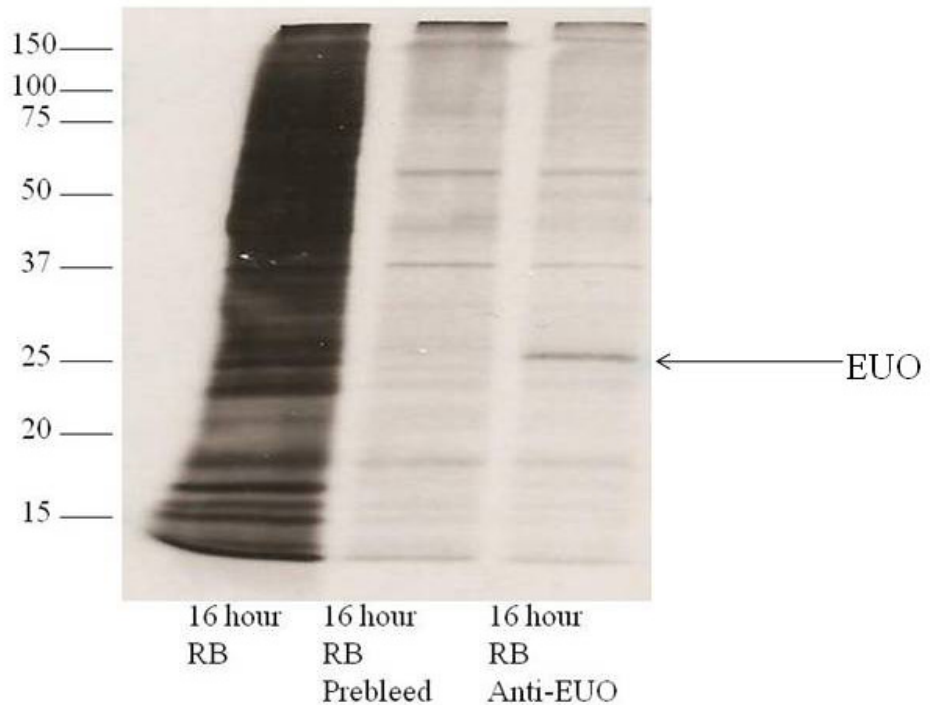

Figure 4-13. EUO immunoprecipitation from extracts of $\left[{ }^{35} \mathrm{~S}\right]$-labelled RBs.

Radioactively labeled 16-hpi RBs were lysed, EUO was immunoprecipitated, and the material was analyzed by SDS-PAGE autoradiopraphy. Lane 1, extract alone; lane 2, precipitate using prebleed serum; lane 3, precipitate using anti-EUO serum. 
cells would be fixed at early, middle, and late time points. Unfortunately, I was not able to immunoprecipitate EUO from whole fixed infected cells. Thus, I settled on analysis of fixed purified RBs. Previous studies in the Hatch lab (Crenshaw, Fahr et al. 1990) demonstrated that host-free RBs transcribe DNA in a stage-specific manner, suggesting that analysis of purified RBs should yield results comparable with intracellular events. However, it was not possible to isolate sufficient purified RBs that yielded EUOprecipitable DNA earlier than 16 hpi. Attempts to analyze RBs in fixed crude extracts at early times post infection yielded only host DNA bound to EUO, and attempts to eliminate host DNA from the extracts by treatment with DNase apparently eliminated EUO by proteolysis.

There is no clear explanation for this phenomenon other than the contaminating proteolytic activity must be able enter RBs, perhaps damaged during lysis of the host cells. In any event, data was obtained from 16 hpi that likely reflect events that took place before harvesting and purification, although this cannot be proven. The 16-hpi RB analysis yielded results that were similar to previous, strictly in vitro studies: binding was not specific for a particular region of the genome, binding was to ORFs rather than upstream regulatory regions (this may be a function chance; about $75 \%$ of the genome encodes ORFs), there was no stage-specific pattern to the bound ORFs, and no consensus sequence was identified within the binding fragments. Taken together with the findings of previous studies, including those reported in Chapter 3, I conclude that the working hypothesis that EUO is a regulator of stage-specific gene regulation is incorrect. What then is the function of EUO? Here is a summary of what is known about EUO. 1) It is encoded by the genomes all Chlamydia species and a highly homologous gene is found in proto-chlamydia that diverged from the genus Chlamydia about a billion years ago. 2) EUO protein is absent in EBs but the euo gene is one of the first and most actively expressed genes within 2 hours of infection. 3) EUO continues to be made during the logarithmic phase of growth, but disappears late in the cycle. 4) EUO is a minor protein which is never present in sufficient amounts to be clearly identified by Coomassie-stained gels. 5) EUO binds to DNA in vitro. 6) Binding is not confined to chlamydial DNA. 7) AT-rich sequences are preferred (not confirmed by my studies). 8) Binding to chlamydial DNA in vitro is not random but is relatively non-specific. 9) Binding of EUO to DNA in vitro is sufficiently tight to produce DNase footprints (Zhang, Douglas et al. 1998; Zhang, Howe et al. 2000). 10) EUO binds to ORFs and intergenic regions. From these observations, I propose the following hypothesis: EUO is not a stage-specific regulator of transcription per se but rather is a general DNA binding protein that facilitates transcription, somehow boosting transcription at the very beginning of the cycle through the logarithmic phase. The limited number of EUO molecules per RB suggests that EUO does not facilitate transcription of all genes, but the relatively nonspecific binding suggests many genes are affected. EUO is degraded sometime during the late phase, thus whereas it can bind to late gene sequences, it is not available to facilitate transcription of late genes. My studies do not suggest a mechanism by which EUO may facilitate transcription, but the failure to detect binding specifically near promoters suggests that EUO does not function during initiation events. Alternative functions for EUO will be discussed in the General Discussion chapter. It is hoped that this loose model will serve to direct future studies on the function of EUO. 


\section{CHAPTER 5. GENERAL DISCUSSION}

Our current understanding of mechanisms by which stage-specific gene expression is regulated is reviewed in Chapter 1, but specifics remain largely unknown. My studies investigated chlamydial gene regulation by attempting to answer two questions: Do sigma protein levels throughout the cycle suggest regulation by the sequential protein expression of stage-specific sigma factors in a cascade fashion and what are the DNA binding sites to which EUO protein bind? The transcription profiles of the three chlamydial sigma genes during the cycle have been reported, but the relative level of sigma proteins has not. Plus, at least one well-known gene, $h c t B$, is transcribed by alternative sigma factor 28 . The euo gene is one of the first and most actively expressed genes early in the cycle but is not transcribed late in the cycle and EUO protein is not present in EBs. Previous studies found that EUO binds to DNA in vitro, but that binding, while not random, is relatively non-specific. Therefore, I took a more global approach than previous studies to identify EUO binding sites in vitro and in vivo with the rationale that identification of sites might reveal the function of EUO.

\section{SIGMA FACTORS ROLE IN TRANSCRIPTIONAL REGULATION IN CHLAMYDIA}

\section{Expression of the Sigma Factor Proteins}

IFA and western blot studies were undertaken to examine the working hypothesis that analysis of sigma protein levels during the cycle may identify one or more sigma factors as a potential regulator of stage-specific gene expression. The points in the cycle tested were 10 to $48 \mathrm{hpi}$. Sigma 66, the major sigma was present at all times as expected. Also, its present late in the cycle supports the in vitro transcription studies of Fahr et al. (1995) that sigma 66 initiates transcription from several known late-stage promoters. Possibly because of the insolubility of recombinant protein, not one chlamydial gene has been confirmed by in vitro transcription studies to be regulated by sigma 54 . However, two potential sigma 54 promoters have been identified on the basis of predicted sequence similar to the bacterial sigma 54 consensus, and it has been suggested that sigma 54 is a late-phase sigma factor (Mathews and Timms 2000). Whereas I found RB cytoplasmic sigma 54 protein levels, as detected by monoclonal antibodies, to be present late in the cycle, the protein is prominently present as early as $10 \mathrm{hpi}$ and appears to be RB-specific at late times. Therefore, I concluded that sigma 54 does not appear to be a stage-specific sigma factor, although regulation of unidentified late genes that are expressed in the late logarithmic phase cannot be excluded at this time. In contrast, the pattern of expression of sigma 28 does suggest that it may play a role in early EB development. Although protein can be detected as early as $10 \mathrm{hpi}$, levels peaked at about 16-24 hpi, at the time when late gene transcription is first detected, including the known sigma 28-transcribed gene, $h c t B$. In summary, my findings support a partial cascade pattern of chlamydial gene expression in that sigma 28 is best expressed when early late genes are transcribed. 
Future studies need to investigate aspects of sigma proteins that I did not address. Perhaps most notably, very early times points were not examined. This could easily be accomplished by IFA studies. Preliminary studies not reported here do indicate that sigma 28 protein is not detected in 4 hpi RBs. Sigma 54, present in EBs (Figure 2-10) is present in infecting EBs, as detected by polyclonal serum and thus also found at 4 hpi (Sigma 54 was not detected in EBs by the monoclonal serum). However, IFA staining at 10 hpi (Figure 2-9) suggests the protein in EBs had undergone degradation and may not be functional. Another aspect that requires more study is the time of expression of alternative sigma factor regulators. Our lab does not have access to antibodies against the predicted known sigma 54 regulator, $\mathrm{CtcB}$, the equivalent or $\mathrm{NtrC} \mathrm{(Koo} \mathrm{and} \mathrm{Stephens}$ 2003); thus, CtcB was not investigated. As discussed in Chapter 1, in some bacteria, the activity sigma 28-like factors is regulated by a complex system of anti- and anti-antisigma factors, some of which (RsbW, RsbU, and RsbV1 and V2) are encoded by chlamydial genomes. Our lab has generated polyclonal antibodies to recombinant forms of these factors. In studies not reported here, I found that $\mathrm{W}$ and $\mathrm{U}$ factors were difficult to detect by IFA and western blot (indecisive results were obtained). Both V proteins were easily detected by both methods, but were present at all times post-infection and in EBs, a finding not offering much insight into how they might regulate sigma 54 in a stage-specific manner. Moreover, two studies (Hua, Hefty et al. 2006; Karlinsey and Hughes 2006) suggest that the anti-sigma factor RsbW does not bind to chlamydial sigma $28(\mathrm{~V}, \mathrm{~W}$, and $\mathrm{U}$ do interact as expected), casting doubt on the role of Rsb proteins in the regulation of sigma 28 in chlamydiae (see Chapter 1).

The scope of my study was to monitor the protein levels of the three chlamydial sigma factors during the cycle. Clearly, the most important future study would be to monitor sigma factor activity during the cycle. At present, the only approach to do this in chlamydiae would be to further develop in vitro transcription assays using extracts of RBs. A second best approach would be to identify predicted promoters in silico, determine the time of expression of their cognate genes, and use recombinant sigmas factors in an in vitro transcription system to confirm predictions. This approach is tedious but has been accomplished for several late genes (Yu and Tan 2003); however, recombinant sigma 54 activity has not been reported, perhaps because of its insolubility. A non-in silico approach to identify the promoters of each sigma factor would be to perform a ChIP-to-Chip analysis using recombinant sigma factors in a manner similar to what was done with recombinant EUO in this study (Chapter 3). A limitation of this approach is that, with the exception of sigma 28-like factors, sigma factors tend to bind DNA non-specifically in the absence of the other RNA polymerase subunits.

\section{Detection of an Unknown Antigen with Sigma 54 Polyclonal Serum}

An interesting observation of the sigma factor study was the detection of an antigen in the chlamydial outer membrane complex by the polyclonal sigma 54 serum. Assuming that the IFA results reflect reaction with some form of sigma 54 antigen, it is possible that the polyclonal serum recognizes a membrane-bound form of sigma 54 not recognized by the monoclonal antibody that only detects a cytoplasmic form of sigma 54 . 
If this speculation is correct, sigma 54 may be in the class of extracytoplasmic function (ECF) sigma factors. As reviewed by Helmann (2002), ECF sigma factors contain a transmembrane protein that functions as an anti-sigma factor that binds and inhibits the cognate sigma factor. Upon receiving a stimulus from the environment, the sigma factor is released and can bind to RNA polymerase to stimulate transcription of its respective genes. Similar to ECF sigma factors, sigma 54, in its inactive form, may be bound to the chlamydial membrane and when activated detaches from the membrane and translocates to the cytoplasm where it is able to bind RNA polymerase, thus allowing the expression of sigma 54 regulated genes.

Another explanation behind sigma 54 antiserum staining the chlamydial membrane is that the polyclonal serum cross-reacted with an unknown antigen located in the outer membrane of chlamydiae. Western blot analysis using sigma 54 polyclonal serum revealed bands being detected at the top of the gel in the loading wells, possibly indicating a supra-molecular complex that is not capable of migrating into the gel (not shown). Unfortunately, attempts to lower the polyacrylamide percentage of the gel to allow this complex to enter into the gel were unsuccessful. If the sigma 54 polyclonal serum does cross-react with an antigen structurally unrelated to sigma54, how was this cross-reactive activity generated by using recombinant sigma 54 protein as the antigen? One possibility is that the rabbit raised antibodies to the adjuvant in the inocculum. Adjuvants contain complex wall substances from mycobacteria and Brown and Rockey (2000) speculated that an antibody was thusly generated in their lab that cross-reacts with peptidoglycan in the septum in dividing RBs. It would seem unlikely that our polyclonal reacts with peptidoglycan in that peptidoglycan has not been identified in stoichometric amounts in EBs. However, reaction with some other supra-molecular complex is possible. Also, the insoluble nature of the recombinant sigma 54 used as immunogen, rather than adjuvant, may have generated antibodies that recognize three-dimentional antigens in the OMC. The generation of this serum did, however, produce antibodies against sigma 54, based on the ability of the serum to detect recombinant sigma 54 on western blots. Thus, the possibility that some complex form of sigma 54 is indeed located in the OMC and plays a structural role cannot be dismissed.

\section{BINDING STUDIES OF THE DNA-BINDING PROTEIN EUO}

Previous studies clearly demonstrated that EUO binds to DNA in vitro in a nonrandom fashion, but with little specificity. The working hypothesis of the studies reported in Chapters 3 and 4 was that a global in vitro analysis of EUO binding and/or an in vivo analysis of DNA fragments bound by native EUO will identify strong and weak binding sites that will lead to a prediction of EUO function. The genes and fragments identified in these studies largely confirmed the earlier studies: strong binding sites were randomly distributed throughout the genome and did not fall into any stage-specific or functional category. Also, the binding sites did not allow the derivation of a consensus binding site. Although my preconceived bias was that my studies would reveal a few sites upstream from either early and/or late stage genes (a bias implied in my working 
hypothesis), my results did allow me to generate an alternative hypothesis for the function of EUO and to generate a very preliminary model for the function of EUO.

\section{Discussion of the EUO Model}

The model is preliminary and not directly supported by experimental evidence. It was derived on the basis of what is known about EUO; mainly that it binds to DNA with relatively little specificity. There is little information in the literature to provide clues as to how EUO might specifically function in this model. Previous studies suggested EUO prefers AT-rich sequence beg the possibility that EUO may bind to bends and kinks in DNA to in some way promote transcription. Also, one might expect EUO to bind preferentially to promoter regions that are AT-rich. In fact, EUO produces a broad DNase footprint in the omcAB promoter region (Zhang, Douglas et al. 1998). However, neither my studies nor those of Zhang et al. (2000) support preferential binding to promoters. These findings notwithstanding, a peculiar aspect of many chlamydial promoters is an "AT box" between the -35 and -10 promoter elements that enhances transcription in vitro (Douglas and Hatch 1995; Tan, Gaal et al. 1998). It is possible that EUO interacts in some way with AT boxes.

Future studies to test the model will be challenging. Surrogate studies in E. coli should be attempted but likely will be negatively impacted by the fact that recombinant EUO forms an inclusion body in E. coli, and even the small amounts of EUO that may be soluble in E. coli likely will bind non-specifically to DNA in a manner reminiscent of the chlamydial histone like proteins and be toxic. In fact, the Hatch recombinant clone dies during the late logarithmic phase of growth. Also, the functional interaction of EUO, a chlamydia-specific protein, with DNA may be peculiar to some aspect of chlamydial DNA or require a protein partner for function that is not in E. coli or that lacks the interactive motif. More likely to succeed but still a major "tour-de-force" undertaking would be in vitro transcription studies. Some of the problems associated with this approach include the insolubility of recombinant EUO, the lack of specific knowledge of DNA templates to be examined, the likely necessity to include either purified chlamydia RNA polymerase or functional recombinant proteins, and the possible need of as yet unidentified auxiliary proteins.

\section{EUO Protein Interaction with Other Proteins and Its Degradation}

In an attempt to identify EUO-interacting proteins that may be responsible for binding specificity, I performed immunoprecipitations from radioactively labeled extracts but results suggested that EUO did not possess a binding partner. It is plausible that the sensitivity of the assay was not sufficient to detect binding partners. If EUO does have a binding partner, it is conceivable that only a certain population of EUO molecules binds to the interacting protein at any particular time. This assumption combined with the fact that EUO is a minor protein further diminishes the probability that a binding partner will be detected. It is also possible that the addition of formaldehyde to the RBs cross-linked 
EUO and its binding partner in a manner that would not allow for this complex to be immunoprecipitated. To overcome these obstacles, two hybrid assays could be performed. Using EUO as bait, one could scan a genomic library of chlamydial proteins in an attempt to identify any interacting partners. Although this would negate the possibility that physiological conditions of the RB may play a role in EUO binding another protein, it would eliminate the dilemma of not having enough material to detect a possible binding interaction.

In my initial attempts to perform ChIP-to-Chip analysis, I cross-linked $C$. trachomatis serovar D extracts and immunoprecipitated EUO. Using polyclonal serum, I was able to detect EUO in extracts, but a discrepancy in size was noted between the size of EUO seen in extracts and the size of immunoprecipitated EUO from these extracts. It is possible the smaller species was a degradation product of the full-length protein. EUO degradation could serve as a mechanism to regulate its function, and understanding the mechanisms for this enzymatic activity should be investigated.

Although I was able to immunoprecipitate the larger species of EUO, I did not observe the smaller species being immunoprecipitated. An explanation for the ability to immunoprecipitate the full-length species and not the degraded species of EUO is that during the immunoprecipitation, serum is able to bind the native conformation of the fulllength species and not the native conformation of the degraded species. The reason for the detection of both species on the western blot is that the serum was capable of recognizing both species when denatured. Interestingly, the immunoprecipitation of the full-length species did not result in the co-immunoprecipitation of DNA. It is possible that the smaller species is the active form of EUO and is responsible for the binding of DNA. Several proteins, such as caspases, require processing before becoming active, therefore, it can be conceived that EUO needs to be processed in order to bind DNA. Further analysis, such as southwestern blots using a known EUO binding region, the CRP operon, to probe $C$. trachomatis serovar D extracts would help in evaluating if the smaller species is the only species capable of binding DNA.

It is curious that a possible degraded species of EUO was observed in extracts from $C$. trachomatis serovar D but was never been observed in C. trachomatis serovar L2 or $C$. psittaci extracts. The detection of the degraded species of EUO in the $C$.

trachomatis serovar $\mathrm{D}$ extracts could be the result of varying antibody qualities between the serums but it could also be that this phenomenon is specific to C. trachomatis serovar D. Comparing the genomes of Chlamydia spp. revealed the presence of species specific and serovar specific genes (Kalman, Mitchell et al. 1999; Shirai, Hirakawa et al. 2000; Read, Myers et al. 2003). It is believed that the differences between the genomes may be responsible for the differences observed in their growth and tissue tropism of various species of Chlamydia. The gene coding for EUO is present in each genome sequenced but factors that affect EUO's activity may be species/serovar specific. It can be speculated that the degradation of EUO may be specific for a particular species or serovar of Chlamydia and may be responsible for the differences in growth patterns or the ability to infect different tissues. 


\section{In Vitro Binding Specificity of EUO}

Primary DNA sequence may not be the sole determinant for EUO binding; rather, DNA conformation may be responsible for the affinity of EUO for particular regions of DNA. Kim et al. showed that the conformational status of DNA was an important determinant for the sequence-specific binding of the tumor suppressor protein, p53 (1997). Another study has demonstrated the ability of a protein to bind particular regions of DNA without the requirement of sequence specific motifs altogether. Gholer et al. mutated the DNA-binding domain of the tumor suppressor, p53, which disrupted its sequence specific binding characteristic but conferred it with the ability to bind specific structures of DNA (2005). Therefore, analyzing sequence alone may not be sufficient in identifying the EUO DNA binding regions within the genome.

If DNA structure is pivotal to the binding affinity of EUO, then the in vitro ChIPto-Chip analysis may not be capable of elucidating the regions of the chlamydial genome that EUO binds. This method required the sheering of genomic DNA isolated from chlamydiae to small fragments that may alter or eliminate critical conformational structure. It is known that DNA does take on different conformations throughout the developmental cycle (Solbrig, Wong et al. 1990), and investigators have shown that DNA structure does play a role in transcription (Barry, Brickman et al. 1993; Niehus, Cheng et al. 2008; Case, Peterson et al. 2010). It has been noted that EUO introduces bends in DNA (Zhang, Douglas et al. 1998), but it is not known if its binding requires DNA to be in a particular structure.

\section{In Vivo Binding of EUO}

Thirty genes were identified in my in vivo study. Although no clear commonality could be discerned among the genes, a discussion of a few of them is worthwhile. The putative thiol:disulfide exchange protein, although not experimentally examined in Chlamydia, causes the oxidation and the reduction of a disulfide bonds (Holmgren 1989). The outer membranes of EBs are extensively cross-linked, but upon entering into their host cell, the EBs convert to RBs in which outer membranes are not cross-linked. EUO is seen to be expressed early in the developmental cycle and absent in EBs. From the ChIPto-Chip data, it can be speculated that the expression of EUO may play a role in the expression of the thiol:disulfide gene, thus causing the reduction of the outer membrane.

$\operatorname{clp} B$ is another gene that may be regulated by EUO. $\operatorname{clp} B$, in other species, functions as a molecular chaperone that assists with the folding, assembly, and translocation of other proteins during high-temperature stress (Eriksson and Clarke 1996; Eriksson and Clarke 2000; Hong and Vierling 2000). Increase in temperature results in chlamydiae entering into a persistent state (Kahane and Friedman 1992). EUO transcripts are upregulated during an IFN- $\gamma$ induced persistence (Ouellette, Hatch et al. 2006) and may also be upregulated during heat-induced persistence, although this has not been verified. It is possible that the increase in production of EUO can result in increased regulation of $\operatorname{clp} B$, facilitating the ability for chlamydia to remain in a persistent state. 
$r s b V$ is a member of the partner switching mechanism that has been seen in other bacteria to help regulate the activity of the sigma factor, sigma B (Dufour and Haldenwang 1994). Chlamydia spp. encode for two RsbV proteins designated as RsbV1 and RsbV2 and both encoding genes are expressed throughout the developmental cycle (Douglas and Hatch 2000). The regulatory function of the two RsbV proteins has not been elucidated although it has been noted that both RsbV1 and RsbV2 are capable of binding the anti-sigma factor RsbW (Hua, Hefty et al. 2006). EUO was seen to bind the regulatory gene $r s b V 1$ and not bind $r s b V 2$. Binding only one of the rsbV proteins may serve as a way to regulate the possible redundancy in function of these two similar proteins.

\section{EUO AS A POSSIBLE RNA CHAPERONE}

My studies examined the binding of EUO to DNA. It is possible that EUO regulates genetic expression through binding to RNA. Host factor Q $\beta$ (Hfq) is a DNA binding protein although its major role is to bind small regulatory RNA and facilitate their antisense interaction with its target. Hfq was initially discovered as an essential factor for the replication of bacteriophage $\mathrm{Q} \beta$ in E. coli (August, Eoyang et al. 1970). Since then, it has been discovered in Vibrio cholerae, Salmonella typhimurium, Pseudomonas aeuroginosa, Staphlycoccus aureus. Hfq is essential for translation for the sigma factor RpoS in S. typhimurium (Brown and Elliott 1996). It also has been shown in $V$. cholerae to be responsible for aiding the degradation of micX transcript in an RNase Edependent fashion (Davis and Waldor 2007). Small regulatory RNAs have been described in Chlamydia (Abdelrahman, Rose et al. 2011), but Hfq is not encoded by chlamydial genomes. Could EUO be an RNA binding protein with Hfq-like function? If true, this would explain the promiscuous DNA binding nature of EUO, since RNA binding proteins can also bind to DNA. The studies of Zhang et al. (2000) concluded that EUO does not bind to RNA, but this possibility needs to be re-examined more carefully. 


\section{LIST OF REFERENCES}

Abdelrahman, Y. M., L. A. Rose, et al. (2010). "Developmental expression of non-coding RNAs in Chlamydia trachomatis during normal and persistent growth." Nucleic Acids Res 39(5): 1843-1854.

Aldous, M. B., J. T. Grayston, et al. (1992). "Seroepidemiology of Chlamydia pneumoniae TWAR infection in Seattle families, 1966-1979." J Infect Dis 166(3): 646-649.

Altuvia, S., A. Zhang, et al. (1998). "The Escherichia coli OxyS regulatory RNA represses fhlA translation by blocking ribosome binding." EMBO J 17(20): 60696075 .

Andersen, A. A. and D. Vanrompay (2000). "Avian chlamydiosis." Rev Sci Tech 19(2): 396-404.

August, J. T., L. Eoyang, et al. (1970). "Phage-specific and host proteins in the replication of bacteriophage RNA." Fed Proc 29(3): 1170-1175.

Bannantine, J. P., R. S. Griffiths, et al. (2000). "A secondary structure motif predictive of protein localization to the chlamydial inclusion membrane." Cell Microbiol 2(1): $35-47$.

Bannantine, J. P., D. D. Rockey, et al. (1998). "Tandem genes of Chlamydia psittaci that encode proteins localized to the inclusion membrane." Mol Microbiol 28(5): 1017-1026.

Barrios, H., B. Valderrama, et al. (1999). "Compilation and analysis of sigma(54)dependent promoter sequences." Nucleic Acids Res 27(22): 4305-4313.

Barry, C. E., 3rd, T. J. Brickman, et al. (1993). "Hc1-mediated effects on DNA structure: a potential regulator of chlamydial development." Mol Microbiol 9(2): 273-283.

Belland, R. J., D. E. Nelson, et al. (2003). "Transcriptome analysis of chlamydial growth during IFN-gamma-mediated persistence and reactivation." Proc Natl Acad Sci U S A 100(26): 15971-15976.

Belland, R. J., G. Zhong, et al. (2003). "Genomic transcriptional profiling of the developmental cycle of Chlamydia trachomatis." Proc Natl Acad Sci U S A 100(14): 8478-8483.

Benson, A. K. and W. G. Haldenwang (1993). "Bacillus subtilis sigma B is regulated by a binding protein (RsbW) that blocks its association with core RNA polymerase." Proc Natl Acad Sci U S A 90(6): 2330-2334. 
Brabetz, W. and H. Brade (1997). "Molecular cloning, sequence analysis and functional characterization of the gene kdsA, encoding 3-deoxy-D-manno-2-octulosonate-8phosphate synthase of Chlamydia psittaci 6BC." Eur J Biochem 244(1): 66-73.

Brickman, T. J., C. E. Barry, 3rd, et al. (1993). "Molecular cloning and expression of hctB encoding a strain-variant chlamydial histone-like protein with DNA-binding activity." J Bacteriol 175(14): 4274-4281.

Brown, K. L. and K. T. Hughes (1995). "The role of anti-sigma factors in gene regulation." Mol Microbiol 16(3): 397-404.

Brown, L. and T. Elliott (1996). "Efficient translation of the RpoS sigma factor in Salmonella typhimurium requires host factor I, an RNA-binding protein encoded by the hfq gene." J Bacteriol 178(13): 3763-3770.

Bush, R. M. and K. D. Everett (2001). "Molecular evolution of the Chlamydiaceae." Int J Syst Evol Microbiol 51(13): 203-220.

Campbell, S., S. J. Richmond, et al. (1989). "The development of Chlamydia trachomatis inclusions within the host eukaryotic cell during interphase and mitosis." J Gen Microbiol 135(5): 1153-1165.

Case, E. D., E. M. Peterson, et al. (2010). "Promoters for Chlamydia type III secretion genes show a differential response to DNA supercoiling that correlates with temporal expression pattern." J Bacteriol 192(10): 2569-2574.

Chen, C., D. Chen, et al. (2006). "The hypothetical protein CT813 is localized in the Chlamydia trachomatis inclusion membrane and is immunogenic in women urogenitally infected with C. trachomatis." Infect Immun 74(8): 4826-4840.

Chen, J. C. and R. S. Stephens (1997). "Chlamydia trachomatis glycosaminoglycandependent and independent attachment to eukaryotic cells." Microb Pathog 22(1): 23-30.

Clausen, J. D., G. Christiansen, et al. (1997). "Chlamydia trachomatis utilizes the host cell microtubule network during early events of infection." Mol Microbiol 25(3): 441-449.

Clifton, D. R., C. A. Dooley, et al. (2005). "Tyrosine phosphorylation of the chlamydial effector protein Tarp is species specific and not required for recruitment of actin." Infect Immun 73(7): 3860-3868.

Clifton, D. R., K. A. Fields, et al. (2004). "A chlamydial type III translocated protein is tyrosine-phosphorylated at the site of entry and associated with recruitment of actin." Proc Natl Acad Sci U S A 101(27): 10166-10171. 
Cocchiaro, J. L., Y. Kumar, et al. (2008). "Cytoplasmic lipid droplets are translocated into the lumen of the Chlamydia trachomatis parasitophorous vacuole." Proc Natl Acad Sci U S A 105(27): 9379-9384.

Conant, C. G. and R. S. Stephens (2007). "Chlamydia attachment to mammalian cells requires protein disulfide isomerase." Cell Microbiol 9(1): 222-232.

Costerton, J. W., L. Poffenroth, et al. (1976). "Ultrastructural studies of the nucleoids of the pleomorphic forms of Chlamydia psittaci 6BC: a comparison with bacteria." Can J Microbiol 22(1): 16-28.

Crenshaw, R. W., M. J. Fahr, et al. (1990). "Developmental cycle-specific host-free RNA synthesis in Chlamydia spp." Infect Immun 58(10): 3194-3201.

Davis, B. M. and M. K. Waldor (2007). "RNase E-dependent processing stabilizes MicX, a Vibrio cholerae sRNA." Mol Microbiol 65(2): 373-385.

Davis, C. H., J. E. Raulston, et al. (2002). "Protein disulfide isomerase, a component of the estrogen receptor complex, is associated with Chlamydia trachomatis serovar E attached to human endometrial epithelial cells." Infect Immun 70(7): 34133418 .

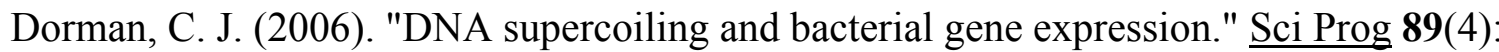
$151-166$.

Douglas, A. L. and T. P. Hatch (1995). "Functional analysis of the major outer membrane protein gene promoters of Chlamydia trachomatis." J Bacteriol 177(21): 62866289.

Douglas, A. L. and T. P. Hatch (2000). "Expression of the transcripts of the sigma factors and putative sigma factor regulators of Chlamydia trachomatis L2." Gene 247(2): 209-214.

Douglas, A. L., N. K. Saxena, et al. (1994). "Enhancement of in vitro transcription by addition of cloned, overexpressed major sigma factor of Chlamydia psittaci 6BC." J Bacteriol 176(10): 3033-3039.

Dufour, A. and W. G. Haldenwang (1994). "Interactions between a Bacillus subtilis antisigma factor (RsbW) and its antagonist (RsbV)." J Bacteriol 176(7): 1813-1820.

Eriksson, M. J. and A. K. Clarke (1996). "The heat shock protein ClpB mediates the development of thermotolerance in the cyanobacterium Synechococcus sp. strain PCC 7942." J Bacteriol 178(16): 4839-4846.

Eriksson, M. J. and A. K. Clarke (2000). "The Escherichia coli heat shock protein ClpB restores acquired thermotolerance to a cyanobacterial clpB deletion mutant." Cell Stress Chaperones 5(3): 255-264. 
Everett, K. D., R. M. Bush, et al. (1999). "Emended description of the order Chlamydiales, proposal of Parachlamydiaceae fam. nov. and Simkaniaceae fam. nov., each containing one monotypic genus, revised taxonomy of the family Chlamydiaceae, including a new genus and five new species, and standards for the identification of organisms." Int J Syst Bacteriol 49(2): 415-440.

Everett, K. D. and T. P. Hatch (1995). "Architecture of the cell envelope of Chlamydia psittaci 6BC." J Bacteriol 177(4): 877-882.

Fadel, S. and A. Eley (2008). "Differential glycosaminoglycan binding of Chlamydia trachomatis OmcB protein from serovars E and LGV." J Med Microbiol 57(9): 1058-1061.

Fahr, M. J., A. L. Douglas, et al. (1995). "Characterization of late gene promoters of Chlamydia trachomatis." J Bacteriol 177(15): 4252-4260.

Fahr, M. J., K. S. Sriprakash, et al. (1992). "Convergent and overlapping transcripts of the Chlamydia trachomatis 7.5-kb plasmid." Plasmid 28(3): 247-257.

Friis, R. R. (1972). "Interaction of L cells and Chlamydia psittaci: entry of the parasite and host responses to its development." J Bacteriol 110(2): 706-721.

Gerard, H. C., J. A. Whittum-Hudson, et al. (2006). "Synovial Chlamydia trachomatis up regulates expression of a panel of genes similar to that transcribed by Mycobacterium tuberculosis during persistent infection." Ann Rheum Dis 65(3): 321-327.

Gohler, T., S. Jager, et al. (2005). "Mutant p53 proteins bind DNA in a DNA structureselective mode." Nucleic Acids Res 33(3): 1087-1100.

Gottesman, S., G. Storz, et al. (2001). "Small RNA regulators of translation: mechanisms of action and approaches for identifying new small RNAs." Cold Spring Harb Symp Quant Biol 66: 353-362.

Grayston, J. T. (1992). "Infections caused by Chlamydia pneumoniae strain TWAR." Clin Infect Dis 15(5): 757-761.

Grieshaber, N. A., S. S. Grieshaber, et al. (2006). "A small RNA inhibits translation of the histone-like protein $\mathrm{Hcl}$ in Chlamydia trachomatis." Mol Microbiol 59(2): 541-550.

Grieshaber, S. S., N. A. Grieshaber, et al. (2003). "Chlamydia trachomatis uses host cell dynein to traffic to the microtubule-organizing center in a p50 dynamitinindependent process." J Cell Sci 116(18): 3793-3802.

Gruber, T. M. and C. A. Gross (2003). "Multiple sigma subunits and the partitioning of bacterial transcription space." Annu Rev Microbiol 57: 441-466. 
Hackstadt, T. (1986). "Identification and properties of chlamydial polypeptides that bind eucaryotic cell surface components." J Bacteriol 165(1): 13-20.

Hackstadt, T., W. Baehr, et al. (1991). "Chlamydia trachomatis developmentally regulated protein is homologous to eukaryotic histone H1." Proc Natl Acad Sci U

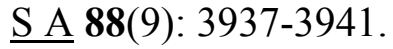

Hackstadt, T., M. A. Scidmore, et al. (1995). "Lipid metabolism in Chlamydia trachomatis-infected cells: directed trafficking of Golgi-derived sphingolipids to the chlamydial inclusion." Proc Natl Acad Sci U S A 92(11): 4877-4881.

Hahn, D. L., D. Bukstein, et al. (1998). "Evidence for Chlamydia pneumoniae infection in steroid-dependent asthma." Ann Allergy Asthma Immunol 80(1): 45-49.

Hatch, T. P. (1975). "Utilization of L-cell nucleoside triphosphates by Chlamydia psittaci for ribonucleic acid synthesis." J Bacteriol 122(2): 393-400.

Hatch, T. P. (1976). "Utilization of exogenous thymidine by Chlamydia psittaci growing in the thymidine kinase-containing and thymidine kinase-deficient L cells." $\underline{\mathrm{J}}$ Bacteriol 125(2): 706-712.

Hatch, T. P., E. Al-Hossainy, et al. (1982). "Adenine nucleotide and lysine transport in Chlamydia psittaci." J Bacteriol 150(2): 662-670.

Hatch, T. P., I. Allan, et al. (1984). "Structural and polypeptide differences between envelopes of infective and reproductive life cycle forms of Chlamydia spp." $\underline{\mathrm{J}}$ Bacteriol 157(1): 13-20.

Hatch, T. P., M. Miceli, et al. (1986). "Synthesis of disulfide-bonded outer membrane proteins during the developmental cycle of Chlamydia psittaci and Chlamydia trachomatis." J Bacteriol 165(2): 379-385.

Hefty, P. S. and R. S. Stephens (2007). "Chlamydial type III secretion system is encoded on ten operons preceded by sigma 70-like promoter elements." J Bacteriol 189(1): 198-206.

Helmann, J. D. (1991). "Alternative sigma factors and the regulation of flagellar gene expression." Mol Microbiol 5(12): 2875-2882.

Helmann, J. D. (2002). "The extracytoplasmic function (ECF) sigma factors." Adv Microb Physiol 46: 47-110.

Hodinka, R. L. and P. B. Wyrick (1986). "Ultrastructural study of mode of entry of Chlamydia psittaci into L-929 cells." Infect Immun 54(3): 855-863.

Holmgren, A. (1989). "Thioredoxin and glutaredoxin systems." J Biol Chem 264(24): 13963-13966. 
Hong, S. W. and E. Vierling (2000). "Mutants of Arabidopsis thaliana defective in the acquisition of tolerance to high temperature stress." Proc Natl Acad Sci U S A 97(8): 4392-4397.

Hoover, T. R., E. Santero, et al. (1990). "The integration host factor stimulates interaction of RNA polymerase with NIFA, the transcriptional activator for nitrogen fixation operons." Cell 63(1): 11-22.

Hsia, R. C., Y. Pannekoek, et al. (1997). "Type III secretion genes identify a putative virulence locus of Chlamydia." Mol Microbiol 25(2): 351-359.

Hua, L., P. S. Hefty, et al. (2006). "Core of the partner switching signalling mechanism is conserved in the obligate intracellular pathogen Chlamydia trachomatis." Mol Microbiol 59(2): 623-636.

Huang, J., C. F. Lesser, et al. (2008). "The essential role of the CopN protein in Chlamydia pneumoniae intracellular growth." Nature 456(7218): 112-115.

Hybiske, K. and R. S. Stephens (2007). "Mechanisms of Chlamydia trachomatis entry into nonphagocytic cells." Infect Immun 75(8): 3925-3934.

Hybiske, K. and R. S. Stephens (2007). "Mechanisms of host cell exit by the intracellular bacterium Chlamydia." Proc Natl Acad Sci U S A 104(27): 11430-11435.

Kahane, S. and M. G. Friedman (1992). "Reversibility of heat shock in Chlamydia trachomatis." FEMS Microbiol Lett 76(1): 25-30.

Kalman, S., W. Mitchell, et al. (1999). "Comparative genomes of Chlamydia pneumoniae and C. trachomatis." Nat Genet 21(4): 385-389.

Kang, C. M., M. S. Brody, et al. (1996). "Homologous pairs of regulatory proteins control activity of Bacillus subtilis transcription factor sigma(b) in response to environmental stress." J Bacteriol 178(13): 3846-3853.

Karlinsey, J. E. and K. T. Hughes (2006). "Genetic transplantation: Salmonella enterica serovar Typhimurium as a host to study sigma factor and anti-sigma factor interactions in genetically intractable systems." J Bacteriol 188(1): 103-114.

Kaul, R., A. Hoang, et al. (1997). "The chlamydial EUO gene encodes a histone H1specific protease." J Bacteriol 179(18): 5928-5934.

Kim, E., N. Albrechtsen, et al. (1997). "DNA-conformation is an important determinant of sequence-specific DNA binding by tumor suppressor p53." Oncogene 15(7): 857-869.

Kivi, M., F. D. Koedijk, et al. (2008). "Evaluation prompting transition from enhanced to routine surveillance of lymphogranuloma venereum (LGV) in the Netherlands." Euro Surveill 13(14): 8087 
Koehler, J. E., R. R. Burgess, et al. (1990). "Chlamydia trachomatis RNA polymerase major sigma subunit. Sequence and structural comparison of conserved and unique regions with Escherichia coli sigma 70 and Bacillus subtilis sigma 43." $\underline{\mathrm{J}}$ Biol Chem 265(22): 13206-13214.

Kozak, N. A., S. Mattoo, et al. (2005). "Interactions between partner switcher orthologs BtrW and BtrV regulate type III secretion in Bordetella." J Bacteriol 187(16): 5665-5676.

Kuo, C. C. and T. Grayston (1976). "Interaction of Chlamydia trachomatis organisms and HeLa 229 cells." Infect Immun 13(4): 1103-1109.

Kustu, S., E. Santero, et al. (1989). "Expression of sigma 54 (ntrA)-dependent genes is probably united by a common mechanism." Microbiol Rev 53(3): 367-376.

Luo, J., T. Jia, et al. (2007). "Hypothetical protein Cpn0308 is localized in the Chlamydia pneumoniae inclusion membrane." Infect Immun 75(1): 497-503.

Majeed, M., J. D. Ernst, et al. (1994). "Selective translocation of annexins during intracellular redistribution of Chlamydia trachomatis in HeLa and McCoy cells." Infect Immun 62(1): 126-134.

Majeed, M. and E. Kihlstrom (1991). "Mobilization of F-actin and clathrin during redistribution of Chlamydia trachomatis to an intracellular site in eucaryotic cells." Infect Immun 59(12): 4465-4472.

Marques, M. V., S. L. Gomes, et al. (1997). "A gene coding for a putative sigma 54 activator is developmentally regulated in Caulobacter crescentus." J Bacteriol 179(17): 5502-5510.

Mathews, S. A. and K. S. Sriprakash (1994). "The RNA polymerase of Chlamydia trachomatis has a flexible sequence requirement at the -10 and -35 boxes of its promoters." J Bacteriol 176(12): 3785-3789.

Mathews, S. A. and P. Timms (2000). "Identification and mapping of sigma-54 promoters in Chlamydia trachomatis." J Bacteriol 182(21): 6239-6242.

Matsumoto, A. (1982). "Surface projections of Chlamydia psittaci elementary bodies as revealed by freeze-deep-etching." J Bacteriol 151(2): 1040-1042.

Mattoo, S., M. H. Yuk, et al. (2004). "Regulation of type III secretion in Bordetella." Microbiol 52(4): 1201-1214.

McCleary, W. R. and D. R. Zusman (1990). "FrzE of Myxococcus xanthus is homologous to both CheA and CheY of Salmonella typhimurium." Proc Natl Acad Sci U S A 87(15): 5898-5902. 
Mirel, D. B., V. M. Lustre, et al. (1992). "An operon of Bacillus subtilis motility genes transcribed by the sigma D form of RNA polymerase." J Bacteriol 174(13): 41974204.

Mital, J., N. J. Miller, et al. (2010). "Specific chlamydial inclusion membrane proteins associate with active Src family kinases in microdomains that interact with the host microtubule network." Cell Microbiol 12(9): 1235-1249.

Moelleken, K. and J. H. Hegemann (2008). "The chlamydia outer membrane protein OmcB is required for adhesion and exhibits biovar-specific differences in glycosaminoglycan binding." Mol Microbiol 67(2): 403-419.

Moorhead, A. M., J. Y. Jung, et al. (2010). "Multiple host proteins that function in phosphatidylinositol-4 phosphate metabolism are recruited to the chlamydial inclusion." Infect Immun 78(5): 1990-2007.

Moulder, J. W. (1962). "Some basic properties of the psittacosis-lymphogranuloma venereum group of agents. Structure and chemical composition of isolated particles." Ann N Y Acad Sci 98: 92-99.

Moulder, J. W. (1982). "The relation of basic biology to pathogenic potential in the genus Chlamydia." Infection 10 Suppl 1: S10-18.

Moulder, J. W. (1991). "Interaction of chlamydiae and host cells in vitro." Microbiol Rev 55(1): 143-190.

Murray, A. and M. E. Ward (1984). "Control mechanisms governing the infectivity of Chlamydia trachomatis for HeLa cells: the role of calmodulin." J Gen Microbiol 130(1): 193-201.

Neeper, I. D., D. L. Patton, et al. (1990). "Cinematographic observations of growth cycles of Chlamydia trachomatis in primary cultures of human amniotic cells." Infect Immun 58(7): 2042-2047.

Negrel, A. D. and S. P. Mariotti (1998). "WHO alliance for the global elimination of blinding trachoma and the potential use of azithromycin." Int J Antimicrob Agents 10(4): 259-262.

Nicholson, T. L., L. Olinger, et al. (2003). "Global stage-specific gene regulation during the developmental cycle of Chlamydia trachomatis." J Bacteriol 185(10): 31793189 .

Niehus, E., E. Cheng, et al. (2008). "DNA supercoiling-dependent gene regulation in Chlamydia." J Bacteriol 190(19): 6419-6427.

Norkin, L. C., S. A. Wolfrom, et al. (2001). "Association of caveolin with Chlamydia trachomatis inclusions at early and late stages of infection." Exp Cell Res 266(2): 229-238. 
Ouellette, S. P., T. P. Hatch, et al. (2006). "Global transcriptional upregulation in the absence of increased translation in Chlamydia during IFNgamma-mediated host cell tryptophan starvation." Mol Microbiol 62(5): 1387-1401.

Pagel, J. M., J. W. Winkelman, et al. (1992). "DNA topology-mediated regulation of transcription initiation from the tandem promoters of the ilvGMEDA operon of Escherichia coli." J Mol Biol 224(4): 919-935.

Perara, E., D. Ganem, et al. (1992). "A developmentally regulated chlamydial gene with apparent homology to eukaryotic histone H1." Proc Natl Acad Sci U S A 89(6): 2125-2129.

Peterson, E. M. and L. M. de la Maza (1988). "Chlamydia parasitism: ultrastructural characterization of the interaction between the chlamydial cell envelope and the host cell." J Bacteriol 170(3): 1389-1392.

Plaunt, M. R. and T. P. Hatch (1988). "Protein synthesis early in the developmental cycle of Chlamydia psittaci." Infect Immun 56(12): 3021-3025.

Pultorak, E., W. Wong, et al. (2009). "Economic burden of sexually transmitted infections: incidence and direct medical cost of Chlamydia, gonorrhea, and syphilis among Illinois adolescents and young adults, 2005-2006." Sex Transm Dis 36(10): 629-636.

Rao, X., P. Deighan, et al. (2009). "A regulator from Chlamydia trachomatis modulates the activity of RNA polymerase through direct interaction with the beta subunit and the primary sigma subunit." Genes Dev 23(15): 1818-1829.

Rau, A., S. Wyllie, et al. (2005). "Identification of Chlamydia trachomatis genomic sequences recognized by chlamydial divalent cation-dependent regulator A (DcrA)." J Bacteriol 187(2): 443-448.

Raulston, J. E. (1997). "Response of Chlamydia trachomatis serovar E to iron restriction in vitro and evidence for iron-regulated chlamydial proteins." Infect Immun 65(11): 4539-4547.

Read, T. D., G. S. Myers, et al. (2003). "Genome sequence of Chlamydophila caviae (Chlamydia psittaci GPIC): examining the role of niche-specific genes in the evolution of the Chlamydiaceae." Nucleic Acids Res 31(8): 2134-2147.

Rejman Lipinski, A., J. Heymann, et al. (2009). "Rab6 and Rab11 regulate Chlamydia trachomatis development and golgin-84-dependent Golgi fragmentation." PLoS Pathog 5(10): e1000615.

Resnikoff, S., D. Pascolini, et al. (2004). "Global data on visual impairment in the year 2002." Bull World Health Organ 82(11): 844-851. 
Rockey, D. D., E. R. Fischer, et al. (1996). "Temporal analysis of the developing Chlamydia psittaci inclusion by use of fluorescence and electron microscopy." Infect Immun 64(10): 4269-4278.

Rockey, D. D., R. A. Heinzen, et al. (1995). "Cloning and characterization of a Chlamydia psittaci gene coding for a protein localized in the inclusion membrane of infected cells." Mol Microbiol 15(4): 617-626.

Rockey, D. D. and J. L. Rosquist (1994). "Protein antigens of Chlamydia psittaci present in infected cells but not detected in the infectious elementary body." Infect Immun 62(1): 106-112.

Saikku, P., M. Leinonen, et al. (1988). "Serological evidence of an association of a novel Chlamydia, TWAR, with chronic coronary heart disease and acute myocardial infarction." Lancet 2(8618): 983-986.

Schaumburg, C. S. and M. Tan (2003). "Mutational analysis of the Chlamydia trachomatis dnaK promoter defines the optimal -35 promoter element." Nucleic Acids Res 31(2): 551-555.

Schramm, N., C. R. Bagnell, et al. (1996). "Vesicles containing Chlamydia trachomatis serovar L2 remain above pH 6 within HEC-1B cells." Infect Immun 64(4): 12081214.

Schulz, A. and W. Schumann (1996). "hrcA, the first gene of the Bacillus subtilis dnaK operon encodes a negative regulator of class I heat shock genes." J Bacteriol 178(4): 1088-1093.

Scidmore-Carlson, M. A., E. I. Shaw, et al. (1999). "Identification and characterization of a Chlamydia trachomatis early operon encoding four novel inclusion membrane proteins." Mol Microbiol 33(4): 753-765.

Scidmore, M. A., E. R. Fischer, et al. (1996). "Sphingolipids and glycoproteins are differentially trafficked to the Chlamydia trachomatis inclusion." J Cell Biol 134(2): 363-374.

Scidmore, M. A., E. R. Fischer, et al. (2003). "Restricted fusion of Chlamydia trachomatis vesicles with endocytic compartments during the initial stages of infection." Infect Immun 71(2): 973-984.

Shaw, E. I., C. A. Dooley, et al. (2000). "Three temporal classes of gene expression during the Chlamydia trachomatis developmental cycle." Mol Microbiol 37(4): 913-925.

Shen, L., Y. Shi, et al. (2000). "Identification and characterization of promoters regulating tuf expression in Chlamydia trachomatis serovar F." Arch Biochem Biophys 379(1): 46-56. 
Sheridan, S. D., C. J. Benham, et al. (1998). "Activation of gene expression by a novel DNA structural transmission mechanism that requires supercoiling-induced DNA duplex destabilization in an upstream activating sequence." J Biol Chem 273(33): 21298-21308.

Shirai, M., H. Hirakawa, et al. (2000). "Comparison of whole genome sequences of Chlamydia pneumoniae J138 from Japan and CWL029 from USA." Nucleic Acids Res 28(12): 2311-2314.

Sledjeski, D. D., A. Gupta, et al. (1996). "The small RNA, DsrA, is essential for the low temperature expression of RpoS during exponential growth in Escherichia coli." EMBO J 15(15): 3993-4000.

Solbrig, M. V., M. L. Wong, et al. (1990). "Developmental-stage-specific plasmid supercoiling in Chlamydia trachomatis." Mol Microbiol 4(9): 1535-1541.

Stamm, W. E. (1999). "Chlamydia trachomatis infections: progress and problems." $\underline{J}$ Infect Dis 179 Suppl 2: S380-383.

Stephens, R. S., S. Kalman, et al. (1998). "Genome sequence of an obligate intracellular pathogen of humans: Chlamydia trachomatis." Science 282(5389): 754-759.

Stephens, R. S., C. C. Kuo, et al. (1985). "Molecular cloning and expression of Chlamydia trachomatis major outer membrane protein antigens in Escherichia coli." Infect Immun 47(3): 713-718.

Stephens, R. S., G. Myers, et al. (2009). "Divergence without difference: phylogenetics and taxonomy of Chlamydia resolved." FEMS Immunol Med Microbiol 55(2): 115-119.

Stephens, R. S., E. A. Wagar, et al. (1988). "Developmental regulation of tandem promoters for the major outer membrane protein gene of Chlamydia trachomatis." J Bacteriol 170(2): 744-750.

Stuart, E. S., W. C. Webley, et al. (2003). "Lipid rafts, caveolae, caveolin-1, and entry by Chlamydiae into host cells." Exp Cell Res 287(1): 67-78.

Su, H. and H. D. Caldwell (1998). "Sulfated polysaccharides and a synthetic sulfated polymer are potent inhibitors of Chlamydia trachomatis infectivity in vitro but lack protective efficacy in an in vivo murine model of chlamydial genital tract infection." Infect Immun 66(3): 1258-1260.

Su, H., Y. X. Zhang, et al. (1988). "Differential effect of trypsin on infectivity of Chlamydia trachomatis: loss of infectivity requires cleavage of major outer membrane protein variable domains II and IV." Infect Immun 56(8): 2094-2100. 
Tamura, A., A. Matsumoto, et al. (1971). "Electron microscopic observations on the structure of the envelopes of mature elementary bodies and developmental reticulate forms of Chlamydia psittaci." J Bacteriol 105(1): 355-360.

Tan, M. and J. N. Engel (1996). "Identification of sequences necessary for transcription in vitro from the Chlamydia trachomatis rRNA P1 promoter." J Bacteriol 178(23): 6975-6982.

Tan, M., B. Wong, et al. (1996). "Transcriptional organization and regulation of the dnaK and groE operons of Chlamydia trachomatis." J Bacteriol 178(23): 6983-6990.

Taraktchoglou, M., A. A. Pacey, et al. (2001). "Infectivity of Chlamydia trachomatis serovar LGV but not $\mathrm{E}$ is dependent on host cell heparan sulfate." Infect Immun 69(2): 968-976.

Tipples, G. and G. McClarty (1993). "The obligate intracellular bacterium Chlamydia trachomatis is auxotrophic for three of the four ribonucleoside triphosphates." Mol Microbiol 8(6): 1105-1114.

Tjaden, J., H. H. Winkler, et al. (1999). "Two nucleotide transport proteins in Chlamydia trachomatis, one for net nucleoside triphosphate uptake and the other for transport of energy." J Bacteriol 181(4): 1196-1202.

Todd, W. J. and H. D. Caldwell (1985). "The interaction of Chlamydia trachomatis with host cells: ultrastructural studies of the mechanism of release of a biovar II strain from HeLa 229 cells." J Infect Dis 151(6): 1037-1044.

Tribby, I. and J. W. Moulder (1966). "Availability of bases and nucleosides as precursors of nucleic acids in L cells and in the agent of meningopneumonitis." J Bacteriol 91(6): 2362-2367.

Wagar, E. A. and R. S. Stephens (1988). "Developmental-form-specific DNA-binding proteins in Chlamydia spp." Infect Immun 56(7): 1678-1684.

Wagels, G., S. Rasmussen, et al. (1994). "Comparison of Chlamydia pneumoniae isolates by western blot (immunoblot) analysis and DNA sequencing of the omp 2 gene." J Clin Microbiol 32(11): 2820-2823.

Wang, J., L. Chen, et al. (2009). "A chlamydial type III-secreted effector protein (Tarp) is predominantly recognized by antibodies from humans infected with Chlamydia trachomatis and induces protective immunity against upper genital tract pathologies in mice." Vaccine 27(22): 2967-2980.

Wang, S. P. and J. T. Grayston (1991). "Three new serovars of Chlamydia trachomatis: Da, Ia, and L2a." J Infect Dis 163(2): 403-405.

Wang, S. P., C. C. Kuo, et al. (1985). "Immunotyping of Chlamydia trachomatis with monoclonal antibodies." J Infect Dis 152(4): 791-800. 
Wassarman, D. A. and J. A. Steitz (1992). "Interactions of small nuclear RNA's with precursor messenger RNA during in vitro splicing." Science 257(5078): 19181925.

Watson, M. W., P. R. Lambden, et al. (1994). "Immunoreactivity of the $60 \mathrm{kDa}$ cysteinerich proteins of Chlamydia trachomatis, Chlamydia psittaci and Chlamydia pneumoniae expressed in Escherichia coli." Microbiology 140(8): 2003-2011.

Webley, W. C., L. C. Norkin, et al. (2004). "Caveolin-2 associates with intracellular chlamydial inclusions independently of caveolin-1." BMC Infect Dis 4: 23.

Wehrl, W., V. Brinkmann, et al. (2004). "From the inside out--processing of the Chlamydial autotransporter PmpD and its role in bacterial adhesion and activation of human host cells." Mol Microbiol 51(2): 319-334.

Wichlan, D. G. and T. P. Hatch (1993). "Identification of an early-stage gene of Chlamydia psittaci 6BC." J Bacteriol 175(10): 2936-2942.

Wilson, A. C. and M. Tan (2002). "Functional analysis of the heat shock regulator HrcA of Chlamydia trachomatis." J Bacteriol 184(23): 6566-6571.

Wilson, A. C. and M. Tan (2004). "Stress response gene regulation in Chlamydia is dependent on HrcA-CIRCE interactions." J Bacteriol 186(11): 3384-3391.

Wylie, J. L., G. M. Hatch, et al. (1997). "Host cell phospholipids are trafficked to and then modified by Chlamydia trachomatis." J Bacteriol 179(23): 7233-7242.

Wyllie, S. and J. E. Raulston (2001). "Identifying regulators of transcription in an obligate intracellular pathogen: a metal-dependent repressor in Chlamydia trachomatis." Mol Microbiol 40(4): 1027-1036.

Wyrick, P. B., J. Choong, et al. (1989). "Entry of genital Chlamydia trachomatis into polarized human epithelial cells." Infect Immun 57(8): 2378-2389.

Yu, H. H., E. G. Di Russo, et al. (2006). "Mutational analysis of the promoter recognized by Chlamydia and Escherichia coli sigma(28) RNA polymerase." J Bacteriol 188(15): 5524-5531.

Yu, H. H., D. Kibler, et al. (2006). "In silico prediction and functional validation of sigma28-regulated genes in Chlamydia and Escherichia coli." J Bacteriol 188(23): 8206-8212.

Yu, H. H. and M. Tan (2003). "Sigma28 RNA polymerase regulates hetB, a late developmental gene in Chlamydia." Mol Microbiol 50(2): 577-584.

Zhang, J. P. and R. S. Stephens (1992). "Mechanism of C. trachomatis attachment to eukaryotic host cells." Cell 69(5): 861-869. 
Zhang, L., A. L. Douglas, et al. (1998). "Characterization of a Chlamydia psittaci DNA binding protein (EUO) synthesized during the early and middle phases of the developmental cycle." Infect Immun 66(3): 1167-1173.

Zhang, L., M. M. Howe, et al. (2000). "Characterization of in vitro DNA binding sites of the EUO protein of Chlamydia psittaci." Infect Immun 68(3): 1337-1349.

Zhong, J., A. L. Douglas, et al. (2001). "Characterization of integration host factor (IHF) binding upstream of the cysteine-rich protein operon (omcAB) promoter of Chlamydia trachomatis LGV serovar L2." Mol Microbiol 41(2): 451-462. 


\section{VITA}

Cory L. Blackwell was born in Beaumont, Mississippi in 1979. He attended Beaumont Elementary school and graduated from Perry Central High School in New Augusta in May of 1997. The following August he attended and graduated from Dillard University in May 2001 with a Bachelor of Science degree in Biology (cum laude).

Following graduation he began work at Coca-Cola Enterprises as a Quality Assurance Technician in New Orleans, Louisiana. In August 2003, he entered the Molecular Sciences program at the University of Tennessee Health Science Center. He completed his Doctor of Philosophy in Molecular Science in 2011. 